\title{
Comparing and counting logs in direct and effective methods of $Q C D$ resummation
}

\author{
Leandro G. Almeida, ${ }^{a, b}$ Stephen D. Ellis, ${ }^{c}$ Christopher Lee, ${ }^{d}$ George Sterman, ${ }^{e}$ \\ Ilmo Sung ${ }^{f, g}$ and Jonathan R. Walsh ${ }^{h, i}$ \\ ${ }^{a}$ Laboratoire de Physique Théorique, Université Paris-Sud 11 and CNRS, \\ 91405 Orsay Cedex, France \\ ${ }^{b}$ Institut de Biologie de l'École Normale Supérieure (IBENS), \\ Inserm 1024-CNRS 8197, 46 rue d'Ulm, 75005 Paris, France ${ }^{1}$ \\ ${ }^{c}$ Department of Physics, University of Washington, \\ Seattle, WA 98195, U.S.A. \\ ${ }^{d}$ Theoretical Division, MS B283, Los Alamos National Laboratory, \\ Los Alamos, NM 87544, U.S.A. \\ ${ }^{e}$ C.N. Yang Institute for Theoretical Physics, Stony Brook University, \\ Stony Brook, NY 11794, U.S.A. \\ ${ }^{f}$ Department of Applied Physics, New York University, \\ Brooklyn, NY 11201, U.S.A. \\ ${ }^{g}$ Queens College, City University of New York, \\ Flushing, NY 1136r, U.S.A. \\ ${ }^{h}$ Lawrence Berkeley National Laboratory, University of California, \\ Berkeley, CA 94720, U.S.A. \\ ${ }^{i}$ Berkeley Center for Theoretical Physics, University of California, \\ Berkeley, CA 94720, U.S.A. \\ E-mail: leandro.g.almeida@gmail.com, sdellis@uw.edu, clee@lanl.gov, \\ george.sterman@stonybrook.edu, ilmo.sung@nyu.edu, jwalsh@lbl.gov
}

ABSTRACT: We compare methods to resum logarithms in event shape distributions as they have been used in perturbative QCD directly and in effective field theory. We demonstrate that they are equivalent. In showing this equivalence, we are able to put standard softcollinear effective theory (SCET) formulae for cross sections in momentum space into a novel form more directly comparable with standard QCD formulae, and endow the QCD formulae with dependence on separated hard, jet, and soft scales, providing potential ways to improve estimates of theoretical uncertainty. We show how to compute cross sections in momentum space to keep them as accurate as the corresponding expressions in Laplace

\footnotetext{
${ }^{1}$ Current address.
} 
space. In particular, we point out that that care is required in truncating differential distributions at $\mathrm{N}^{k} \mathrm{LL}$ accuracy to ensure they match the accuracy of the corresponding cumulant or Laplace transform. We explain how to avoid such mismatches at $\mathrm{N}^{k} \mathrm{LL}$ accuracy, and observe why they can also be avoided by working to $\mathrm{N}^{k} \mathrm{LL}^{\prime}$ accuracy.

KEYwORDS: Resummation, QCD

ARXIV EPRINT: 1401.4460 


\section{Contents}

1 Introduction $\quad 2$

2 Orders of resummed perturbative accuracy 5

2.1 Definition of $\mathrm{N}^{k} \mathrm{LL}$ accuracy: counting in the Laplace exponent 5

2.2 Original CTTW convention: counting in the cumulant 8

3 Comparison of direct QCD and SCET resummation 10

$\begin{array}{lll}3.1 & \text { Factorization and resummation of event shape distributions } & 11\end{array}$

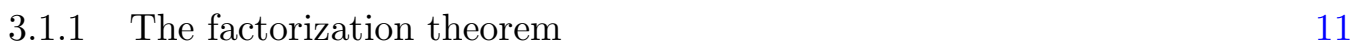

$\begin{array}{lll}\text { 3.1.2 Jet and soft functions in direct QCD } & 12\end{array}$

$\begin{array}{lll}\text { 3.1.3 Jet and soft functions in SCET } & 14\end{array}$

$\begin{array}{lll}3.2 & \text { Resummed event shape distributions in SCET } & 15\end{array}$

3.2.1 Perturbative expansions and evolution of hard, jet, and soft functions 15

$\begin{array}{lll}3.2 .2 & \text { Evolution in SCET } & 18\end{array}$

3.2.3 Resummed cross section in SCET 20

3.3 Resummed event shape distributions in QCD 22

3.4 Equivalence between QCD and SCET resummation 25

$\begin{array}{lll}3.5 & \text { Interlude } & 30\end{array}$

4 Achieving $\mathrm{N}^{k}$ LL logarithmic accuracy $\quad 32$

4.1 Laplace transform 32

$\begin{array}{lll}4.2 & \text { Cumulant } & 39\end{array}$

4.2.1 Resummed cumulant in SCET formalism 39

4.2.2 Computing Laplace transform and cumulant to consistent accuracy 40

$\begin{array}{lll}4.3 & \text { Distribution } & 48\end{array}$

4.3.1 Keeping cumulant and distribution to consistent accuracy $\quad 50$

4.3.2 Counting accuracy of cumulant and distribution in dQCD formalism 54

5 Final formulae and prescriptions for $\mathrm{N}^{k} \mathrm{LL}\left({ }^{\prime}\right)$ accuracy $\quad 55$

$\begin{array}{ll}5.1 \text { Laplace transform } & 56\end{array}$

$\begin{array}{lll}5.2 \text { Cumulant } & 57\end{array}$

$\begin{array}{lll}5.3 & \text { Differential distribution } & 59\end{array}$

6 Numerical comparison of angularity distributions 60

$\begin{array}{lll}7 & \text { Conclusions } & 63\end{array}$

$\begin{array}{ll}\text { A Plus distributions } & 66\end{array}$

$\begin{array}{ll}\text { B Laplace transforms } & 67\end{array}$

$\begin{array}{lll}\text { C Anomalous dimensions } & 68\end{array}$ 


\section{Introduction}

The theory Quantum Chromodynamics (QCD) is remarkably successful in describing the strong interaction. Thanks to the phenomenon of asymptotic freedom $[1,2]$, many inclusive strong interaction cross sections can be predicted to excellent accuracy in perturbation theory, expanding order by order in the strong coupling $\alpha_{s}(\mu)$.

Cross sections that are not fully inclusive over final-state hadrons, however, require careful treatment in perturbation theory due to the appearance of large logarithms $\ln ^{k}\left(\mu_{1} / \mu_{2}\right)$ at each order in fixed-order perturbation theory. The logarithms are of ratios of scales $\mu_{1,2}$ that are used to define the exclusivity of the measurement. If the scales are widely separated, the logs are large, and the expansion in $\alpha_{s}$ is poorly behaved. In particular, such logs appear in cross sections that count hadronic jets [3]. They may depend on ratios of energy cuts $\Lambda$ on the final state to the total interaction energy $Q$. The logs may also depend on masses of jets that are measured in the final state. Methods to resum these logarithms to all orders in $\alpha_{s}$ may or may not yet exist, depending on the type of measurement.

Event shapes provide a large class of observables that can be used to measure the jet-like structure of the final state in collisions producing hadrons [4]. We consider those event shapes that are "global" observables, which sum over all the final-state hadrons with a single weight measure $e$ that introduces sensitivity of the cross section to a single collinear scale and a single soft scale. Non-global observables which induce sensitivity to multiple soft scales will not be considered here [5,6]. For simplicity we consider event shapes in leptonic collisions $e^{+} e^{-} \rightarrow X$ producing hadrons $X$. Our observations and conclusions apply more generally to the resummation of logs in other types of collisions and measurements as well. A very familiar event shape is the thrust $\tau=1-T[7]$, defined here by

$$
\tau \equiv 1-T=1-\frac{1}{Q} \max _{\hat{\mathbf{t}}} \sum_{i \in X}\left|\hat{\mathbf{t}} \cdot \mathbf{p}_{i}\right|,
$$

where $Q$ is the center-of-mass collision energy, $X$ the final hadronic state, $\mathbf{p}_{i}$ the 3 momentum of particle $i$, and $\hat{\mathbf{t}}$ the thrust axis, defined as the axis that maximizes the sum over $i$ (and thus minimizes $\tau$ ) in eq. (1.1). The thrust is one of a continuous set of event shapes that can be written in a more generic form,

$$
e=\frac{1}{Q} \sum_{i \in X}\left|\mathbf{p}_{T}^{i}\right| f_{e}\left(\eta_{i}\right)
$$

where the transverse momentum $\mathbf{p}_{T}$ and rapidity $\eta$ of each particle are measured with respect to the thrust axis $\hat{\mathbf{t}}$. (Equation (1.2) applies to massless hadrons $i$ in the final state. For the generalization to massive hadrons, see $[8,9]$.) The function $f_{e}$ is taken to be continuous and to fall off sufficiently fast at large $\eta_{i}$ to preserve infrared and collinear safety $[10,11]$. In the main discussion in this paper, we will consider the class known as angularities $e=\tau_{a}[12-14]$, defined by $f_{a}(\eta)=e^{-|\eta|(1-a)}$, which are infrared safe for real numbers $a<2$, although our discussion of factorization and resummation below will be valid for $a<1$. 
Although our discussion will take place mainly within the context of angularity event shapes in $e^{+} e^{-}$collisions, our conclusions will not limited to this application. Rather they provide a concrete arena in which to draw more general lessons about different techniques to resum logs in QCD perturbation theory and the relation between them.

Measurement of an event shape such as thrust induces sensitivity of the cross section on several different energy scales. Small thrust $\tau$ means that the final state is nearly twojet-like. The cross section then depends on the hard collision energy $Q$, the typical jet mass, $Q \sqrt{\tau}$, and the energy of soft radiation from the jets, $Q \tau$. Ratios of these scales appear as $\operatorname{logs}$ of $\tau$ in the perturbative expansion of the thrust distribution. A method to resum twojet event shapes was described in [15] (CTTW) based on the soft and collinear singularities of QCD and approximations to phase space in these regions. A unified derivation of the resummation of logarithms from the factorization properties of QCD cross sections was presented in ref. [16], which are also naturally derivable using the methods of effective field theory. We summarize these methods in parallel discussions below in the context of "direct" QCD (dQCD) and soft collinear effective theory (SCET) [17-21] techniques to resum logs, applied to jet cross sections [22, 23] and event shape distributions [24].

Working directly in full QCD or going through the methods of EFT should theoretically be equivalent. The EFT, after all, is a systematic approximation to and method for computing in the full theory [25]. It is a primary goal of this paper to show that standard formulae derived in the two formalisms are, indeed, equivalent. However, some results for resummed event shapes published in the respective literature sometimes do not seem obviously equivalent and sometimes yield apparently different numerical results.

We will illustrate one such previous discrepancy and its resolution by focusing on a comparison between angularity distributions reported in [12,13] using full QCD and [26, 27] using SCET. (QCD NLL results for $\tau_{a}$ and many other event shapes are also given in [28].) In [26] a comparison of the two sets of results at next-to-leading logarithmic (NLL) accuracy was performed, and significant numerical differences were found. In this paper, we resolve the source of this discrepancy. The resolution will involve a careful consideration of the meaning of $\mathrm{N}^{k} \mathrm{LL}$ accuracy for various ways to write the cross section, e.g. as a differential distribution, the cumulative distribution (cumulant) or in Laplace or position space. We find that the results in $[12,13]$ and $[26]$ were not evaluated according to a consistent definition of NLL accuracy. Once we implement such a consistent scheme, we find that results in the two formalisms yield numerically equivalent results.

In section 2 we will review a standard definition of $\mathrm{N}^{k} \mathrm{LL}$ accuracy, namely, which terms in the exponent of the Laplace transform $\widetilde{\sigma}\left(\nu_{a}\right)$ of the event shape distribution $d \sigma / d \tau_{a}$ are accurately predicted. We will compare it to the definition given by CTTW in terms of the cumulative distribution (or "cumulant" or "radiator") $R\left(\tau_{a}\right)$. We will observe that the two descriptions of $\mathrm{N}^{k} \mathrm{LL}$ are not precisely equivalent. This sets the stage for a more careful prescription for computing to $\mathrm{N}^{k} \mathrm{LL}$ accuracy in the subsequent sections.

In section 3 we consider factorized and resummed event shape distributions predicted in $[24,26]$ using the formalism of SCET and compare to a form based on the predictions of $[12,13]$ derived directly in full QCD. These distributions look similar but not identical at first, but we will put the two into forms that are precisely and transparently equivalent. 
This comparison serves not only a pedagogical purpose, but improves both versions in real ways. The SCET version is put in a form in which more logarithmic terms are explicitly exponentiated and thus sums a larger number of terms to all orders in $\alpha_{s}$ at a given level of logarithmic accuracy. In the QCD version, we generalize the jet and soft scales from the fixed values they usually take to generic, variable forms, whose variation can provide an accurate estimation of theoretical uncertainty at finite $\mathrm{N}^{k} \mathrm{LL}$ accuracy. This restoration of variable scales to the traditional QCD form is a direct byproduct of our proof of its equivalence with the SCET form. This proof in section 3.4 is the technical heart of the paper.

Section 4 contains a detailed pedagogical review of the orders to which one must compute various ingredients of the factorization theorem (hard, jet, soft functions and their anomalous dimensions) to achieve $\mathrm{N}^{k} \mathrm{LL}$ accuracy in event shape cross sections. The counting scheme for the Laplace transform $\widetilde{\sigma}(\nu)$ is standard and straightforward. If we base the definition of $\mathrm{N}^{k} \mathrm{LL}$ accuracy on this object, however, then we argue that in order to evaluate cross section in momentum $\left(\tau_{a}\right)$ space to the equivalent accuracy, some additional care is warranted in evaluating typical formulae for the cumulant $R\left(\tau_{a}\right)$, and even greater care in evaluating the differential distribution $\sigma\left(\tau_{a}\right)$. Naïvely applying the same procedures that one uses to truncate ingredients of the resummed Laplace transform at a given order of accuracy directly to some existing forms for the resummed momentum-space distributions can cause the latter to predict fewer terms at $\mathrm{N}^{k} \mathrm{LL}$ accuracy than one might expect them to. We will give clear prescriptions on how to evaluate the cross sections in momentum space to the equivalent accuracy as the Laplace transforms. Namely, it is preferable to evaluate some quantities in $R\left(\tau_{a}\right)$ to what is known as $\mathrm{N}^{k} \mathrm{LL}^{\prime}$ accuracy (keeping fixed-order jet and soft functions to one higher power in $\alpha_{s}$ than at $\mathrm{N}^{k} \mathrm{LL}$ accuracy) to maintain an equivalent level of accuracy with $\widetilde{\sigma}(\nu)$, and it is essential to evaluate some terms in $\sigma\left(\tau_{a}\right)$ to $\mathrm{N}^{k} \mathrm{LL}^{\prime}$ accuracy, so that an equivalent level of accuracy between $\sigma\left(\tau_{a}\right)$ and the result of taking the derivative of $R\left(\tau_{a}\right)$ is maintained. We will provide a new formula for the resummed differential distribution $\sigma\left(\tau_{a}\right)$ defined in terms of the derivative of the cumulant and to which simple truncation rules can be applied.

Section 5 contains in summary form the results of the study in section 4 , and the reader who wishes to skip to the final formulae and prescriptions for evaluating $\widetilde{\sigma}(\nu), R\left(\tau_{a}\right)$ and $\sigma\left(\tau_{a}\right)$ to $\mathrm{N}^{k} \mathrm{LL}$ or $\mathrm{N}^{k} \mathrm{LL}^{\prime}$ accuracy without working through the details of their development in section 4 should turn directly to section 5 .

In section 6 we will provide some numerical comparisons of formulae in previous literature and in the current paper to illustrate the effect of consistent counting of logarithms and the implementation of the improved procedures we advocate.

Much of the information in this paper is a review of known, existing procedures, and even some of the comparisons we perform have been presented in different contexts in the past (e.g. [29-33], and more recently [34]). For these parts, we hope the reader finds pedagogical value in the unified, coherent explanations we attempt to provide here. However, we believe the new observations we make in comparing SCET and QCD event shape resummation add new practical value as well, increasing the number of resummed terms in one case and scale variability/uncertainty estimation in the other. Moreover, the 
observations we make about how to calculate the momentum-space cumulant to keep it as accurate as the resummed Laplace transform and, more importantly, how to calculate the differential distribution to an accuracy equivalent to that of the cumulant, have not to our knowledge been made clearly and explicitly before. It is our hope that future studies in this direction may avoid the counting complications we identify, so that with knowledge of certain ingredients to a given order in $\alpha_{s}$, they may achieve the greatest accuracy and most realistic uncertainty estimates that are possible. We also hope that the issues resolved here will help ensure that the most precise QCD predictions will be available at the LHC and future facilities.

\section{Orders of resummed perturbative accuracy}

\subsection{Definition of $N^{k} L L$ accuracy: counting in the Laplace exponent}

In this paper we will review and compare methods to resum large logarithms in event shape distributions, expressed in three ways: as a differential cross section, as its Laplace transform, and as a cumulant cross section (also called the radiator, e.g. in [13]):

$$
\begin{aligned}
\sigma(\tau) & \equiv \frac{1}{\sigma_{0}} \frac{d \sigma}{d \tau} \\
\widetilde{\sigma}(\nu) & \equiv \int_{0}^{\infty} d \tau e^{-\nu \tau} \sigma(\tau) \\
R(\tau) & \equiv \int_{0}^{\tau} d \tau^{\prime} \sigma\left(\tau^{\prime}\right),
\end{aligned}
$$

where $\sigma_{0}$ is the Born cross section. Each of the three ways of writing the cross section in eqs. (2.1), (2.2), and (2.3) exhibits logarithms that become large in the two-jet endpoint region $\tau \ll 1$. The differential distribution can be computed as a perturbative expansion in the strong coupling $\alpha_{s}$, for sufficiently large $\tau$. For very small $\tau \lesssim \Lambda_{\mathrm{QCD}} / Q$, the distribution must be convolved with a nonperturbative shape function (e.g. [13, 26, 35-37]) whose effects dominate in this region, but which we do not consider in this paper. The distribution predicted by perturbation theory takes the form,

$$
\begin{aligned}
\sigma(\tau)= & \delta(\tau)+\frac{\alpha_{s}}{4 \pi}\left[a_{12} \mathcal{L}_{1}+a_{11} \mathcal{L}_{0}+a_{10} \delta(\tau)\right] \\
& +\left(\frac{\alpha_{s}}{4 \pi}\right)^{2}\left[a_{24} \mathcal{L}_{3}+a_{23} \mathcal{L}_{2}+a_{22} \mathcal{L}_{1}+a_{21} \mathcal{L}_{0}+a_{20} \delta(\tau)\right] \\
& +\left(\frac{\alpha_{s}}{4 \pi}\right)^{3}\left[a_{36} \mathcal{L}_{5}+a_{35} \mathcal{L}_{4}+a_{34} \mathcal{L}_{3}+a_{33} \mathcal{L}_{2}+a_{32} \mathcal{L}_{1}+a_{31} \mathcal{L}_{0}+a_{30} \delta(\tau)\right] \\
& +\cdots+d\left[\alpha_{s}\right](\tau)
\end{aligned}
$$

where $\mathcal{L}_{n} \equiv \mathcal{L}_{n}(\tau)=\left[\theta(\tau) \ln ^{n} \tau / \tau\right]_{+}$is a plus distribution defined in appendix A, and where $d$ is an integrable function of $\tau$, no more singular than $\ln (1 / \tau)$. Meanwhile the Laplace 


\begin{tabular}{|c|c|c|}
\hline accuracy & $c_{n k}$ & $C_{n}$ \\
\hline $\mathrm{LL}$ & $k=n+1$ & $n=0$ \\
\hline $\mathrm{NLL}$ & $k \geq n$ & $n=0$ \\
\hline $\mathrm{NNLL}$ & $k \geq n-1$ & $n \leq 1$ \\
\hline $\mathrm{N}^{3} \mathrm{LL}$ & $k \geq n-2$ & $n \leq 2$ \\
\hline
\end{tabular}

\begin{tabular}{|c|c|c|}
\hline accuracy & $c_{n k}$ & $C_{n}$ \\
\hline $\mathrm{LL}$ & $k=n+1$ & $n=0$ \\
\hline $\mathrm{NLL}^{\prime}$ & $k \geq n$ & $n=1$ \\
\hline $\mathrm{NNLL}^{\prime}$ & $k \geq n-1$ & $n \leq 2$ \\
\hline $\mathrm{N}^{3} \mathrm{LL}^{\prime}$ & $k \geq n-2$ & $n \leq 3$ \\
\hline
\end{tabular}

Table 1. Conventions for order counting in $\widetilde{\sigma}(\nu)$. At $\mathrm{N}^{k} \mathrm{LL}$ accuracy, all $c_{n k}$ for all $n$ and $m \geq$ $n+k-1$ are included, as are all $C_{n}$ for $n \leq k-1$. In the primed $\mathrm{N}^{k} \mathrm{LL}^{\prime}$ counting, the fixed-order coefficients $C_{n}$ are computed to one higher order in $\alpha_{s}$.

transform and the cumulant both take the form

$$
\begin{aligned}
\{\tilde{\sigma}(\nu), R(\tau)\}= & +\frac{\alpha_{s}}{4 \pi}\left[b_{12} L^{2}+b_{11} L+b_{10}\right] \\
& +\left(\frac{\alpha_{s}}{4 \pi}\right)^{2}\left[b_{24} L^{4}+b_{23} L^{3}+b_{22} L^{2}+b_{21} L+b_{20}\right] \\
& +\left(\frac{\alpha_{s}}{4 \pi}\right)^{3}\left[b_{36} L^{6}+b_{35} L^{5}+b_{34} L^{4}+b_{33} L^{3}+b_{32} L^{2}+b_{31} L+b_{30}\right] \\
& +\cdots+D\left(\alpha_{s}\right),
\end{aligned}
$$

where for the Laplace transform $L=\ln \nu$ while for the cumulant $L=\ln (1 / \tau)$. The remainder function $D$ is either the Laplace transform or the integral of the integrable function $d(\tau)$, with the property $D \rightarrow 0$ as $\tau \rightarrow 0$ or $\nu \rightarrow \infty$. Of course, the coefficients $b_{i j}$ differ between the Laplace transform and the cumulant.

Fixed-order perturbation theory calculates the cross sections in eqs. (2.4) and (2.5) row-by-row, order-by-order in $\alpha_{s}$, which in eqs. (2.4) and (2.5) means $\alpha_{s}(Q)$, where $Q$ is the $\mathrm{CM} e^{+} e^{-}$collision energy. At this high scale, $\alpha_{s} \ll 1$. However when the logs $\mathcal{L}_{n}$ or $L^{n}$ become large, this expansion breaks down. Instead, the expansions should be reorganized so that sets of large logarithms to all orders in $\alpha_{s}$ are summed one set at a time. One might be tempted to sum eqs. (2.4) and (2.5) column-by-column, capturing the largest logs first, then the next-to-largest, etc. However, it turns out that this is not the most systematic way to reorganize the summation. Instead, systematic methods of resummation prefer to sum the logarithm of $\widetilde{\sigma}(\nu)$, which exponentiates in a simple fashion:

$$
\begin{gathered}
\tilde{\sigma}(\nu)=C\left(\alpha_{s}\right) \exp \left\{\frac{\alpha_{s}}{4 \pi}\left[c_{12} L^{2}+c_{11} L\right]\right. \\
+\left(\frac{\alpha_{s}}{4 \pi}\right)^{2}\left[c_{23} L^{3}+c_{22} L^{2}+c_{21} L\right] \\
\left.+\left(\frac{\alpha_{s}}{4 \pi}\right)^{3}\left[c_{34} L^{4}+c_{33} L^{3}+c_{32} L^{2}+c_{31} L\right]+\cdots\right\}+D\left(\alpha_{s}\right), \\
\mathrm{LL}+\mathrm{NLL}+\mathrm{NNLL}+\mathrm{N}^{3} \mathrm{LL}+\cdots
\end{gathered}
$$


where $C$ has an expansion in $\alpha_{s}$ independent of the logarithms,

$$
C\left(\alpha_{s}\right)=1+\frac{\alpha_{s}}{4 \pi} C_{1}+\left(\frac{\alpha_{s}}{4 \pi}\right)^{2} C_{2}+\cdots .
$$

In the expansion in eq. (2.6), the highest power of logs in the exponent at each order $\alpha_{s}^{n}$ is $L^{n+1}$. It turns out that $\widetilde{\sigma}(\nu)$ exponentiates more simply than $\sigma(\tau)$ or $R(\tau)$, and so we use $\widetilde{\sigma}(\nu)$ written as in eq. (2.6) as the benchmark for defining orders of logarithmic accuracy. Systematic methods of resummation give a well-defined, simple definition of this exponent in terms of a limited number of functions in QCD/SCET:

$$
\widetilde{\sigma}(\nu)=C\left(\alpha_{s}\right) e^{\bar{E}(\ln \nu)}+\widetilde{D}\left(\alpha_{s}, \nu\right),
$$

where $\widetilde{D}$ is the non-singular part of $\widetilde{\sigma}$ in Laplace space. We will give a precise definition of $\bar{E}$ in terms of anomalous dimensions and other functions in section 3. We use eq. (2.6) to define the order of accuracy to which we resum logarithms in the cross section: the first column contains all the terms at leading-log (LL) accuracy, the second column nextto-leading $\log$ (NLL), the third next-to-next-to-leading log (NNLL), etc. This counting is natural in a regime where the logs are large enough so that $L \sim 1 / \alpha_{s}$. Then LL sums all terms of order $\alpha_{s}^{-1}$, NLL all terms of order 1, NNLL all terms of order $\alpha_{s}$, etc. In this counting, the fixed-order terms in $C$ are counted as $C_{1}$ being NNLL (i.e. it is explicitly order $\left.\alpha_{s}\right), C_{2}$ being $\mathrm{N}^{3} \mathrm{LL}$, etc. Since the terms in the prefactor $C$ in eq. (2.8) do organize themselves differently than the exponent $\bar{E}$, though, one can adopt an alternative convention for them. Often, the fixed-order coefficients are included to one higher order in $\alpha_{s}$, yielding the so-called "primed" counting [38], summarized in table 1 , so $C_{1}$ would be NLL', $C_{2} \mathrm{NNLL}^{\prime}$, etc. This counting is more useful when one wishes to compute the transition region between small and large $\tau$ to $\mathrm{N}^{k} \mathrm{LO}$ accuracy where the fixed-order terms in the non-singular $D$ are the same size as the logs. In addition, we will find below that in a number of ways the primed counting is a more consistent scheme for counting the accuracy of the resummed logs themselves, at least when computing $R(\tau)$ or $\sigma(\tau)$ in momentum space. When the non-singular terms $D$ are calculated in an ordinary fixed-order expansion to $\mathrm{N}^{n} \mathrm{LO}$ accuracy, etc. we speak of the cross section resummed to $\mathrm{N}^{k} \mathrm{LL}\left({ }^{\prime}\right)$ accuracy and matched onto fixed-order at $\mathrm{N}^{n} \mathrm{LO}$, or, for short, $\mathrm{N}^{k} \mathrm{LL}\left({ }^{\prime}\right)+\mathrm{N}^{n} \mathrm{LO}$.

We will define $\mathrm{N}^{k} \mathrm{LL}$ accuracy for the cumulant $R(\tau)$ or the distribution $\sigma(\tau)$ similarly to $\widetilde{\sigma}(\nu)$ in eq. (2.6). However, because they do not exponentiate as simply as eq. (2.8), the prescriptions for calculating $R(\tau)$ or $\sigma(\tau)$ to $\mathrm{N}^{k} \mathrm{LL}$ (or $\mathrm{N}^{k} \mathrm{LL}^{\prime}$ ) accuracy are a bit more involved. For example, the most compact form for systematic resummation of $R(\tau)$ that we will find below is:

$$
R(\tau)=C\left(\alpha_{s}\right) \exp \left[\bar{E}(\ln 1 / \tau)+\sum_{n=2}^{\infty} \frac{1}{n !} \bar{E}^{(n)} \partial_{\bar{E}^{\prime}}^{n}\right] \frac{1}{\Gamma\left(1-\bar{E}^{\prime}\right)}+D\left(\alpha_{s}, \tau\right),
$$

where $D$ is the non-singular part of $R$ in $\tau$-space. The exponents $\bar{E}$ in eqs. (2.8) and (2.9) take the same form, while the terms generated by the gamma function and its derivatives with respect to $\bar{E}^{\prime}$ [which itself is the derivative $d \bar{E} / d(\ln 1 / \tau)$ ] compensate for the additional terms generated due to the Laplace transform of $\ln ^{n}(1 / \tau)$ being not exactly equal to 
$\ln ^{n}\left(\nu e^{\gamma_{E}}\right)$ (lower order logs are also generated in the transform, as reviewed in appendix B). Thus, to make eq. (2.9) reproduce the entire $\mathrm{N}^{k} \mathrm{LL}$ accurate $\widetilde{\sigma}(\nu)$ in eq. (2.8) exactly requires evaluating the differential operators in eq. (2.9) acting on the gamma function to infinitely high order, for which a closed-form algebraic expression cannot be obtained. However, we will find that keeping the differential operators to sufficiently high finite order, all the terms in $\widetilde{\sigma}(\nu)$ that should be correct at $\mathrm{N}^{k} \mathrm{LL}$ accuracy are indeed reproduced by Laplace transforming $R(\tau)$ in eq. (2.9). We will describe the appropriate procedure and counting in section 4.2.2. Additional similar considerations apply to $\sigma(\tau)$, which we will explain in section 4.3.1.

\subsection{Original CTTW convention: counting in the cumulant}

Resummation of large logarithms in event shape distributions was described by CTTW [15] in terms of the cumulant cross section (also called the "radiator") $R(\tau)$, which can be organized in the form

$$
R(\tau)=C\left(\alpha_{s}\right) \Sigma\left(\tau, \alpha_{s}\right)+D\left(\tau, \alpha_{s}\right)
$$

where

$$
\begin{aligned}
C\left(\alpha_{s}\right)= & +\sum_{n=1}^{\infty}\left(\frac{\alpha_{s}}{2 \pi}\right)^{n} C_{n} \\
\ln \Sigma\left(\tau, \alpha_{s}\right)= & \sum_{n=1}^{\infty} \sum_{m=1}^{n+1}\left(\frac{\alpha_{s}}{2 \pi}\right)^{n} G_{n m} \ln ^{m} \frac{1}{\tau} \\
= & \left(\frac{\alpha_{s}}{2 \pi}\right)\left(G_{12} \ln ^{2} \frac{1}{\tau}+G_{11} \ln \frac{1}{\tau}\right) \\
& +\left(\frac{\alpha_{s}}{2 \pi}\right)^{2}\left(G_{23} \ln ^{3} \frac{1}{\tau}+G_{22} \ln ^{2} \frac{1}{\tau}+G_{21} \ln \frac{1}{\tau}\right) \\
& +\left(\frac{\alpha_{s}}{2 \pi}\right)^{3}\left(G_{34} \ln ^{4} \frac{1}{\tau}+G_{33} \ln ^{3} \frac{1}{\tau}+G_{32} \ln ^{2} \frac{1}{\tau}+G_{31} \ln \frac{1}{\tau}\right)+\cdots,
\end{aligned}
$$

and $D\left(\tau, \alpha_{s}\right)$ is a remainder function that vanishes as $\tau \rightarrow 0$. In eqs. (2.10) and (2.11), $\alpha_{s}$ is again evaluated at the scale $Q$. Expanding $R(\tau)$ in powers of $\alpha_{s}$ explicitly,

$$
R(\tau)=\sum_{n=0}^{\infty} \sum_{m=0}^{2 n} R_{n m}\left(\frac{\alpha_{s}}{2 \pi}\right)^{n} \ln ^{m} \frac{1}{\tau}+D\left(\tau, \alpha_{s}\right) .
$$

In table 2 we give the coefficients $R_{n m}$ in terms of the coefficients $G_{n m}$ in the exponent of $\Sigma$ and coefficients $C_{n}$ in the multiplicative prefactor up to $\mathcal{O}\left(\alpha_{s}^{3}\right)$.

Comparing eqs. (2.10) and (2.11) to eq. (2.9), we note that the CTTW exponent $\ln \Sigma$ is not simply equal to $\bar{E}$, but also contains terms generated by the gamma function $\Gamma\left(1-\bar{E}^{\prime}\right)$ and the derivatives in eq. (2.9) acting on it. Although eq. (2.9) is thus in a slightly more involved mathematical form, in practice it is actually simpler in the sense that eq. (2.9) can be computed from the single object $\bar{E}$ and its derivatives and shows how the CTTW coefficients $G_{n m}$ would be computed systematically to arbitrarily high accuracy. Because eq. (2.9) is not simply an exponential of $\bar{E}$ as in eq. (2.8), we must take care in defining 


\begin{tabular}{|c|c|c|c|}
\hline$R_{n m}$ & $n=1$ & $n=2$ & $n=3$ \\
\hline$m=2 n$ & $R_{12}=G_{12}$ & $R_{24}=\frac{1}{2} G_{12}^{2}$ & $R_{36}=\frac{1}{6} G_{12}^{3}$ \\
\hline$m=2 n-1$ & $R_{11}=G_{11}$ & $R_{23}=G_{23}+G_{12} G_{11}$ & $R_{35}=G_{23} G_{12}+\frac{1}{2} G_{12}^{2} G_{11}$ \\
\hline$m=2 n-2$ & $R_{10}=C_{1}$ & $R_{22}=G_{22}+\frac{1}{2} G_{11}^{2}+C_{1} G_{12}$ & $R_{34}=G_{34}+G_{23} G_{11}+G_{22} G_{12}+\frac{1}{2} G_{12} G_{11}^{2}+\frac{1}{2} C_{1} G_{12}^{2}$ \\
\hline$m=2 n-3$ & - & $R_{21}=G_{21}+C_{1} G_{11}$ & $R_{33}=G_{33}+G_{22} G_{11}+G_{21} G_{12}+\frac{1}{6} G_{11}^{3}+C_{1}\left(G_{23}+G_{12} G_{11}\right)$ \\
\hline$m=2 n-4$ & - & $R_{20}=C_{2}$ & $R_{32}=G_{32}+G_{21} G_{11}+C_{2} G_{12}+C_{1}\left(G_{22}+\frac{1}{2} G_{11}^{2}\right)$ \\
\hline$m=2 n-5$ & - & - & $R_{31}=G_{31}+C_{2} G_{11}+C_{1} G_{21}$ \\
\hline$m=2 n-6$ & - & - & $R_{30}=C_{3}$ \\
\hline
\end{tabular}

Table 2. Coefficients in the fixed-order expansion of radiator $R(\tau) . R_{n m}$ is the coefficient of $\left(\alpha_{s} / 2 \pi\right)^{n} \ln ^{m}(1 / \tau)$ in the expansion of $R(\tau)$, given here up to $\mathcal{O}\left(\alpha_{s}^{3}\right)$ in terms of the coefficients $C_{n}$ and $G_{n m}$ in the exponentiated form of the radiator eq. (2.10).

what $\mathrm{N}^{k} \mathrm{LL}$ accuracy means in evaluating each part of eq. (2.9) in a manner consistent with the prescription for eq. (2.8). This will be the subject of section 4.2.2.

In the presentation of CTTW, $\mathrm{N}^{k} \mathrm{LL}$ accuracy describes the number of terms in the exponent $\ln \Sigma$ of $R$ in eq. (2.11) that are known. We have instead defined $\mathrm{N}^{k} \mathrm{LL}$ accuracy by the number of terms known in the exponent of the Laplace transform $\widetilde{\sigma}(\nu)$ in eq. (2.6). In principle, knowing one set of terms in either of eqs. (2.6) and (2.11b) to $\mathrm{N}^{k} \mathrm{LL}$ accuracy would allow obtaining the other one to the same accuracy by (inverse) Laplace transformation. However, unlike for $R(\tau)$, it is possible to give a closed algebraic form eq. (2.8) for $\widetilde{\sigma}(\nu)$ that predicts all the $\mathrm{N}^{k} \mathrm{LL}$ terms to arbitrarily high order in $\alpha_{s}$ in eq. (2.6). In contrast, eq. (2.9) for $R(\tau)$ does so in principle, but not in a closed algebraic form the exponential of derivatives $\partial_{\bar{E}^{\prime}}^{n}$ cannot actually be evaluated to infinitely high order in practice. So, one has to decide at what finite order in $\alpha_{s}$ to truncate the exponential differential operator to finite order at $\mathrm{N}^{k} \mathrm{LL}$ accuracy. Because of this additional ambiguity or complexity in defining $\mathrm{N}^{k} \mathrm{LL}$ accuracy in terms of $R(\tau)$ (similarly $\sigma(\tau)$ ) in momentum space, we define $\mathrm{N}^{k} \mathrm{LL}$ accuracy instead more simply by the number of terms accurately predicted in the exponent of $\widetilde{\sigma}(\nu)$. We will explain in section 4.2.2 below how to truncate eq. (2.9) for $R(\tau)$ at $\mathrm{N}^{k} \mathrm{LL}$ accuracy properly so that it does in fact match the accuracy of $\tilde{\sigma}(\nu)$.

For the distribution $\sigma(\tau), \mathrm{N}^{k} \mathrm{LL}$ accuracy will be similarly defined, namely, the Laplace transform of the $\mathrm{N}^{k} \mathrm{LL} \sigma(\tau)$ must match the accuracy of the $\mathrm{N}^{k} \mathrm{LL} \tilde{\sigma}(\nu)$. This can be accomplished by simply differentiating $R(\tau)$ computed properly to $\mathrm{N}^{k} \mathrm{LL}$ accuracy or by appropriate truncation of a formula derived for $\sigma(\tau)$ directly. In section 4.3, we will show that care must be exercised in making such truncations of some standard formulae for the resummed distribution. Naïve truncation according to standard rules for $\mathrm{N}^{k} \mathrm{LL}$ accuracy can make those formulae for $\sigma(\tau)$ yield less accurate results than directly differentiating the $\mathrm{N}^{k} \mathrm{LL}$ cumulant. This problem does not exist at $\mathrm{N}^{k} L L^{\prime}$ accuracy.

To close this section, let us comment on counting logs in the fixed-order expansion eq. (2.12) of the cumulant $R(\tau)$. Note that knowing all the coefficients $G_{n k}$ and $C_{n}$ at a given order in $\mathrm{N}^{k} \mathrm{LL}$ accuracy also captures all the terms in the $(k+1)$-th row of the fixedorder expansion of $R(\tau)$ shown in table 2. In the past, this is what was sometimes meant 
by $\mathrm{N}^{k} \mathrm{LL}$ when applied directly to the expansion of $R(\tau)$ in powers of $\alpha_{s}$ (this is what is called " $\mathrm{N}^{k} \mathrm{LL}_{\mathrm{F}}$ " accuracy in [39]). That is, $\mathrm{LL}_{F}$ accuracy means the first row of table 2, $\mathrm{NLL}_{F}$ accuracy means the second row, etc. However, $\mathrm{N}^{k} \mathrm{LL}$ accuracy in the exponent of $R(\tau)$ resums many more terms than this. The number of rows in table 2 do not stand in one-to-one correspondence with the orders of accuracy $\mathrm{N}^{k} \mathrm{LL}$ in table 1 [39]. For resummed calculations, nowadays $\mathrm{N}^{k} \mathrm{LL}$ accuracy invariably refers to the number of terms resummed in the exponent $\ln \Sigma$ (and prefactor $C$ ) of the radiator $R$ (or, rather, in the exponent $\bar{E}$ of the Laplace transform $\widetilde{\sigma}(\nu)$ ). This counting is also much more natural and consistent with systematic calculation using the renormalization group methods that are reviewed in section 3 .

\section{Comparison of direct QCD and SCET resummation}

In this section we review techniques for achieving the resummation of logarithms in eq. (2.6) to all orders in $\alpha_{s}$. On the one hand, none of the techniques reviewed here are new. On the other hand, by gathering them in one place, we will notice some new connections that help generalize or simplify existing results into more illuminating, useful forms, and even improve the accuracy of some resummation formulae. We hope the reader finds new pedagogical and practical value in the combined results we collect and review here.

A variety of techniques have been employed to perform resummation for processes with large logarithms, both within the context of full QCD and also using the tools of effective field theory. For jets, the EFT is SCET. For threshold resummation, these methods have been compared in detail from the perspective of both fields [29, 30, 32-34]). However, for event shapes, such a detailed comparison has not been performed. These techniques are applicable to more complex jet observables [40-44], and will find use in studies of jet substructure [45-47].

In the title of this section we refer to "direct QCD" (dQCD) by which we mean the method of resummation derived directly from the properties of scattering amplitudes in perturbative QCD. This is in contrast to SCET methods derived using the techniques of effective field theory. The two methods differ in details but both describe in a controlled series of approximations the same full theory of QCD. The EFT can be viewed as a systematic method to organize the approximations made in order to factorize cross sections in full QCD rather than being a different theory. The tools of EFT then make systematic the methods of resumming logs via RG evolution. Whether working directly in the full theory or going through the machinery of EFT, the two methods lead to the same resummation results because both arrive at similar factorization of hard, collinear and soft scales for the cross section. In SCET the collinear (jet) and soft functions in the factorization theorem are matrix elements of operators built out of effective theory fields. In direct QCD they rely on perturbative techniques to separate out the subleading contributions that violate the factorization, but can also be expressed as matrix elements of operators similar to those in SCET (see, e.g., $[12,48]$ ). We use the adjective "direct" simply to distinguish whether one uses explicitly the construct of an EFT or not to resum logs. We will, however, often drop the adjective and simply refer to the direct method as "QCD" below. 
We begin this section with a brief overview of factorization in both QCD and SCET, and provide separate short reviews of common resummation techniques in the two formalisms. We then show some rather compact and remarkable ways to rewrite the all-orders resummation formalism that underscore the connection between QCD and SCET. We show that the two methods are equivalent at all orders, and furthermore that, if the resummation is truncated at a given order, the two methods still give the same result. Additionally, we use the connection between the two resummation formalisms to show how non-canonical scale choices can be made in the QCD resummation. This connection also leads us to a form for event shape distributions resummed in SCET that resums more terms than most standard formulae used in the literature to date.

While there is a wide variety of event shapes one can consider (e.g. thrust [7], jet mass [49-51], broadening [52], $C$-parameter [53], $N$-jettiness [42], etc.), a much larger class of event shapes that also encompasses some of these are the angularities [12-14], defined for events in $e^{+} e^{-} \rightarrow$ hadrons as

$$
\tau_{a}=\frac{1}{Q} \sum_{i \in X} E_{i} \sin ^{a} \theta_{i}\left(1-\cos \theta_{i}\right)^{1-a} \stackrel{m_{i}=0}{\longrightarrow} \frac{1}{Q} \sum_{i \in X}\left|\mathbf{p}_{i}^{\perp}\right| e^{-\left|\eta_{i}\right|(1-a)},
$$

where $Q$ is the center-of-mass collision energy, $X$ the hadronic final state, $E_{i}$ the energy of particle $i$, and where the angle $\theta_{i}$, transverse momentum $\mathbf{p}_{i}^{\perp}$ and pseudorapidity $\eta_{i}$ are measured with respect to the thrust axis $\hat{\mathbf{t}}_{X}$ of the final state $X$. The second expression for $\tau_{a}$ holds for massless hadrons; for generalization to nonzero mass, see [8]. The angularities interpolate between thrust at $a=0$ and broadening at $a=1$, although $a$ is allowed to vary between $-\infty<a<2$. The factorization theorem and resummation we discuss below hold for $a<1$. For the case $a=1$ see [54-58]. For "recoil-free" versions of angularities and other event shapes with nice properties at $a=1$, see [59]. Thus, framing our discussion in terms of angularities is not a narrow specialization to a particular variable, but rather a way to encompass many of the above-mentioned event shapes at once in a generic way, and also illustrates how to modify the resummation formulae for observables of different mass dimensions. The discussion below for angularities should be read in this generic light.

\subsection{Factorization and resummation of event shape distributions}

\subsubsection{The factorization theorem}

The cross section for $e^{+} e^{-} \rightarrow$ hadrons differential in the angularity $\tau_{a}$ can be shown to factorize in the two-jet region $\tau_{a} \ll 1$. In this regime, soft and collinear degrees of freedom dominate the final state, and the cross section factorizes into hard, jet, and soft functions $[12,24,26,48]$ :

$$
\frac{d \sigma}{d \tau_{a}}=\sigma_{0} H_{2}\left(Q^{2}, \mu\right) \int d t_{a}^{n} d t_{a}^{\bar{n}} d k_{s} \delta\left(\tau_{a}-\frac{t_{a}^{n}+t_{a}^{\bar{n}}}{Q^{2-a}}-\frac{k_{s}}{Q}\right) J_{n}^{a}\left(t_{a}^{n}, \mu\right) J_{\bar{n}}^{a}\left(t_{a}^{\bar{n}}, \mu\right) S_{2}^{a}\left(k_{s}, \mu\right)
$$

For larger $\tau_{a} \sim 1$, non-singular terms which are power-suppressed in the two-jet region become leading order and must be added to this expression. The factorization theorem eq. (3.2) has been derived both using the methods of direct QCD [12, 48] and SCET [24, $26,48]$. We have changed the notation from that used in $[12,24,26,48]$ so the arguments 
of the jet and soft functions are dimensionful, reflecting the natural quantities on which they depend. As alluded to in section 2, often it is advantageous to study the Laplace transform of eq. (3.2), for which the factorization theorem is

$$
\widetilde{\sigma}\left(\nu_{a}\right)=H_{2}\left(Q^{2}, \mu\right) \widetilde{J}_{n}^{a}\left(\frac{\nu_{a}}{Q^{2-a}}, \mu\right) \widetilde{J}_{\bar{n}}^{a}\left(\frac{\nu_{a}}{Q^{2-a}}, \mu\right) \widetilde{S}_{2}^{a}\left(\frac{\nu_{a}}{Q}, \mu\right),
$$

where

$$
\begin{aligned}
\widetilde{\sigma}\left(\nu_{a}\right) & =\frac{1}{\sigma_{0}} \int_{0}^{\infty} d \tau_{a} e^{-\nu_{a} \tau_{a}} \frac{d \sigma}{d \tau_{a}}, & & \\
\widetilde{J}_{n}^{a}\left(x_{a}, \mu\right) & =\int_{0}^{\infty} d t_{a} e^{-x_{a} t_{a}} J_{n}^{a}\left(t_{a}, \mu\right), & \widetilde{S}_{2}^{a}\left(x_{a}, \mu\right) & =\int_{0}^{\infty} d k_{s} e^{-x_{a} k_{s}} S_{2}^{a}\left(k_{s}, \mu\right), \\
x_{a} & =\frac{\nu_{a}}{Q^{2-a}} \text { for } J, & x_{a} & =\frac{\nu_{a}}{Q} \text { for } S,
\end{aligned}
$$

where for simplicity of notation we let $x_{a}$ take the form appropriate to the function in which it appears. Below, we will generally use $\nu_{a}$ for dimensionless transformed variables and $x_{a}$ for dimensionful ones (with the dimension depending on whether it appears in $J$ or $S$ ). Alternatively one could use the Fourier transforms, for which eq. (3.3) would look similar. We will find, as in previous literature, that the Laplace (or Fourier) transform offers the most straightforward path to defining orders of resummed logarithmic accuracy.

The jet and soft functions in eqs. (3.2) and (3.3) encode the collinear and soft limits of QCD scattering amplitudes and phase space constraints used in [15] to separate jet and soft contibutions to event shape distributions and achieve resummation of logarithms arising from the collinear and soft divergences of QCD. The factorization approach that starts from eqs. (3.2) and (3.3) provides matrix element definitions of these jet and soft functions and is thus very powerful to organize the computation of higher-order perturbative corrections and subleading power corrections [60], and the derivation of general properties of the cross section, such as universality of the leading nonperturbative corrections [8, 48, 61].

We collect here only the basic definitions of the jet and soft functions in eq. (3.2), leaving discussions of their derivation and calculation to the relevant references. The presentation here, which aims at comparing the two formalisms of direct QCD and SCET factorization of the cross section in eqs. (3.2) and (3.3), is very much parallel to that given in [48].

The hard coefficient $H_{2}\left(Q^{2}, \mu\right)$ is a short-distance or hard function containing the underlying partonic hard-scattering diagrams, prior to the collinear branching and soft radiation encoded in the jet and soft functions. It is computed perturbatively and depends on dynamics only at the large energy scale $Q$.

\subsubsection{Jet and soft functions in direct QCD}

In direct $\mathrm{QCD}$, the quark jet functions in eq. (3.2) are defined in terms of matrix elements $[12,48]$

$$
\begin{array}{r}
J_{c}^{\prime \mu}\left(t_{a}^{n}, a, \mu\right)=\frac{2}{Q^{2}} \frac{(2 \pi)^{6}}{N_{C}} \sum_{N_{J_{c}}} \operatorname{Tr}\left[\gamma^{\mu}\left\langle 0\left|\Phi_{\xi_{n}}^{(q) \dagger}(0) q(0)\right| N_{J_{c}}\right\rangle\left\langle N_{J_{c}}\left|\bar{q}(0) \Phi_{\xi_{c}}^{(q)}(0)\right| 0\right\rangle\right] \\
\times \delta\left(t_{a}^{n}-Q^{2-a} \tau_{a}\left(N_{J_{c}}\right)\right) \delta\left(Q-\omega\left(N_{J_{c}}\right)\right) \delta^{2}\left(\hat{n}_{J_{c}}-\hat{n}\left(N_{J_{c}}\right)\right),
\end{array}
$$


where $c=n, \bar{n}$ labels the direction of the jet, $N_{C}$ is the number of colors, the sum is over a set of collinear states $N_{J_{n}}, \tau_{a}\left(N_{J_{c}}\right)$ is the measured angularity of state $N_{J_{c}}$, and $\omega\left(N_{J_{c}}\right)$ its total energy. These jet functions are, in the notation of [12], also differential in the direction $\hat{n}_{J_{c}}$ of the final state jet. In the inclusive event shape distribution eq. (3.2) we integrate over these directions. The scalar jet functions in eq. (3.2) are the projection of eq. (3.5) along the lightlike direction $\beta_{c}$ of the jet, $J_{n}^{a}\left(t_{a}, \mu\right)=\bar{\beta}_{c} \cdot J_{c}^{\prime}\left(t_{a}^{n}, a, \mu\right)$. The jet operators in eq. (3.5) contain the Wilson lines

$$
\Phi_{\xi_{c}}^{(f)}(z)=P \exp \left[i g \int_{-\infty}^{0} d \lambda \xi_{c} \cdot \mathcal{A}^{(f)}\left(\lambda \xi_{c}+z\right)\right],
$$

which are path-ordered exponentials of gluons in the color representation $(f)$ along a 4vector direction $\xi_{c}$, which in [12] was taken to be off the light-cone, at least to start.

The soft function, meanwhile, is defined in direct QCD by starting with an "eikonal" cross section reflecting the eikonal Feynman rules for emissions of soft gluons from energetic partons,

$\bar{\sigma}^{(\mathrm{eik})}\left(k_{s}, \mu\right)=\frac{1}{N_{C}} \sum_{N_{\text {eik }}}\left\langle 0\left|\Phi_{\bar{n}}^{(\bar{q}) \dagger}(0) \Phi_{n}^{(q) \dagger}(0)\right| N_{\text {eik }}\right\rangle\left\langle N_{\text {eik }}\left|\Phi_{n}^{(q)}(0) \Phi_{\bar{n}}^{(\bar{q})}(0)\right| 0\right\rangle \delta\left(k_{s}-Q \tau_{a}\left(N_{\text {eik }}\right)\right)$,

where the final states $N_{\text {eik }}$ are those produced by Wilson lines in the directions $n, \bar{n}$. Again $\tau_{a}\left(N_{\text {eik }}\right)$ is the value of the angularity measured in state $N_{\text {eik }}$. The eikonal cross section provides a good approximation to the soft radiation at large angles from $n, \bar{n}$ but double counts soft radiation along these jet directions that are already in the jet functions eq. (3.5). To avoid this double-counting in the factorization theorem eq. (3.2), one defines a set of eikonal jet functions to be subtracted out of the eikonal cross section:

$$
\begin{aligned}
\bar{J}_{c}^{(\text {eik })}\left(k_{s}, \mu\right)=\frac{1}{N_{C}} \sum_{N_{c}^{\text {eik }}}\left\langle 0\left|\Phi_{\xi_{c}}^{\left(f_{c}\right) \dagger}(0) \Phi_{\beta_{c}}^{\left(f_{c}\right) \dagger}(0)\right| N_{c}^{\text {eik }}\right\rangle\left\langle N_{c}^{\text {eik }}\left|\Phi_{\beta_{c}}^{\left(f_{c}\right)}(0) \Phi_{\xi_{c}}^{\left(f_{c}\right)}(0)\right| 0\right\rangle \\
\times \delta\left(k_{s}-Q \tau_{a}\left(N_{c}^{\text {eik }}\right)\right),
\end{aligned}
$$

where the roles of the quarks in eq. (3.5) are replaced by lightlike Wilson lines. Defining the soft function in Laplace transform space,

$$
\widetilde{S}\left(x_{s}\right)=\frac{\widetilde{\sigma}^{(\text {eik })}\left(x_{s}\right)}{\tilde{J}_{n}^{(\text {eik })}\left(x_{s}\right) \tilde{J}_{\bar{n}}^{\text {eik })}\left(x_{s}\right)},
$$

avoids double counting in the factorized cross section, and leads to the correct factorization theorem eqs. (3.2) and (3.3). In [48] it was argued that subtracting the double-counted contributions out of the jet functions instead leads to definitions of jet and soft functions more parallel to SCET:

$$
\widetilde{\sigma}(\nu)=\sigma_{0}(Q) \widetilde{\mathcal{J}}_{n}\left(x_{a}\right) \widetilde{\mathcal{J}}_{\bar{n}}\left(x_{a}\right) \widetilde{\sigma}^{(\text {eik })}\left(x_{a}\right),
$$

where

$$
\widetilde{\mathcal{J}}_{c}\left(x_{a}\right)=\frac{\tilde{J}_{c}\left(x_{a}\right)}{\tilde{J}_{c}^{(\mathrm{eik})}\left(x_{a}\right)} .
$$

This organization is directly related to the method of "zero-bin subtraction" in SCET [62], a relation which was discussed in some detail in [48, 63, 64] 


\subsubsection{Jet and soft functions in SCET}

The jet and soft functions in SCET have similar definitions as above. Some differences in approach are that SCET begins with a Lagrangian built out of collinear and soft quark and gluon fields, formed after integrating out hard modes at the scale $\mu \sim Q$. This Lagrangian encodes the Feynman rules for evaluating matrix elements of collinear or soft fields. Details of the derivation of the leading-order Lagrangian can be found in [19,20], the decoupling of soft and collinear modes in the theory in [21]. The factorization in eq. (3.2) of event shape distributions in the two-jet region proceeds by matching the quark electroweak current in QCD onto currents of collinear and soft operators in SCET, the details of which can be found to $\mathcal{O}\left(\alpha_{s}\right)$ accuracy in $[22,31]$. Details of the proof of the factorization are found in $[22,24]$.

It is not our intent to review all of these details here, but only to give the definitions of the jet and soft functions in SCET arising from the proof of eq. (3.2) found in the above references. The collinear quark jet functions appearing in eq. (3.2) are defined in SCET in terms of matrix elements of collinear jet operators,

$J_{n}^{a}\left(t_{a}^{n}, \mu\right)=\int \frac{d l^{+}}{2 \pi} \frac{1}{2 N_{C}} \operatorname{Tr} \int d^{4} x e^{i l \cdot x}\left\langle 0\left|\frac{\not h}{2} \chi_{n}(x) \delta\left(t_{a}^{n}-Q^{2-a} \hat{\tau}_{a}^{n}\right) \delta(Q+\bar{n} \cdot \mathcal{P}) \delta^{2}\left(\mathcal{P}_{\perp}\right) \bar{\chi}_{n}(0)\right| 0\right\rangle$,

where the trace is over colors and Dirac indices, $\mathcal{P}^{\mu}$ is a "label" momentum operator picking out the large components of the momentum of the collinear modes $\chi_{n}$ [20], and $\hat{\tau}_{a}^{n}$ is an operator measuring the angularity of final states in the cut diagrams that must be evaluated to compute the matrix element in eq. (3.12) [24]. The collinear jet fields $\chi_{n}$ are themselves built out of collinear quark fields and collinear Wilson lines in SCET:

$$
\chi_{n}(x)=\sum_{\tilde{p}} \chi_{n, \tilde{p}}(x), \quad \chi_{n, \tilde{p}}=\left[\delta(\omega-\bar{n} \cdot \mathcal{P}) \delta^{2}\left(\tilde{p}_{\perp}-\mathcal{P}_{\perp}\right) W_{n}^{\dagger} \xi_{n}\right]
$$

where $\tilde{p}^{\mu}=\omega n^{\mu} / 2+\tilde{p}_{\perp}^{\mu}$ is the large label momentum of the jet field $\chi_{n}$, and $\xi_{n}$ is a collinear quark field, and $W_{n}$ is the Wilson line of collinear gluons,

$$
W_{n}(x)=\sum_{\text {perms }} \exp \left[-\frac{g}{\bar{n} \cdot \mathcal{P}} \bar{n} \cdot A_{n}(x)\right]
$$

where $A_{n}^{\mu}(x)=\sum_{\tilde{p}} A_{n, p}^{\mu}(x)$ is an $n$-collinear gluon field.

Meanwhile the soft function in eq. (3.2) is a matrix element of soft gluon Wilson lines,

$$
S\left(k_{s}, \mu\right)=\frac{1}{N_{C}} \operatorname{Tr}\left\langle 0\left|\bar{Y}_{\bar{n}}^{\dagger}(0) Y_{n}^{\dagger}(0) \delta\left(k_{s}-Q \hat{\tau}_{a}^{s}\right) Y_{n}(0) \bar{Y}_{\bar{n}}(0)\right| 0\right\rangle,
$$

where the trace is over colors, $\hat{\tau}_{a}^{s}$ is an operator [24] measuring the angularity $\tau_{a}$ of soft final states, and the Wilson lines are defined

$$
Y_{n}(x)=P \exp \left[i g \int_{0}^{\infty} d s n \cdot A_{s}(n s+x)\right], \quad \bar{Y}_{\bar{n}}(x)=P \exp \left[i g \int_{0}^{\infty} d s \bar{n} \cdot \bar{A} \bar{s}_{s}(\bar{n} s+x)\right],
$$

where $A_{s}$ and $\bar{A}_{s}$ are soft gluons in the fundamental and anti-fundamental representation, respectively [22]. The Wilson lines $Y_{n, \bar{n}}$ in eq. (3.15) arise by a field redefinition of the 
collinear fields in the SCET Lagrangian that achieves a decoupling of soft and collinear interactions [21].

The jet and soft functions in SCET must also be carefully defined to avoid double counting of soft radiation that happens to go along the collinear directions $n, \bar{n}$. In SCET this is achieved by a "zero-bin subtraction" [62] that accounts for the fact that the sums over collinear label momenta in equations like eq. (3.13) do not include the momentum bin $\tilde{p}=0$. Since this subtraction is performed out of the collinear functions, it is akin to the direct QCD scheme in eqs. (3.10) and (3.11). This equivalence was discussed in [48, 63, 64].

The factorization theorems eqs. (3.2) and (3.3) separate the dependence on the hard, jet, and soft scales whose ratios appear in the arguments of the large logarithms of $\tau_{a}$ in the QCD cross section. In the hard, jet, and soft functions in dimensional regularization, the logs are of ratios of the scale $\mu$ over a single scale, the hard scale $Q$, the jet scale $Q \tau_{a}^{1 /(2-a)}$, or the soft scale $Q \tau_{a}$. The RG evolution of each of these functions with $\mu$ is what allows for systematic resummation of the large logarithms in the cross section. We review below how this is done in both methods, first in SCET and then in direct QCD.

\subsection{Resummed event shape distributions in SCET}

\subsubsection{Perturbative expansions and evolution of hard, jet, and soft functions}

The hard function $H_{2}$ in eq. (3.2) is given by $H_{2}\left(Q^{2}, \mu\right)=\left|C_{2}\left(Q^{2}, \mu\right)\right|^{2}$, where $C_{2}$ is the Wilson coefficient describing the matching from QCD onto the 2-jet operator $\mathrm{O}_{2}$ in SCET, which comes from integrating out the short distance, energetic modes in QCD capable of creating energetic jets, and is observable independent [17]. To $\mathcal{O}\left(\alpha_{s}\right)$, the hard function is given by $[22,31]$

$$
H_{2}\left(Q^{2}, \mu\right)=1+\frac{\alpha_{s}(\mu) C_{F}}{4 \pi}\left(-16+\frac{7 \pi^{2}}{3}-12 \ln \frac{\mu}{Q}-8 \ln ^{2} \frac{\mu}{Q}\right),
$$

and is known to three loops $[29,65,66]$. Each jet function $J_{n}^{a}$ comes from collinear radiation in a jet, and is given to $\mathcal{O}\left(\alpha_{s}\right)$ by [26]:

$$
\begin{aligned}
J_{n, \bar{n}}^{a}\left(t_{a}, \mu\right)= & \delta\left(t_{a}\right)\left[1+\frac{\alpha_{s}(\mu) C_{F}}{4 \pi} f(a)\right] \\
& +\frac{\alpha_{s}(\mu) C_{F}}{4 \pi} \frac{1}{2-a}\left[-\frac{6}{\mu^{2-a}} \mathcal{L}_{0}\left(\frac{t_{a}}{\mu^{2-a}}\right)+\frac{8}{1-a} \frac{1}{\mu^{2-a}} \mathcal{L}_{1}\left(\frac{t_{a}}{\mu^{2-a}}\right)\right],
\end{aligned}
$$

where

$f(a)=\frac{1}{2-a}\left(14-13 a-\frac{\pi^{2}}{6} \frac{12-20 a+9 a^{2}}{1-a}-4 \int_{0}^{1} d x \frac{2-2 x+x^{2}}{x} \ln \left[(1-x)^{1-a}+x^{1-a}\right]\right)$,

which reduces to $f(0)=7-\pi^{2}$ for $a=0$, agreeing with the one-loop result for the standard jet function $J^{0}\left(t_{0}\right)[67,68]$. The jet function depends naturally on a $(2-a)$-dimensional variable $t_{a}$. For $a=0$ the jet function is known to two loops [68] and the anomalous dimension to three loops [29]. Meanwhile, the soft function $S_{2}^{a}$ describes the global soft radiation over the entire event, and is given to $\mathcal{O}\left(\alpha_{s}\right)$ by [26]:

$$
S_{2}^{a}(k, \mu)=\delta(k)\left(1+\frac{\alpha_{s}(\mu) C_{F}}{4 \pi} \frac{1}{1-a} \frac{\pi^{2}}{3}\right)-\frac{\alpha_{s}(\mu) C_{F}}{4 \pi} \frac{16}{1-a} \frac{1}{\mu} \mathcal{L}_{1}\left(\frac{k}{\mu}\right) .
$$




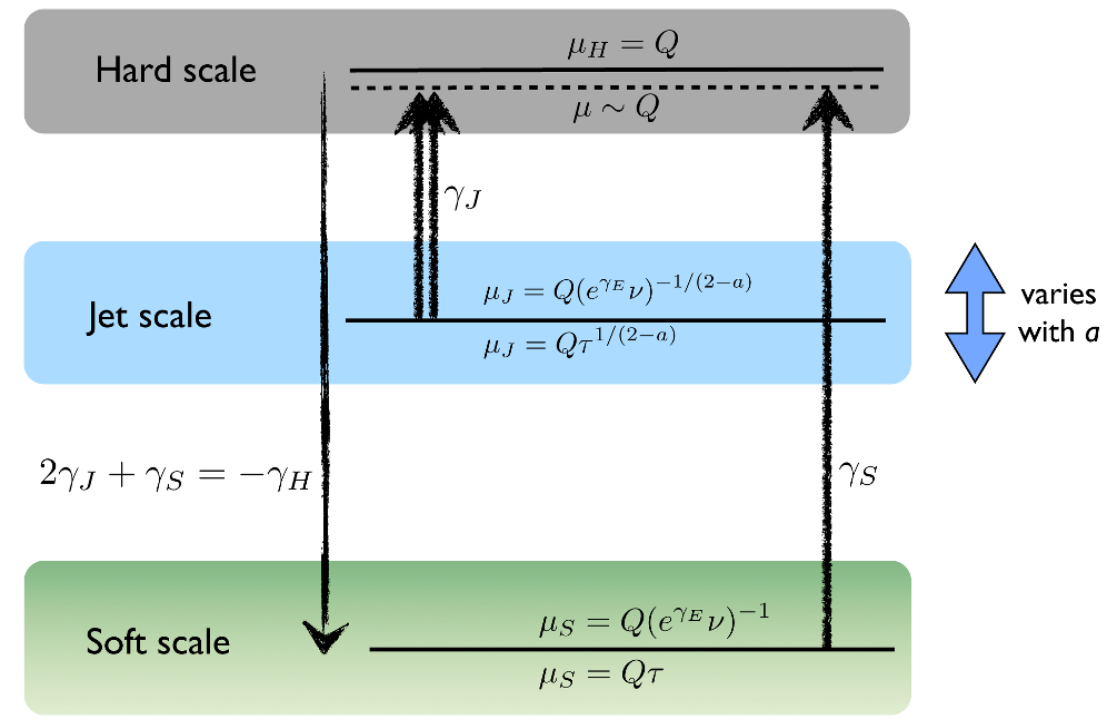

Figure 1. Natural scales for angularity event shapes in the factorization theorem eq. (3.2). The hard, jet, and soft scales in this ladder minimize logs in the hard, jet, and soft functions in the angularity cross sections eq. (3.48) in $\tau_{a}$ space or eq. (4.1) in Laplace space. The common factorization scale $\mu$ in eq. (3.2) can be chosen anywhere, but is commonly chosen near $\mu \sim Q$. The functions are evolved via RGEs from their natural scales to $\mu$. The anomalous dimensions satisfy the consistency condition $\gamma_{H}+2 \gamma_{J}+\gamma_{S}=0$. Note that the jet scale varies with $a$. At $a=1$ it coincides with the soft scale, and a new factorization theorem is required to sum logs in the jet and soft functions, e.g. using $\mathrm{SCET}_{\text {II }}$ [54-57] or using "recoil-free" versions of angularities [59].

$S_{2}^{a}$ is a function of the sum of momenta $k=n \cdot k_{S}^{A}+\bar{n} \cdot k_{S}^{B}$, the sum of soft momenta in the hemispheres $A, B$ projected onto the $n, \bar{n}$ directions that determine the two hemispheres. For $a=0$ the soft function is known to two loops [5, 69, 70], and the three-loop anomalous dimension can be obtained from the three-loop hard and jet anomalous dimensions by the requirement of RG invariance of the cross section eq. (3.2) [71]. The distributions $\mathcal{L}_{n}$ in eqs. (3.18) and (3.20) are "plus distributions":

$$
\mathcal{L}_{n}(x) \equiv\left[\frac{\theta(x) \ln ^{n} x}{x}\right]_{+},
$$

and are defined in appendix A.

The different functions in the factorization theorem each describe physics at different scales, and the natural scales associated with these functions are well separated. Ratios of these scales produce the $\operatorname{logs}$ of $\tau_{a}$ in the QCD cross section. The factorization theorem eq. (3.2) splits up these logs into logs of the factorization scale $\mu$ over only one of these natural scales at a time. The one-loop results in eqs. (3.17), (3.18), and (3.20) for the hard, jet and soft functions display this natural scale dependence illustrated in figure 1. After plugging them into the factorized cross section eq. (3.2), these scales are identified to be:

$$
\mu_{H}^{\mathrm{nat}}=Q, \quad \mu_{J}^{\mathrm{nat}}=Q \tau_{a}^{1 /(2-a)}, \quad \mu_{S}^{\mathrm{nat}}=Q \tau_{a} .
$$


Large logarithms of the ratios of these scales to the factorization scale $\mu$ are produced in the fixed-order expansions of these functions, and these logs must be resummed to obtain accurate predictions in the small $\tau_{a}$ regime. The renormalization group (RG) allows us to independently evolve each function in $\mu$ and sum these logs. Each function has an associated RG equation relating the renormalized function to its anomalous dimension:

$$
\begin{aligned}
\frac{d}{d \ln \mu} H_{2}(\mu) & =\gamma_{H}(\mu) H_{2}(\mu), \\
\frac{d}{d \ln \mu} J_{i}^{a}\left(t_{a}, \mu\right) & =\int d t_{a}^{\prime} \gamma_{J}^{a}\left(t_{a}-t_{a}^{\prime}, \mu\right) J_{i}^{a}\left(t_{a}^{\prime}, \mu\right), \\
\frac{d}{d \ln \mu} S_{2}^{a}(k, \mu) & =\int d k^{\prime} \gamma_{S}^{a}\left(k-k^{\prime}, \mu\right) S_{2}^{a}\left(k^{\prime}, \mu\right) .
\end{aligned}
$$

The hard function anomalous dimension takes the form

$$
\gamma_{H}(\mu)=\kappa_{H} \Gamma_{\text {cusp }}^{q}\left[\alpha_{s}\right] \ln \left(\frac{Q}{\mu}\right)+\gamma_{H}\left[\alpha_{s}\right]
$$

dependent on "cusp" and "non-cusp" anomalous dimensions $\Gamma_{\text {cusp }}^{q}\left[\alpha_{s}\right], \gamma_{H}\left[\alpha_{s}\right]$. The jet and soft functions have a common form for the anomalous dimension:

$$
\gamma_{F}\left(t_{F}, \mu\right)=\kappa_{F} \Gamma_{\text {cusp }}^{q}\left[\alpha_{s}\right] \frac{1}{\mu^{j_{F}}} \mathcal{L}_{0}\left(\frac{t_{F}}{\mu^{j_{F}}}\right)+\gamma_{F}\left[\alpha_{s}\right] \delta\left(t_{F}\right),
$$

where $F=J, S$ for the jet and soft functions, and $t_{F}$ is a variable of mass dimension $j_{F}$, equal to $t_{a}$ for the jet function and $k$ for the soft function.

The cusp anomalous dimension $\Gamma_{\text {cusp }}^{q}\left[\alpha_{s}\right]$ is a universal series in $\alpha_{s}$,

$$
\Gamma_{\text {cusp }}^{q}\left[\alpha_{s}\right]=\sum_{n=0}^{\infty}\left(\frac{\alpha_{s}}{4 \pi}\right)^{n+1} \Gamma_{n}^{q},
$$

where the coefficients are given up to $n=2$, or $\mathcal{O}\left(\alpha_{s}^{3}\right)$, by [72, 73]:

$$
\begin{aligned}
& \Gamma_{0}^{q}=4 C_{F} \\
& \Gamma_{1}^{q}=\Gamma_{0}^{q}\left[\left(\frac{67}{9}-\frac{\pi^{2}}{3}\right) C_{A}-\frac{20}{9} T_{F} n_{f}\right] \\
& \Gamma_{2}^{q}=\Gamma_{0}^{q}\left[\left(\frac{245}{6}-\frac{134 \pi^{2}}{27}+\frac{11 \pi^{4}}{45}+\frac{22 \zeta_{3}}{3}\right) C_{A}^{2}+\left(-\frac{418}{27}+\frac{40 \pi^{2}}{27}-\frac{56 \zeta_{3}}{3}\right) C_{A} T_{F} n_{f}\right. \\
&\left.+\left(-\frac{55}{3}+16 \zeta_{3}\right) C_{F} T_{F} n_{f}-\frac{16}{27} T_{F}^{2} n_{f}^{2}\right]
\end{aligned}
$$

The non-cusp anomalous dimension is similarly defined, with coefficients $\gamma_{n}^{F}$ that are specific to each function. The constants $\kappa_{F}$ give the proportionality of the cusp part of each

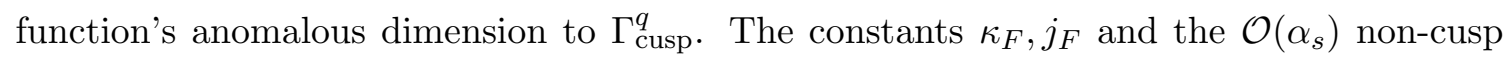
anomalous dimensions are

$$
\begin{array}{lll}
j_{H}=1, & \kappa_{H}=4, & \gamma_{H}^{0}=-12 C_{F}, \\
j_{J}=2-a, & \kappa_{J}=-\frac{2}{1-a}, & \gamma_{J}^{0}=6 C_{F}, \\
j_{S}=1, & \kappa_{S}=\frac{4}{1-a}, & \gamma_{S}^{0}=0 .
\end{array}
$$


For completeness, we include a constant $j_{H}$ for the hard function in this table, which is implicitly the mass dimension of $Q$ in eq. (3.24), and which we henceforth always set equal to 1 . Similarly we will always set $j_{S}=1$ except in equations where we refer to a generic $j_{F}$. We define the "cusp part" of each function's anomalous dimension by

$$
\Gamma_{F} \equiv-\frac{j_{F} \kappa_{F}}{2} \Gamma_{\text {cusp }}^{q},
$$

for notational use below. The non-cusp piece of each function's anomalous dimension is given by an expansion

$$
\gamma_{F}\left[\alpha_{s}\right]=\sum_{n=0}^{\infty}\left(\frac{\alpha_{s}}{4 \pi}\right)^{n+1} \gamma_{F}^{n},
$$

where $F=H, J, S$. These coefficients are given to $\mathcal{O}\left(\alpha_{s}^{3}\right)$ for the hard function and the $a=0$ jet and soft functions in appendix C. For $a \neq 0$ they are so far known only to $\mathcal{O}\left(\alpha_{s}\right)[26]$

The convolutions in the RGEs in eq. (3.23) for the jet and soft functions in momentum space can be removed by Fourier or Laplace transforming the functions, in which case the evolution is multiplicative (as in the hard function). The evolution is performed in the transform space, and the result is transformed back to momentum space (e.g. [74, 75]).

Two formalisms exist in SCET to write the evolution in momentum space [29, 30, 76], each with its own advantages. One method, described in ref. [76], performs the transformation of the jet/soft function solutions in Laplace/position space back to momentum space, and then writes the result for the resummed cross section as a convolution between momentum-space fixed-order jet/soft functions and momentum-space evolution factors. This method requires calculation of convolutions of plus functions with each other, all explicitly computed in [76]. This is particularly useful for the types of explicit calculations in, e.g., [38, 76]. The other method, used in ref. [29, 30], writes the evolution in terms of a derivative operator built out of Laplace-space jet/soft functions acting on a simple $\tau$-dependent function. This formalism turns out to be more transparently relatable to the direct QCD resummation formalism used in, e.g., [12, 13, 77], and is somewhat more compact to write down. For the purposes of our study, we find it more convenient to employ the latter formalism. The two methods, as long as formulae are truncated appropriately, yield numerically equivalent results, as illustrated in, e.g., [78].

\subsubsection{Evolution in SCET}

Taking the Laplace (similarly, Fourier) transform of the jet function using the variable $x_{a}$ conjugate to $t_{a}$ makes the evolution equation multiplicative,

$$
\frac{d}{d \ln \mu} \widetilde{J}\left(x_{a}, \mu\right)=\widetilde{\gamma}_{J}\left(x_{a}, \mu\right) \widetilde{J}\left(x_{a}, \mu\right)
$$

and analogously for the soft function. The Laplace-transformed anomalous dimensions have the form, for $F=J, S$,

$$
\widetilde{\gamma}_{F}\left(x_{a}, \mu\right)=-\kappa_{F} \Gamma_{\text {cusp }}^{q}\left[\alpha_{s}\right] \ln \left(\mu^{j_{F}} x_{a} e^{\gamma_{E}}\right)+\gamma_{F}\left[\alpha_{s}\right],
$$


where $x_{a}$ is dimension $-2+a$ for $F=J$, and dimension -1 for $F=S$. A multiplicative RG equation, as in eq. (3.31), leads to a straightforward evolution equation that allows us to write the function at an arbitrary scale (in terms of its value at another arbitrary scale),

$$
\widetilde{F}\left(x_{a}, \mu\right)=\widetilde{F}\left(x_{a}, \mu_{0}\right) \exp \left(\int_{\mu_{0}}^{\mu} \frac{d \mu^{\prime}}{\mu^{\prime}} \widetilde{\gamma}_{F}\left(x_{a}, \mu^{\prime}\right)\right) .
$$

To perform the evolution we define three functions: ${ }^{1}$

$$
\begin{aligned}
K_{\Gamma}\left(\mu, \mu_{0}\right) & \equiv \int_{\mu_{0}}^{\mu} \frac{d \mu^{\prime}}{\mu^{\prime}} \Gamma_{\text {cusp }}^{q}\left[\alpha_{s}\left(\mu^{\prime}\right)\right] \ln \frac{\mu^{\prime}}{\mu_{0}} \\
\eta_{\Gamma}\left(\mu, \mu_{0}\right) & \equiv \int_{\mu_{0}}^{\mu} \frac{d \mu^{\prime}}{\mu^{\prime}} \Gamma_{\text {cusp }}^{q}\left[\alpha_{s}\left(\mu^{\prime}\right)\right] \\
K_{\gamma_{F}}\left(\mu, \mu_{0}\right) & \equiv \int_{\mu_{0}}^{\mu} \frac{d \mu^{\prime}}{\mu^{\prime}} \gamma_{F}\left[\alpha_{s}\left(\mu^{\prime}\right)\right] .
\end{aligned}
$$

These separate the evolution of the function between two arbitrary scales $\left(\mu_{0}, \mu\right)$ from the dependence on the particular scale $x_{a}^{1 / j_{F}}$ in the anomalous dimension,

$$
\int_{\mu_{0}}^{\mu} \frac{d \mu^{\prime}}{\mu^{\prime}} \widetilde{\gamma}_{F}\left(\mu^{\prime}\right)=-j_{F} \kappa_{F} K_{\Gamma}\left(\mu, \mu_{0}\right)+K_{\gamma_{F}}\left(\mu, \mu_{0}\right)-\kappa_{F} \eta_{\Gamma}\left(\mu, \mu_{0}\right) \ln \left(\mu_{0}^{j_{F}} e^{\gamma_{E}} x_{a}\right) .
$$

We define

$$
\begin{aligned}
K_{F}\left(\mu, \mu_{0}\right) & \equiv-j_{F} \kappa_{F} K_{\Gamma}\left(\mu, \mu_{0}\right)+K_{\gamma_{F}}\left(\mu, \mu_{0}\right), \\
\omega_{F}\left(\mu, \mu_{0}\right) & \equiv-\kappa_{F} \eta_{\Gamma}\left(\mu, \mu_{0}\right),
\end{aligned}
$$

so that

$$
\widetilde{F}\left(x_{a}, \mu\right)=\widetilde{F}\left(x_{a}, \mu_{0}\right) \exp \left(K_{F}\left(\mu, \mu_{0}\right)\right)\left(\mu_{0}^{j_{F}} e^{\gamma_{E}} x_{a}\right)^{\omega_{F}\left(\mu, \mu_{0}\right)} .
$$

For the hard function, the evolution equation reads

$$
H(\mu)=H\left(\mu_{0}\right) \exp \left(K_{H}\left(\mu, \mu_{0}\right)\right)\left(\frac{\mu_{0}}{Q}\right)^{j_{H} \omega_{H}\left(\mu, \mu_{0}\right)} .
$$

Using $K_{F}$ and $\omega_{F}$ has factored out the $x_{a}$ dependence in the evolution factor in eq. (3.37), and we can perform the inverse Laplace transform to obtain the evolution relation in momentum space:

$$
F\left(t_{F}, \mu\right)=\int d t_{F}^{\prime} U_{F}\left(t_{F}-t_{F}^{\prime}, \mu, \mu_{0}\right) F\left(t_{F}^{\prime}, \mu_{0}\right),
$$

where the evolution kernel $U_{F}$ is

$$
U_{F}\left(t_{F}, \mu, \mu_{0}\right)=\frac{\exp \left(K_{F}\left(\mu, \mu_{0}\right)+\gamma_{E} \omega_{F}\left(\mu, \mu_{0}\right)\right)}{\Gamma\left(1-\omega_{F}\left(\mu, \mu_{0}\right)\right)}\left[-\frac{\omega_{F}}{\mu_{0}^{j_{F}}} \mathcal{L}^{-\omega_{F}}\left(\frac{t_{F}}{\mu_{0}^{j_{F}}}\right)+\delta\left(t_{F}\right)\right]
$$

\footnotetext{
${ }^{1}$ Note that these can also be defined as integrals over the running coupling [see eq. (4.18)] by using the defining relation eq. (4.14) of the beta function, $\beta\left[\alpha_{s}(\mu)\right]=\frac{d}{d \ln \mu} \alpha_{s}(\mu)$. In this case the large logarithms are expressed as logarithms of ratios of $\alpha_{s}$ evaluated at parametrically different scales, while in the definitions of eq. (3.34) the logarithms appear explicitly.
} 
and the distribution $\mathcal{L}^{-\omega}$ is given by the plus distribution [76]

$$
\mathcal{L}^{-\omega}(x)=\left[\frac{\theta(x)}{x^{1+\omega}}\right]_{+},
$$

defined in appendix A. The evolution factor is convolved with the fixed-order expansion of $F\left(t_{F}, \mu_{0}\right)$ to produce $F\left(t_{F}, \mu\right)$. These convolutions produce functions of $\omega_{F}$ multiplying distributions $\mathcal{L}^{\omega_{F}}$, and the jet and soft functions in the factorization theorem can then be convolved to produce the total cross section. The necessary convolutions have been worked out in generality in ref. [76], meaning the procedure is formulaic.

An alternative way to write the evolution uses the following approach illustrated in ref. $[29,30]$. This approach turns out to be more transparently relatable to the dQCD literature and is slightly more compact to write down. First one notes that the $x_{a}$-dependence in the evolution factor in eq. (3.37) is contained entirely in $\operatorname{logs} L_{F}\left(\mu_{0}\right)$, where

$$
L_{F}(\mu) \equiv \ln \left(\mu^{j_{F}} e^{\gamma_{E}} x_{a}\right)=\ln \left[\left(\frac{\mu}{Q}\right)^{j_{F}} e^{\gamma_{E}} \nu\right] .
$$

In the last equality we have indicated what the variable $x_{a}$ gets replaced by when these logs appear in the jet or soft functions in eq. (3.3). Therefore, we can rewrite the $x$-dependence in terms of logarithms $L_{F}(\mu)$. Cleverly, this means we can generate the $L_{F}$ dependence through partial derivatives with respect to $\omega_{F}$. Rewriting eq. (3.37) for $\widetilde{F}$ in this way,

$$
\begin{aligned}
\widetilde{F}\left(L_{F}(\mu), \mu\right) & =\widetilde{F}\left(L_{F}\left(\mu_{0}\right), \mu_{0}\right) \exp \left[K_{F}\left(\mu, \mu_{0}\right)\right] \exp \left[L_{F}\left(\mu_{0}\right) \omega_{F}\left(\mu, \mu_{0}\right)\right] \\
& =\widetilde{F}\left(\partial_{\omega_{F}}, \mu_{0}\right) \exp \left[K_{F}\left(\mu, \mu_{0}\right)\right] \exp \left[L_{F}\left(\mu_{0}\right) \omega_{F}\left(\mu, \mu_{0}\right)\right] .
\end{aligned}
$$

This removes the functional $x_{a}$-dependence in $\widetilde{F}$ at $\mu_{0}$, meaning the inverse transform can be performed completely (without the remaining convolution in eq. (3.39)). The result is

$$
F\left(t_{F}, \mu\right)=\widetilde{F}\left(\partial_{\omega_{F}}, \mu_{0}\right) \exp \left[K_{F}\left(\mu, \mu_{0}\right)\right]\left(\frac{\mu_{0}^{j_{F}}}{t_{F}}\right)^{\omega_{F}\left(\mu, \mu_{0}\right)} \frac{1}{t_{F}} \frac{e^{\gamma_{E} \omega_{F}\left(\mu, \mu_{0}\right)}}{\Gamma\left[-\omega_{F}\left(\mu, \mu_{0}\right)\right]} .
$$

This form deserves some remarks. In the position-space function $\widetilde{F}$ at the initial scale $\mu_{0}$, the $\tau$-dependence $\left(t_{F}\right.$ dependence) is generated via derivatives acting on the resummed series $\omega_{F}$ in the evolution factor. Operationally, the derivatives can be thought of as representing a replacement rule, since we know what functions they are acting on, e.g. eq. (4.33) below. We will see a similar method used in the QCD formulation of the cross section in section 3.3. With jet and soft functions written in the form eq. (3.44) convolution over the momentum variables in eq. (3.2) is quite simple to carry out, leading to a compact form for the resummed cross section.

\subsubsection{Resummed cross section in SCET}

In position or Laplace space, the factorized cross section eq. (3.3) using RG evolved hard, jet, and soft functions takes the form

$$
\begin{aligned}
\widetilde{\sigma}\left(\nu_{a}\right)= & H_{2}\left(Q^{2}, \mu_{H}\right) \widetilde{J}\left(L_{J}\left(\mu_{J}\right), \mu_{J}\right)^{2} \widetilde{S}\left(L_{S}\left(\mu_{S}\right), \mu_{S}\right) \\
& \times \exp \left(K_{H}+2 K_{J}+K_{S}\right)\left(\frac{\mu_{H}}{Q}\right)^{\omega_{H}}\left(\frac{\mu_{J}\left(\nu_{a} e^{\gamma_{E}}\right)^{1 / j_{J}}}{Q}\right)^{2 j_{J} \omega_{J}}\left(\frac{\mu_{S} \nu_{a} e^{\gamma_{E}}}{Q}\right)^{\omega_{S}},
\end{aligned}
$$


Taking the inverse Laplace transform of eq. (3.45), or explicitly carrying through the convolutions in eq. (3.2) using the solutions eq. (3.44) for the momentum-space jet and soft functions, the momentum-space cross section is

$$
\begin{aligned}
\sigma\left(\tau_{a}\right)=\exp \left(K_{H}+\right. & \left.2 K_{J}+K_{S}\right)\left(\frac{\mu_{H}}{Q}\right)^{\omega_{H}}\left(\frac{\mu_{J}}{Q \tau_{a}^{1 / j_{J}}}\right)^{2 j_{J} \omega_{J}}\left(\frac{\mu_{S}}{Q \tau_{a}}\right)^{\omega_{S}} H_{2}\left(Q^{2}, \mu_{H}\right) \\
& \times \widetilde{J}\left(\partial_{\Omega}+\ln \frac{\mu_{J}^{j_{J}}}{Q^{j_{J}} \tau_{a}}, \mu_{J}\right)^{2} \widetilde{S}\left(\partial_{\Omega}+\ln \frac{\mu_{S}}{Q \tau_{a}}, \mu_{S}\right) \frac{1}{\tau_{a}} \frac{\exp \left(\gamma_{E} \Omega\right)}{\Gamma(-\Omega)},
\end{aligned}
$$

where

$$
\Omega \equiv 2 \omega_{J}+\omega_{S}
$$

The result eq. (3.46) for the thrust $(a=0)$ appeared in a very similar form in [71] and for arbitrary $a$ in [26], following the methods in [29, 30]. In eq. (3.46), we have pulled through each ratio of factorization scales $\mu_{H}, \mu_{J}$, and $\mu_{S}$ to the canonical scales in eq. (3.22) past the fixed-order functions $\widetilde{J}$ and $\widetilde{S}$. This shifts the derivative in each function by a logarithm of the relevant scale ratio. Integrating eq. (3.46) to yield the cumulant $R\left(\tau_{a}\right)$ defined in eq. (2.3) is simple,

$$
\begin{aligned}
R\left(\tau_{a}\right)=\exp & \left(K_{H}+2 K_{J}+K_{S}\right)\left(\frac{\mu_{H}}{Q}\right)^{\omega_{H}}\left(\frac{\mu_{J}}{Q \tau_{a}^{1 / j_{J}}}\right)^{2 j_{J} \omega_{J}}\left(\frac{\mu_{S}}{Q \tau_{a}}\right)^{\omega_{S}} H_{2}\left(Q^{2}, \mu_{H}\right) \\
& \times \widetilde{J}\left(\partial_{\Omega}+\ln \frac{\mu_{J}^{j_{J}}}{Q^{j_{J}} \tau_{a}}, \mu_{J}\right)^{2} \widetilde{S}\left(\partial_{\Omega}+\ln \frac{\mu_{S}}{Q \tau_{a}}, \mu_{S}\right) \frac{\exp \left(\gamma_{E} \Omega\right)}{\Gamma(1-\Omega)}
\end{aligned}
$$

The factorization scales $\mu_{i}$ are arbitrary scales that each function is evolved from, and the cross section is independent of the common scale $\mu$ that all functions are evolved to. If we kept the hard, jet, and soft functions exact to all orders, then the cross section would be independent of these factorization scales. Truncating the resummation at a given accuracy introduces dependence on the factorization scales due to the dropped (unknown) higherorder terms. Thus, by varying these scales a theoretical uncertainty due to the omitted higher-order terms can be estimated. ${ }^{2}$

It is worth remarking here that in integrating eq. (3.46) to obtain eq. (3.48) (or differentiating the latter to obtain the former) one assumes that the scales $\mu_{J, S}$ have not yet been chosen and are considered independent of $\tau_{a}$. Thus the derivative/integral does not act on them. This is represented by the top arrow in the commutative diagram in figure 2 . However, eventually, to minimize the $\operatorname{logs}$ of $\mu_{F} / Q \tau_{a}^{1 / j_{F}}$ in the jet and soft functions we will choose them to be functions of $\tau_{a}$. This is represented by the vertical arrows in figure 2 . If we do this first in eq. (3.46) or in eq. (3.48) before integrating/differentiating to obtain the other, then the latter operations are considerably more difficult and yield apparently different results at a truncated order of logarithmic accuracy. If all quantities in eqs. (3.46) and (3.48) are kept to all orders in $\alpha_{s}$, then either order of operations will yield exactly the same result - the cross section is independent of the scale choices $\mu_{F}$. However, at a

\footnotetext{
${ }^{2}$ Typically these scales are varied up and down by a factor of 2 . More reliable predictions and estimates of theoretical uncertainties can be obtained by using so-called "profile scales" whose functional form varies with $\tau_{a}$, see e.g. [38, $\left.76,78,79\right]$.
} 


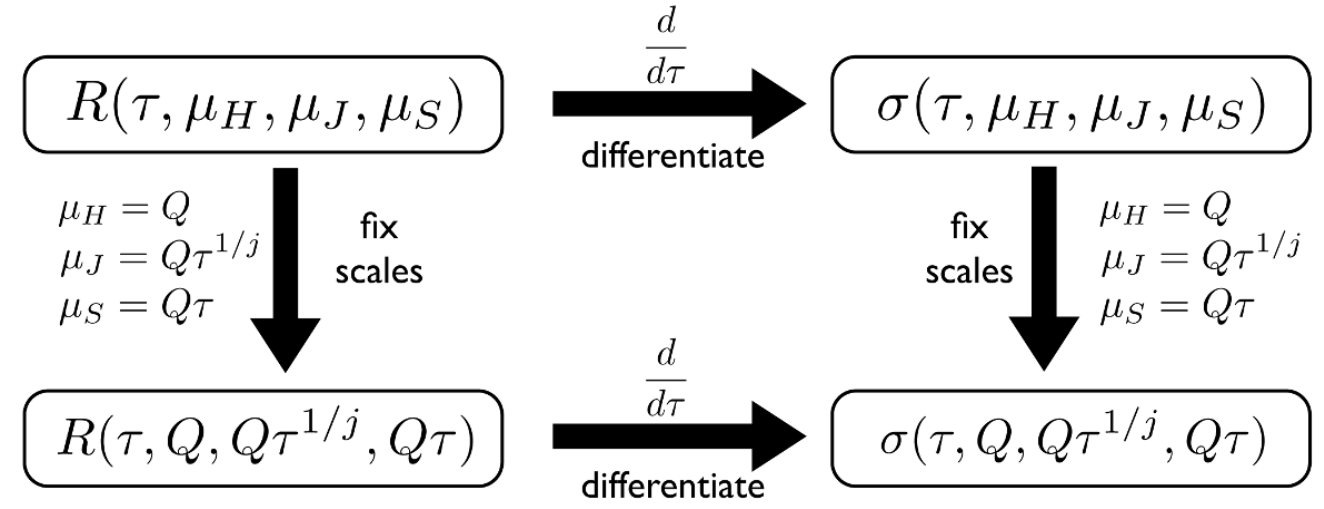

Figure 2. Commutative diagram for obtaining the resummed differential distribution from the resummed cumulant. The resummed distribution can be obtained by first differentiating the cumulant with free scales, and then choosing the scales to be $\tau$-dependent, such as the canonical choices eq. (3.22) indicated in the figure. Or, it can be obtained by first fixing the scales in the cumulant to be $\tau$-dependent, and then differentiating. Starting with expressions for $R$ truncated at a given $\mathrm{N}^{k} \mathrm{LL}$ order of accuracy, the latter method will produce more terms in $\sigma$ due to the derivative $d / d \tau$ seeing more $\tau$-dependent terms. However, if expressions for $R, \sigma$ are kept to all orders in $\alpha_{s}$, the two routes produce identical results.

truncated order, the two operations yield different results. We will remark on this further in section 4.3.1 discussing how to keep $\sigma$ and $R$ as closely to the same accuracy as possible when working to $\mathrm{N}^{k} \mathrm{LL}$ accuracy.

\subsection{Resummed event shape distributions in QCD}

The same 2-jet event shape distributions can be calculated directly in QCD, and such techniques were applied before SCET was developed [15]. One exploits the universal structure of soft and collinear singularities to write the resummed cross section. At next-to-leading logarithmic order, for example, the resummation is expressed in terms of an evolution kernel based on splitting functions. We will sketch here the derivation of the resummed angularity cross sections in direct QCD, and then move on to the final results one finds in the literature.

The analysis begins by determining the boost properties from the arguments of the soft and jet functions through which logarithmic behavior can occur. Then, each of the soft, jet, and hard functions obey two equations, one following from renormalization scale variation, the other from boost invariance. This pattern has been known for some time, and the general solutions derived in [16] are based on factorization and the strategy for resummations developed in [80]. In this case, the renormalization group equations are

$$
\begin{aligned}
\mu \frac{d}{d \mu} \ln S\left(\frac{Q}{\mu \nu}\left(\chi_{c}\right)^{a-1}, a, \alpha_{s}(\mu)\right) & =-\gamma_{s}\left(\alpha_{s}(\mu)\right), \\
\mu \frac{d}{d \mu} \ln J_{c}\left(\frac{Q \chi_{c}}{\mu}, \frac{Q}{\mu \nu}\left(\chi_{c}\right)^{a-1}, a, \alpha_{s}(\mu)\right) & =-\gamma_{J_{c}}\left(\alpha_{s}(\mu)\right), \\
\mu \frac{d}{d \mu} \ln H\left(\frac{Q}{\mu}, \frac{Q \chi_{c}}{\mu}, \alpha_{s}(\mu)\right) & =\gamma_{s}\left(\alpha_{s}(\mu)\right)+\sum_{c=1}^{2} \gamma_{J_{c}}\left(\alpha_{s}(\mu)\right),
\end{aligned}
$$


where

$$
\chi_{c}=\frac{Q}{2 p_{c} \cdot \hat{\xi}_{c}}
$$

with $\hat{\xi}_{c}$ the unit vector introduced in the definition of the QCD jet function, eq. (3.5), and $\beta_{c}$ a unit, lightlike vector in the direction of momentum $p_{c}^{\mu}=Q \beta_{c}^{\mu} / \sqrt{2}$ in the case of two-jet events. The anomalous dimensions can be defined to be scalars, because any dependence on the vectors $\xi_{c}$ or $\beta_{c}$ could be absorbed through a multiplicative redefinition of the hard function.

From the behavior under boosts, we derive an equation satisfied by the jets, which is the only one we need,

$$
\begin{aligned}
\frac{\partial}{\partial \ln \left(\chi_{c}\right)} \ln J_{c}( & \left.\frac{Q \chi_{c}}{\mu}, \frac{Q}{\mu \nu}\left(\chi_{c}\right)^{a-1}, a, \alpha_{s}(\mu)\right) \\
& =\mathcal{K}_{c}\left(\frac{Q}{\mu \nu}\left(\chi_{c}\right)^{a-1}, a, \alpha_{s}(\mu)\right)+\mathcal{G}_{c}\left(\frac{Q \chi_{c}}{\mu}, \alpha_{s}(\mu)\right),
\end{aligned}
$$

in terms of perturbative functions $\mathcal{K}$ and $\mathcal{G}$, whose low order expansions are given in [12]. Using these equations, we can evolve the soft function in the renormalization scale $\mu$, and the jet functions in $\mu$ and $\chi_{c}$ to organize all logarithms in terms of a limited set of perturbative functions, in much the same manner as for SCET. We will not give the details here, which for angularities are worked out in ref. [12], following [16]. The resulting moment-space distribution takes the form

$$
\widetilde{\sigma}\left(\nu_{a}\right)=\mathcal{N}(Q) \exp \left[E\left(\ln \nu_{a}\right)\right]
$$

where $\mathcal{N}(Q)$ is a log-free factor with an expansion in $\alpha_{s}$ with coefficients $C_{n}$ as in eq. (2.7),

$$
\mathcal{N}(Q)=1+\sum_{n=1}^{\infty}\left(\frac{\alpha_{s}(Q)}{4 \pi}\right)^{n} C_{n}
$$

which combines contributions from the hard, soft and jet functions evaluated at unit kinematic ratios at renormalization scale $Q$. The cumulant cross section is as usual written as the inverse Laplace transform of (3.54),

$$
R\left(\tau_{a}\right)=\mathcal{N}(Q) \frac{1}{2 \pi i} \int_{\mathcal{C}} \frac{d \nu_{a}}{\nu_{a}} \exp \left[\nu_{a} \tau_{a}+E\left(\ln \nu_{a}\right)\right]
$$

where $\mathcal{C}$ is the usual contour in the complex $\nu_{a}$ plane, to the right of singularities. The QCD exponent $E\left(\ln \nu_{a}\right)$ in eq. (3.54)) comes from fixed-order calculations of jet and soft functions that bear strong resemblance to those in SCET (although the organization of terms can differ), and is a combined object that describes the perturbative logarithms. It is built from fixed-order coefficients $A\left[\alpha_{s}\right], B\left[\alpha_{s}\right]$, where $A$ contains the cusp singularity contributions (and, at high orders, can contain additional terms) [12, 29, 30, 81]. The form 
of $E$ for the angularity distribution in eq. (3.56) in terms of $A$ and $B$ can be written

$$
\begin{aligned}
E\left(\ln \nu_{a}\right)= & 4 \int_{Q\left(e^{\gamma_{E}} \nu_{a}\right)^{-1 / j_{J}}}^{Q} \frac{d \mu^{\prime}}{\mu^{\prime}} A\left[\alpha_{s}\right] \ln \frac{\mu^{\prime}}{Q} \\
& -\frac{4}{1-a} \int_{\left.Q\left(e^{\gamma_{E}} \nu_{a}\right)^{-1 / j_{J}}\right)^{-1 / j_{S}}} \frac{d \mu^{\prime}}{\mu^{\prime}} A\left[\alpha_{s}\right] \ln \frac{\mu^{\prime}}{Q\left(e^{\gamma_{E}} \nu_{a}\right)^{-1 / j_{S}}} \\
& +2 \int_{Q\left(e^{\gamma_{E}} \nu_{a}\right)^{-1 / j_{J}}}^{Q} \frac{d \mu^{\prime}}{\mu^{\prime}} B_{J}\left[\alpha_{s}\right]+\int_{Q\left(e^{\gamma_{E}} \nu_{a}\right)^{-1 / j_{S}}}^{Q} \frac{d \mu^{\prime}}{\mu^{\prime}} B_{S}\left[\alpha_{s}\right] .
\end{aligned}
$$

where the integrals $B_{S}$ and $B_{J}$ generate non-leading singularities by evolving from the soft and jet scales respectively to the hard scale. In fact, it is always possible to absorb the term involving $B_{S}$ into the other two by following a method originally applied to threshold resummation in ref. [82]. We may use this freedom to define $A\left[\alpha_{s}\right]=\Gamma_{\text {cusp }}\left[\alpha_{s}\right]$, in which case one finds that $B_{S}\left[\alpha_{s}\right]$ begins only at order $\alpha_{s}^{2}$. In this way, in the QCD angularity analysis of [12], the three terms were reduced to two, to check consistency with the with the original CTTW result [15]. For the comparison to SCET that we will make in the next subsection, however, it is convenient to keep both of the $B$ terms.

To perform the inverse transform eq. (3.56), the exponent is expanded about $\ln \nu_{a}=$ $\ln \left(1 / \tau_{a}\right)[15]$

$$
E\left(\ln \nu_{a}\right)=\sum_{n=0}^{\infty} \frac{1}{n !} \ln ^{n}\left(\nu_{a} \tau_{a}\right)\left[\left.\frac{d^{n}}{d\left(\ln \nu_{a}\right)^{n}} E\left(\ln \nu_{a}\right)\right|_{\nu_{a}=1 / \tau_{a}}\right] .
$$

Defining $\bar{E}=E\left(\ln 1 / \tau_{a}\right)$ and the derivatives

$$
\left.\bar{E}^{\prime} \equiv \frac{d}{d \ln \nu_{a}} E\left(\ln \nu_{a}\right)\right|_{\nu_{a}=1 / \tau_{a}}, \ldots,\left.\bar{E}^{(n)} \equiv \frac{d^{n}}{d \ln \nu_{a}^{n}} E\left(\ln \nu_{a}\right)\right|_{\nu_{a}=1 / \tau_{a}}, \ldots
$$

we can write

$$
\begin{aligned}
\exp \left[E\left(\ln \nu_{a}\right)\right] & =\exp \left[\sum_{n=2}^{\infty} \frac{1}{n !} \bar{E}^{(n)} \partial_{\bar{E}^{\prime}}^{n}\right] \exp \left[\bar{E}+\bar{E}^{\prime} \ln \left(\nu_{a} \tau_{a}\right)\right] \\
& \equiv \widehat{T}\left(\bar{E}^{\prime}\right) \exp \left[\bar{E}+\bar{E}^{\prime} \ln \left(\nu_{a} \tau_{a}\right)\right]
\end{aligned}
$$

This uses the same kind of derivative operator as in the SCET resummation eq. (3.46), as $\bar{E}^{\prime}$ is a resummed series and derivatives with respect to it generate additional $\tau_{a}$ dependence. As in the SCET case, it allows us to perform the inverse transform eq. (3.56) analytically. The result is

$$
R\left(\tau_{a}\right)=\mathcal{N}(Q) \exp (\bar{E}) \widehat{T}\left(\bar{E}^{\prime}\right) \frac{1}{\Gamma\left(1-\bar{E}^{\prime}\right)}
$$

The operator $\widehat{T}$ contains an infinite series of terms (generalizing the result eq. (85) in [15]; a similar form for threshold resummation in Drell-Yan appeared in [77]). However, the series is well-ordered, as it is easy to see that the $\bar{E}^{(n)}$ are sequentially higher order $\left(\bar{E}^{(n)}\right.$ is $\mathrm{N}^{n} \mathrm{LL}$, counting in the resummed exponent). Therefore to work to a given order we simply 
truncate the series. As with SCET, since the function that the derivatives are acting on is known, the derivatives with respect to $\bar{E}^{\prime}$ represent a simple replacement rule.

Note that eq. (3.61) contains no free scales like $\mu_{H, J, S}$. As we will see below, they have implicitly been set to the values

$$
\mu_{H}=Q, \quad \mu_{J}=\bar{\mu}_{J} \equiv Q \bar{\tau}_{a}^{1 / j_{J}}, \quad \mu_{S}=\bar{\mu}_{S} \equiv Q \bar{\tau}_{a},
$$

where

$$
\bar{\tau}_{a} \equiv e^{-\gamma_{E}} \tau_{a}
$$

Thus eq. (3.61) sits in the lower left-hand box in figure 2 (except with $\mu_{J, S}$ rescaled to the values in eq. (3.62)). We will show below how to generalize it to have free scales $\mu_{H, J, S}$ as represented in the top left box in figure 2 .

Before comparing the cross sections in QCD and SCET, we note that, using the definitions in eq. (3.28), we can write the function $E\left(\ln \nu_{a}\right)$ as

$$
\begin{aligned}
E\left(\ln \nu_{a}\right)= & -2 j_{J} \kappa_{J} \int_{Q\left(e^{\left.\gamma_{E} \nu_{a}\right)^{-1 / j_{J}}}\right.}^{Q} \frac{d \mu^{\prime}}{\mu^{\prime}} A\left[\alpha_{s}\right] \ln \frac{\mu^{\prime}}{Q\left(e^{\gamma_{E}} \nu_{a}\right)^{-1 / j_{J}}} \\
& -j_{S} \kappa_{S} \int_{Q\left(e^{\left.\gamma_{E} \nu_{a}\right)^{-1 / j_{S}}}\right.}^{Q} \frac{d \mu^{\prime}}{\mu^{\prime}} A\left[\alpha_{s}\right] \ln \frac{\mu^{\prime}}{Q\left(e^{\gamma_{E}} \nu_{a}\right)^{-1 / j_{S}}} \\
& +2 \int_{Q\left(e^{\left.\gamma_{E} \nu_{a}\right)^{-1 / j_{J}}}\right.}^{Q} \frac{d \mu^{\prime}}{\mu^{\prime}} B_{J}\left[\alpha_{s}\right]+\int_{Q\left(e^{\gamma_{E}} \nu_{a}\right)^{-1 / j_{S}}}^{Q} \frac{d \mu^{\prime}}{\mu^{\prime}} B_{S}\left[\alpha_{s}\right] .
\end{aligned}
$$

This will make it easier to compare to the SCET resummed form.

\subsection{Equivalence between QCD and SCET resummation}

A natural question to ask is, do the SCET and QCD cross sections eqs. (3.48) and (3.61) return the same result for the same observable? One answer is that they had better when working to all orders, or someone is doing something wrong. But a more pragmatic (and important) question is, if we truncate to a given order in logarithm counting do the two methods still agree? This question has many possible answers. QCD and SCET may exactly agree, given some conventions for how to truncate each resummed series at a given order. They may agree on the terms up to the required accuracy but naturally include different sets of subleading terms. They may disagree at the requested level of accuracy (pointing to a fundamental problem in one of the methods). We will show, that written in suitable form, the two formalisms agree exactly. The process of this rewriting will illuminate and improve the results of both methods.

A detailed comparison between the two resummed cross sections above will only make these questions more pointed: the two results eqs. (3.48) and (3.61) look quite similar in form, and one might believe that appropriate relations between the QCD $A\left[\alpha_{s}\right]$ and $B_{i}\left[\alpha_{s}\right]$ functions and the SCET $\Gamma_{\text {cusp }}\left[\alpha_{s}\right]$ and $\gamma_{i}\left[\alpha_{s}\right]$ anomalous dimensions would put them in complete agreement. In fact, this is what we will find in this section. We start by building the basic correspondence between the two forms, showing that at all orders the two cross sections agree. Analogous but distinct considerations apply to the analysis of threshold 


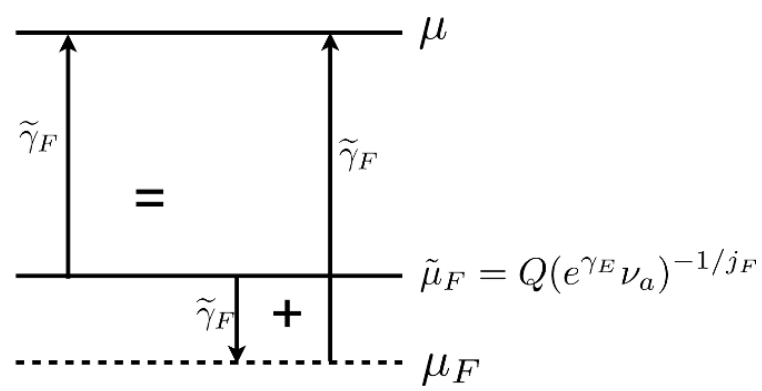

Figure 3. Evolution from the natural scale. In eq. (3.66), we express a function $\widetilde{F}\left(L_{F}(\mu), \mu\right)$ at the factorization scale $\mu$ by performing the RG evolution from its natural scale $\tilde{\mu}_{F}$ where all the large logs in the function are minimized. We further break this evolution into two pieces, one from the natural scale $\widetilde{\mu}_{F}$ to another arbitrary scale $\mu_{F}$, and from $\mu_{F}$ to $\mu$. We may choose to vary the arbitrary scale $\mu_{F}$ in order to estimate theoretical uncertainty in a prediction for $F$ at finite resummed accuracy.

resummation [29, 30, 32-34] when treated in moment space. In fact the correspondence between SCET and dQCD forms requires a more elaborate analysis for event shapes than for threshold resummation in moment space, but we will see that the resulting physical predictions for event shapes are analytically equivalent, in a way that has not been found for threshold resummed cross sections.

We will work mostly with the SCET formalism, primarily for the explicit dependence on free scales $\mu_{F}$ that they display. We will massage the SCET form into one easily relatable to the QCD cross section, in particular identifying the scale choices $\mu_{F}$ that make the SCET and QCD forms transparently equivalent. To be concrete, we begin first with the form of the resummed jet function in the two formalisms.

From eq. (3.37) or eq. (3.44) we already know how to express the jet (or soft) function at one scale $\mu$ by $\mathrm{RG}$ evolving from another scale $\mu_{J}\left(\mu_{S}\right)$. However, by performing a series of manipulations we can re-express eqs. (3.37) and (3.44) in a form that makes the connection with direct QCD in eq. (3.61) as transparent as possible and also exponentiates as many logs as possible. For instance, there are series of logs in the prefactor of eq. (3.44) in $\widetilde{F}\left(\partial_{\omega_{F}}, \mu_{0}\right)$, which are always truncated at some order in $\alpha_{s}$, generated by the action of the derivatives $\partial_{\omega_{F}}^{n}$ acting on the gamma function. We will put eq. (3.44) in a form that has as many of these logs explicitly exponentiated as possible, leaving the prefactor free of logs.

We start with the evolution from the natural scale for jet functions in transform space,

$$
\widetilde{\mu}_{J}=Q\left(e^{\gamma_{E}} \nu_{a}\right)^{-1 / j_{J}} .
$$

We then break the evolution into two pieces, that from $\widetilde{\mu}_{J}$ to $\mu_{J}$ and that from $\mu_{J}$ to $\mu$, illustrated in figure 3. Working from eqs. (3.37) and (3.43), we have,

$$
\widetilde{J}\left(L_{J}(\mu), \mu\right)=\widetilde{J}\left(0, \widetilde{\mu}_{J}\right) \exp \left(\int_{\tilde{\mu}_{J}}^{\mu_{J}} \frac{d \mu^{\prime}}{\mu^{\prime}} \widetilde{\gamma}_{J}\left(x_{a}, \mu^{\prime}\right)\right) \exp \left(\int_{\mu_{J}}^{\mu} \frac{d \mu^{\prime}}{\mu^{\prime}} \widetilde{\gamma}_{J}\left(x_{a}, \mu^{\prime}\right)\right),
$$

and similarly for the soft function, for which the discussion below would be entirely parallel. Note that $L_{J}\left(\widetilde{\mu}_{J}\right)=0$; hence the first argument of $\widetilde{J}$ on the right-hand side is 0 . 
Our goal is to express each piece in eq. (3.66) in terms of the variable scale $\mu_{J}$. The evolution from $\mu_{J}$ to $\mu$ is straightforward, in the notation of eqs. (3.37) and (3.42):

$$
\exp \left(\int_{\mu_{J}}^{\mu} \frac{d \mu^{\prime}}{\mu^{\prime}} \widetilde{\gamma}_{J}\left(x_{a}, \mu^{\prime}\right)\right)=\exp \left[K_{J}\left(\mu, \mu_{J}\right)\right] \exp \left[L_{J}\left(\mu_{J}\right) \omega_{J}\left(\mu, \mu_{J}\right)\right] .
$$

In dealing with the evolution from $\widetilde{\mu}_{J}$ to $\mu_{J}$, we will use derivatives with respect to $\omega_{J}$ to generate $L_{J}$ dependence. We express each scale in terms of $L_{J} \equiv L_{J}\left(\mu_{J}\right)$ and $L_{J}^{\prime} \equiv L_{J}\left(\mu^{\prime}\right)$

$$
\begin{aligned}
\tilde{\mu}_{J} & =\mu_{J} e^{-L_{J} / j_{J}}, \\
\mu^{\prime} & =\widetilde{\mu}_{J} e^{L_{J}^{\prime} / j_{J}}=\mu_{J} e^{\left(L_{J}^{\prime}-L_{J}\right) / j_{J}} .
\end{aligned}
$$

Changing variables from $\mu^{\prime}$ to $u=L_{J}^{\prime} / L_{J}$, the evolution from $\widetilde{\mu}_{J}$ to $\mu_{J}$ is

$$
\begin{array}{r}
\exp \left(\int_{\widetilde{\mu}_{J}}^{\mu_{J}} \frac{d \mu^{\prime}}{\mu^{\prime}} \widetilde{\gamma}_{J}\left(x_{a}, \mu^{\prime}\right)\right)=\exp \left(\int _ { 0 } ^ { 1 } d u \left\{-j_{J} \kappa_{J} \Gamma_{\text {cusp }}^{q}\left[\alpha_{s}\left(\mu e^{(u-1) L_{J} / j_{J}}\right)\right] \frac{L_{J}^{2}}{j_{J}^{2}} u\right.\right. \\
\left.\left.+\gamma_{J}\left[\alpha_{s}\left(\mu_{J} e^{(u-1) L_{J} / j_{J}}\right)\right] \frac{L_{J}}{j_{J}}\right\}\right) .
\end{array}
$$

This form is convenient to Taylor expand about $u=1$, that is, $\mu^{\prime}=\mu_{J}$, allowing us then to carry out the $u$ integral explicitly. After some analysis we obtain

$$
\begin{gathered}
-j_{J} \kappa_{J} \int_{0}^{1} d u \Gamma_{\text {cusp }}^{q}\left[\alpha_{s}\left(\mu_{J} e^{(u-1) L_{J} / j_{J}}\right)\right] \frac{L_{J}^{2}}{j_{J}^{2}} u \\
=-j_{J} \kappa_{J} \sum_{n=2}^{\infty} \frac{1}{n !}\left(-\frac{L_{J}}{j_{J}}\right)^{n} \frac{d^{n}}{d\left(\ln \mu_{J}\right)^{n}} \int_{\mu_{J}}^{\mu} \frac{d \mu^{\prime}}{\mu^{\prime}} \Gamma_{\mathrm{cusp}}\left[\alpha_{s}\left(\mu^{\prime}\right)\right] \ln \frac{\mu^{\prime}}{\mu_{J}} \\
\int_{0}^{1} d u \gamma_{J}\left[\alpha_{s}\left(\mu_{J} e^{(u-1) L_{J} / j_{J}}\right)\right] \frac{L_{J}}{j_{J}} \\
=\sum_{n=1}^{\infty} \frac{1}{n !}\left(-\frac{L_{J}}{j_{J}}\right)^{n} \frac{d^{n}}{d\left(\ln \mu_{J}\right)^{n}} \int_{\mu_{J}}^{\mu} \frac{d \mu^{\prime}}{\mu^{\prime}} \gamma_{J}\left[\alpha_{s}\left(\mu^{\prime}\right)\right]
\end{gathered}
$$

Additionally, one can show by Taylor expansion around $\widetilde{\mu}_{J}=\mu_{J}$,

$$
\begin{aligned}
\widetilde{J}\left(0, \widetilde{\mu}_{J}\right) & =\widetilde{J}(0, \mu) \frac{\widetilde{J}\left(0, \widetilde{\mu}_{J}\right)}{\widetilde{J}(0, \mu)} \\
& =\widetilde{J}(0, \mu) \exp \left\{\sum_{n=0}^{\infty} \frac{1}{n !}\left(-\frac{L_{J}}{j_{J}}\right)^{n} \frac{d^{n}}{d\left(\ln \mu_{J}\right)^{n}}\left[-\int_{\mu_{J}}^{\mu} \frac{d \mu^{\prime}}{\mu^{\prime}} \frac{d \ln \widetilde{J}\left(0, \mu^{\prime}\right)}{d \ln \mu^{\prime}}\right]\right\} .
\end{aligned}
$$

If we put the various pieces of the evolution together, we obtain the jet function in eq. (3.66) at arbitrary $\mu$,

$$
\begin{aligned}
\widetilde{J}\left(L_{J}(\mu), \mu\right)=\widetilde{J}(0, \mu) \exp \left[E_{J}\left(\mu, \mu_{J}\right)+\sum_{n=2}^{\infty} \frac{1}{n !}\left(-\frac{L_{J}}{j_{J}}\right)^{n} \frac{d^{n} E_{J}\left(\mu, \mu_{J}\right)}{d\left(\ln \mu_{J}\right)^{n}}\right] \times \\
\times \exp \left[-\frac{L_{J}}{j_{J}} \frac{d E_{J}\left(\mu, \mu_{J}\right)}{d \ln \mu_{J}}\right],
\end{aligned}
$$


where we have defined

$$
E_{J}\left(\mu, \mu_{J}\right) \equiv K_{J}\left(\mu, \mu_{J}\right)-\int_{\mu_{J}}^{\mu} \frac{d \mu^{\prime}}{\mu^{\prime}} \frac{d \ln \widetilde{J}\left(0, \mu^{\prime}\right)}{d \ln \mu^{\prime}} .
$$

Note that the second term, and thus the difference between $E_{J}$ and $K_{J}$, begins at $\mathcal{O}\left(\alpha_{s}^{2} \ln \left(\mu_{J} / \mu\right)\right)$, which is NNLL in table 1 . Defining the derivatives

$$
E_{J}^{\prime}\left(\mu, \mu_{J}\right)=\frac{d E_{J}\left(\mu, \mu_{J}\right)}{d \ln \mu_{J}}, \quad E_{J}^{(n)}\left(\mu_{J}\right)=\frac{d^{n} E_{J}\left(\mu, \mu_{J}\right)}{d\left(\ln \mu_{J}\right)^{n}}
$$

(note that taking two or more derivatives of $E_{J}$ with respect to $\mu_{J}$ removes its dependence on $\mu$ ) we find that eq. (3.72) can be expressed compactly as

$$
\widetilde{J}\left(L_{J}(\mu), \mu\right)=\widetilde{J}(0, \mu) \exp \left[E_{J}\left(\mu, \mu_{J}\right)+\sum_{n=2}^{\infty} \frac{1}{n !} E_{J}^{(n)}\left(\mu_{J}\right) \partial_{E_{J}^{\prime}}^{n}\right] \exp \left[-\frac{L_{J}\left(\mu_{J}\right)}{j_{J}} E_{J}^{\prime}\left(\mu, \mu_{J}\right)\right] .
$$

Now, recalling that $L_{J}\left(\mu_{J}\right)=\ln \left(\mu_{J}^{j_{J}} e^{\gamma_{E}} x_{a}\right)$, we can inverse Laplace transform eq. (3.75) from $x_{a}$ back to $t_{a}$ and obtain in momentum space:

$$
J\left(t_{a}, \mu\right)=\widetilde{J}(0, \mu) \exp \left[E_{J}\left(\mu, \mu_{J}\right)+\sum_{n=2}^{\infty} \frac{1}{n !} E_{J}^{(n)}\left(\mu_{J}\right) \partial_{E_{J}^{\prime}}^{n}\right] \frac{1}{t_{a}}\left(\frac{\mu_{J}^{j_{J}} e^{\gamma_{E}}}{t_{a}}\right)^{-E_{J}^{\prime}\left(\mu, \mu_{J}\right) / j_{J}} \frac{1}{\Gamma\left(E_{J}^{\prime} / j_{J}\right)} .
$$

We have succeeded in expressing $\widetilde{J}\left(L_{J}(\mu), \mu\right)$ or $J\left(t_{a}, \mu\right)$ at one scale $\mu$ in terms of RG evolution from any other scale $\mu_{J}$, with no explicit logs left over in the factor $\widetilde{J}(0, \mu)$, which must be truncated at fixed order in $\alpha_{s}$ in eqs. (3.75) and (3.76). They are all contained in the exponentiated evolution kernels. (The exponentiated derivative operators must, however, still be truncated at some fixed order in practice; still, the exponentiated form gives an easy prescription that can be evaluated to any desired order.)

Similarly for the soft function, by analogy to eq. (3.76)

$$
S(k, \mu)=\widetilde{S}(0, \mu) \exp \left[E_{S}\left(\mu, \mu_{S}\right)+\sum_{n=2}^{\infty} \frac{1}{n !} E_{S}^{(n)}\left(\mu_{S}\right) \partial_{E_{S}^{\prime}}^{n}\right] \frac{1}{k}\left(\frac{\mu_{S} e^{\gamma_{E}}}{k}\right)^{-E_{S}^{\prime}\left(\mu, \mu_{S}\right)} \frac{1}{\Gamma\left(E_{S}^{\prime}\right)},
$$

where

$$
\begin{aligned}
& E_{S}\left(\mu, \mu_{S}\right) \equiv K_{S}\left(\mu, \mu_{S}\right)-\int_{\mu_{S}}^{\mu} \frac{d \mu^{\prime}}{\mu^{\prime}} \frac{d \ln \widetilde{S}\left(0, \mu^{\prime}\right)}{d \ln \mu^{\prime}}, \\
& E_{S}^{\prime}\left(\mu, \mu_{S}\right)=\frac{d E_{S}\left(\mu, \mu_{S}\right)}{d \ln \mu_{S}}, \quad E_{S}^{(n)}\left(\mu_{S}\right)=\frac{d^{n} E_{S}\left(\mu, \mu_{S}\right)}{d\left(\ln \mu_{S}\right)^{n}} .
\end{aligned}
$$

Finally, we can obtain the total cross section by starting with the Laplace-transformed factorization theorem, obtained by transforming eq. (3.2):

$$
\widetilde{\sigma}(\nu, \mu)=\int_{0}^{\infty} d \tau_{a} e^{-\nu \tau_{a}} \frac{1}{\sigma_{0}} \frac{d \sigma}{d \tau_{a}}=H_{2}\left(Q^{2}, \mu\right) \widetilde{J}\left(L_{J}(\mu), \mu\right)^{2} \widetilde{S}\left(L_{S}(\mu), \mu\right),
$$


plugging in the form eq. (3.72) of the jet function and similarly for the soft function, Laplace transforming all at once from $\nu$ back to $\tau_{a}$, and integrating over $\tau_{a}$ to obtain the cumulant:

$$
\begin{aligned}
R\left(\tau_{a}\right)= & H_{2}\left(Q^{2}, \mu_{H}\right)\left(\frac{\mu_{H}}{Q}\right)^{\omega_{H}\left(\mu, \mu_{H}\right)} e^{K_{H}\left(\mu, \mu_{H}\right)} \widetilde{J}(0, \mu)^{2} \widetilde{S}(0, \mu) \\
& \times \exp \left[2 E_{J}\left(\mu, \mu_{J}\right)+E_{S}\left(\mu, \mu_{S}\right)\right] \exp \left[\sum_{n=2}^{\infty} \frac{1}{n !}\left(2 E_{J}^{(n)}\left(\mu_{J}\right) \partial_{2 E_{J}^{\prime}}^{n}+E_{S}^{(n)}\left(\mu_{S}\right) \partial_{E_{S}^{\prime}}^{n}\right)\right] \\
& \times\left(\frac{Q^{j_{J}} \tau_{a}}{\mu_{J}^{j_{J}} e^{\gamma_{E}}}\right)^{2 E_{J}^{\prime}\left(\mu, \mu_{J}\right) / j_{J}}\left(\frac{Q \tau_{a}}{\mu_{S} e^{\gamma_{E}}}\right)^{E_{S}^{\prime}\left(\mu, \mu_{S}\right)} \frac{1}{\Gamma\left(1+2 E_{J}^{\prime} / j_{j}+E_{S}^{\prime}\right)}
\end{aligned}
$$

This generalizes the NLL result eq. (85) of [15] and those of $[12,13]$ to arbitrarily high accuracy and restores its dependence on variable hard, jet and soft scales $\mu_{H, J, S}$.

We can show that the SCET cross section now written in the form eq. (3.80) and the QCD cross section eq. (3.61) are identical if some identifications and particular scale choices are made. In eq. (3.80), let us choose to run the hard, jet, and soft functions from the scales

$$
\mu_{H}=Q, \quad \bar{\mu}_{J}=Q \bar{\tau}_{a}^{1 / j_{J}}, \quad \bar{\mu}_{S}=Q \bar{\tau}_{a},
$$

where $\bar{\tau}_{a}=e^{-\gamma_{E}} \tau_{a}$ as defined in eq. (3.63), to the scale $\mu=Q$. Then we have the expression [77]

$$
R\left(\tau_{a}\right)=H_{2}\left(Q^{2}, Q\right) \widetilde{J}(0, Q)^{2} \widetilde{S}(0, Q) \exp \left[\bar{E}+\sum_{n=2}^{\infty} \frac{1}{n !} \bar{E}^{(n)} \partial_{\bar{E}^{\prime}}^{n}\right] \frac{1}{\Gamma\left(1-\bar{E}^{\prime}\right)},
$$

with

$$
\begin{aligned}
\bar{E} & \equiv 2 \bar{E}_{J}+\bar{E}_{S} \\
\bar{E}^{\prime} & \equiv \frac{d \bar{E}}{d \ln \left(1 / \tau_{a}\right)} \\
\bar{E}^{(n)} & \equiv \frac{d^{n} \bar{E}}{d\left(\ln \left(1 / \tau_{a}\right)\right)^{n}}
\end{aligned}
$$

where $\bar{E}_{J, S} \equiv E_{J, S}\left(Q, \bar{\mu}_{J, S}\right)$. Note that the derivatives in eq. (3.83) are now with respect to $\ln \left(1 / \tau_{a}\right)$ instead of $\ln \mu_{J, S}$ as in eq. (3.74).

Remarkably, the SCET cross section in eq. (3.82) is now exactly in the form of the QCD cross section eq. (3.61). They are equal if we identify

$$
\begin{aligned}
A\left[\alpha_{s}\right] & =\Gamma_{\text {cusp }}\left[\alpha_{s}\right], \quad B_{F}\left[\alpha_{s}\right]=\gamma_{F}\left[\alpha_{s}\right]-\frac{d \ln \widetilde{F}\left(0, \mu_{F}\right)}{d \ln \mu_{F}}, \\
\mathcal{N}(Q) & =H\left(Q^{2}, Q\right) \widetilde{J}(0, Q)^{2} \widetilde{S}(0, Q) .
\end{aligned}
$$

Similar identifications were found in ref. [29, 30, 32-34] for threshold resummation in DrellYan production.

The identification is not without significance for direct QCD: with these identifications, eq. (3.80) also serves to generalize the usual QCD direct resummation formulae by 
endowing them with free scales $\mu, \mu_{H, J, S}$ and thus the flexibility to vary these scales away from the values in eq. (3.81). This allows one to match the resummation onto fixed-order perturbation theory using profile scales for $\mu_{J, S}$ to smoothly interpolate between the resummation and fixed-order regimes, and in general allows one to obtain better estimates of theoretical uncertainty.

We note that in eq. (3.82),

$$
\bar{E}^{\prime}=\bar{\Omega}+\frac{2 B_{J}\left[\alpha_{s}\left(\bar{\mu}_{J}\right)\right]}{j_{J}}+B_{S}\left[\alpha_{s}\left(\bar{\mu}_{S}\right)\right]
$$

where $\bar{\Omega}=2 \omega_{J}\left(Q, \bar{\mu}_{J}\right)+\omega_{S}\left(Q, \bar{\mu}_{S}\right)$. This looks like the argument of the gamma function in the form of the SCET distribution shown in eq. (3.46), shifted by $2 B_{J} / j_{J}+B_{S}$. This shift is the result of resumming many of the $\partial_{\Omega}$-dependent terms in the jet and soft operators in eq. (3.46), which is effectively what has been done by resumming all the $\mu_{J}$-dependent terms in the jet function into the exponential factors in eq. (3.75), and similarly for the soft function. Also, note that the choices eq. (3.81) for jet and soft scales differ from the usual choices in SCET by rescalings of $\tau_{a}$ to $\bar{\tau}_{a} \equiv e^{-\gamma_{E}} \tau_{a}$. This just causes the factor of $e^{\gamma_{E} \Omega}$ in the SCET resummed distribution eq. (3.46) to be absorbed into $K_{J, S}$. Of course, the different scale choices lead to equivalent results at a given order of accuracy.

Finally, we note that the resummed form in eq. (3.82) with the non-cusp and $\beta$ function terms resummed into the gamma function is, to our knowledge, new for SCET. Thus it formally resums more terms to all orders in $\alpha_{s}$ than most previous formulae in the SCET literature, capturing more of the non-cusp anomalous dimension and beta functiondependent terms to all orders in $\alpha_{s}$, making it more accurate in the sense of maintaining as closely as possible its equivalence to the inverse Laplace transform of $\widetilde{\sigma}(\nu)$.

\subsection{Interlude}

In section 3.4 we obtained the central result of the paper, eq. (3.82) which puts the SCET resummed cross section into a form transparently equivalent to the dQCD form eq. (3.61). Before moving on, let us take stock of what we have found, and what is yet to come.

- Eq. (3.80) is a rewriting of the usual form of the SCET cumulant cross section eq. (3.48). Making the scale choices eq. (3.81) in the SCET form eq. (3.80) yields the form eq. (3.82), which is transparently equivalent to the dQCD form eq. (3.61). Eq. (3.73) for $E_{J}$ and eq. (3.78) for $E_{S}$, along with the definitions eq. (3.83) and identifications eq. (3.84) complete the dictionary for this equivalence.

- The form eq. (3.80) prior to fixing scales $\mu_{H, J, S}$ shows how to restore the dependence on these scales in the dQCD cross section eq. (3.61), in which the scales are implicitly fixed to the values in eq. (3.62). This is to our knowledge the first time the final result for the momentum-space distribution has been written with variable scales in dQCD notation, and with all derivative terms $n \geq 2$ written explicitly.

- The forms eqs. (3.80) and (3.82) differ from the typical SCET form eq. (3.48) due to the additional non-cusp anomalous dimension and beta function dependent terms 
that are included in $E_{J, S}$ in eqs. (3.73) and (3.78) and are now all in the exponent or argument of the gamma function in eqs. (3.80) and (3.82). ${ }^{3}$ The prefactors in eq. (3.82) are free of logs, and can be truncated at fixed-order without losing factors containing $\operatorname{logs}$ of $\tau_{a}$. While the extra terms are formally subleading, they help maintain closer equivalence to the Laplace transform to higher orders in $\alpha_{s}$.

Now there remains the question of how to evaluate each piece of eq. (3.82) to achieve $\mathrm{N}^{k} \mathrm{LL}$ or $\mathrm{N}^{k} \mathrm{LL}^{\prime}$ resummed accuracy. We will show in section 4 :

- It is easiest to define resummed accuracy in terms of the Laplace transform eq. (2.8) since it exponentiates simply in terms of the exponent $\bar{E}$ for which a straightforward prescription can be given for its computation. We will adopt the common definition of $\mathrm{N}^{k} \mathrm{LL}$ accuracy in terms of the order to which $\bar{E}$ is computed in eq. (2.8).

- We will define prescriptions for computing the cumulant $R(\tau)$ or distribution $\sigma(\tau)$ to $\mathrm{N}^{k} \mathrm{LL}$ accuracy so that they remain at least as accurate as $\widetilde{\sigma}(\nu)$ computed to $\mathrm{N}^{k} \mathrm{LL}$ accuracy.

- For $R(\tau)$ these prescriptions make it preferable to keep at least the $n=2$ term in the sum of derivative operators in the exponent of eq. (3.82) at NLL accuracy, $n=3$ at NNLL, etc. The exponential of these operators can itself be truncated at $\mathcal{O}\left(\alpha_{s}\right)$ at NLL, $\mathcal{O}\left(\alpha_{s}^{2}\right)$ at NNLL, etc. This is one higher order than is often kept. We will show why this prescription does better in maintaining equivalent accuracy with the Laplace transform $\widetilde{\sigma}(\nu)$. In the standard SCET form eq. (3.48), the appropriate prescription requires keeping the differential operator terms in $\widetilde{J}, \widetilde{S}$ to at least $\mathcal{O}\left(\alpha_{s}\right)$ for NLL, $\mathcal{O}\left(\alpha_{s}^{2}\right)$ for NNLL, etc.

- For $\sigma(\tau)$, we will show that the two procedures of computation, 1) directly from eq. (3.46) and then fixing scales $\mu_{F}$, or 2 ) by differentiating $R(\tau)$ in eq. (3.48) after fixing scales $\mu_{F}$, may give different answers when standard rules for computing to $\mathrm{N}^{k} \mathrm{LL}$ accuracy are applied. To ensure that procedure 1 gives as accurate an answer as procedure 2, we will show that the differential operator terms in $\widetilde{J}, \widetilde{S}$ must not be truncated according to standard rules too soon, namely when $\sigma(\tau)$ is still written in the form eq. (3.46). Instead, we will show it is expedient to pull a factor of $-\Omega$ through the $\widetilde{J}, \widetilde{S}$ operators in eq. (3.46) first, and then keep a subset of the extra terms generated by the action of the $\partial_{\Omega}$ derivatives on this factor. At $\mathrm{N}^{k} \mathrm{LL}^{\prime}$ order, though, this is not strictly necessary.

The reader who wishes to skip to the final results for formulae for the Laplace transform $\widetilde{\sigma}(\nu)$, cumulant $R(\tau)$, and differential distribution $\sigma(\tau)$, and the prescriptions for how to evaluate each properly to $\mathrm{N}^{k} \mathrm{LL}$ or $\mathrm{N}^{k} \mathrm{LL}^{\prime}$ accuracy can now turn to section 5 . First, in section 4 , we will develop and justify these prescriptions in detail.

\footnotetext{
${ }^{3} \mathrm{~A}$ similar exponentiated form for the momentum-space SCET soft function for $t \bar{t}$ production in hadron collisions was also found in [83]. Also, refs. [32,33] observed in the context of threshold resummation that inclusion of more terms in the SCET exponent as we did in eqs. (3.73) and (3.78) would bring SCET and dQCD forms into closer agreement. Our formulae generalize these observations to all orders for the case of event shape distributions.
} 


\section{Achieving $\mathrm{N}^{k} \mathrm{LL}$ logarithmic accuracy}

In eq. (2.6) we defined what $\mathrm{N}^{k} \mathrm{LL}$ accuracy means, in terms of the number of logs in the exponent of the Laplace transform $\widetilde{\sigma}(\nu)$ that are known. In this section, we consider how to actually achieve $N^{k} \mathrm{LL}$ accuracy in practice. We review the procedure for the Laplace transform of the distribution $\widetilde{\sigma}(\nu)$, and the consider how to compute the cumulant $R(\tau)$ or differential event shape distribution $\sigma(\tau)$ to the equivalent accuracy. The formalism reviewed in the previous section allows this to be done systematically by calculating anomalous dimensions $\gamma_{F}$ and hard, jet, and soft functions $H, J_{n, \bar{n}}, S$ order by order in $\alpha_{s}$. We will clarify to what order in $\alpha_{s}$ each of these quantities must be computed to achieve a given order of logarithmic accuracy. The procedure is standard and straightforward for $\tilde{\sigma}(\nu)$. It will turn out that usage of standard formulae for $R(\tau)$ and especially $\sigma(\tau)$ require special care in how they are used (namely, how different parts of them are truncated to finite order in $\alpha_{s}$ ) to preserve the same level of accuracy as $\widetilde{\sigma}(\nu)$. We will explain how to do this properly, and clarify some confusion that can easily arise from a casual reading of the existing literature.

Our aim in this section is to be rather pedagogical and give as clear explanations as possible for the prescriptions we present for evaluating $\widetilde{\sigma}(\nu), R(\tau)$, and $\sigma(\tau)$ to $\mathrm{N}^{k} \mathrm{LL}$ or $\mathrm{N}^{k} \mathrm{LL}^{\prime}$ accuracy. The level of detail is, accordingly, quite high. As we mentioned above, the reader who wishes to skip over it to reach the final results should turn directly to section 5 .

\subsection{Laplace transform}

The RG-evolved Laplace-transformed cross section $\widetilde{\sigma}(\nu)$ obtained from the factorization theorem eq. (3.3) by using the methods in section 3.2.2 takes the explicit form given in eq. (3.45),

$$
\begin{aligned}
\widetilde{\sigma}(\nu)= & e^{K} H\left(L_{H}, \mu_{H}\right) \widetilde{J}\left(L_{J}, \mu_{J}\right)^{2} \widetilde{S}\left(L_{S}, \mu_{S}\right) \\
& \times\left(\frac{\mu_{H}}{Q}\right)^{\omega_{H}\left(\mu, \mu_{H}\right)}\left(\frac{\mu_{J}\left(e^{\gamma_{E}} \nu\right)^{1 / j_{J}}}{Q}\right)^{2 j_{J} \omega_{J}\left(\mu, \mu_{J}\right)}\left(\frac{\mu_{S} e^{\gamma_{E}} \nu}{Q}\right)^{\omega_{S}\left(\mu, \mu_{S}\right)},
\end{aligned}
$$

or, expressing the logs in the jet and soft functions as the result of derivatives,

$$
\begin{aligned}
\widetilde{\sigma}(\nu)= & e^{K} H\left(L_{H}, \mu_{H}\right)\left(\frac{\mu_{H}}{Q}\right)^{\omega_{H}\left(\mu, \mu_{H}\right)}\left(\frac{\mu_{J} \nu^{1 / j_{J}}}{Q}\right)^{2 j_{J} \omega_{J}\left(\mu, \mu_{J}\right)}\left(\frac{\mu_{S} \nu}{Q}\right)^{\omega_{S}\left(\mu, \mu_{S}\right)-\Omega} \\
& \times \widetilde{J}\left(\partial_{\Omega}+\ln \frac{\mu_{J}^{j_{J}}}{Q^{j_{J}-1} \mu_{S}}, \mu_{J}\right)^{2} \widetilde{S}\left(\partial_{\Omega}, \mu_{S}\right)\left(\frac{\mu_{S} e^{\gamma_{E}} \nu}{Q}\right)^{\Omega} \cdot
\end{aligned}
$$

This form will be easier to inverse Laplace transform to the cumulant or distribution in $\tau_{a}$. In eqs. (4.1) and (4.2), we have defined the combined RG evolution kernels,

$$
\begin{aligned}
K & \equiv K_{H}\left(\mu, \mu_{H}\right)+2 K_{J}\left(\mu, \mu_{J}\right)+K_{S}\left(\mu, \mu_{S}\right) \\
\Omega & \equiv 2 \omega_{J}\left(\mu, \mu_{J}\right)+\omega_{S}\left(\mu, \mu_{S}\right),
\end{aligned}
$$


with the functions $K_{F}$ and $\omega_{F}$ defined in eq. (3.36). The hard, jet, and soft functions have fixed-order expansions of the form

$$
F\left(L_{F}, \mu_{F}\right)=\sum_{n=0}^{\infty}\left(\frac{\alpha_{s}\left(\mu_{F}\right)}{4 \pi}\right)^{n} F_{n}\left(L_{F}, \mu_{F}\right)
$$

where $F=H, \widetilde{J}, \widetilde{S}$, and to order $\alpha_{s}^{2}$ the coefficients $F_{n}$ are given by, using the evolution of the functions $F$ in eqs. (3.32) and (3.33),

$$
\begin{aligned}
F_{0}= & 1 \\
F_{1}= & \frac{\Gamma_{F}^{0}}{j_{F}^{2}} L_{F}^{2}+\frac{\gamma_{F}^{0}}{j_{F}} L_{F}+c_{F}^{1} \\
F_{2}= & \frac{1}{2 j_{F}^{4}}\left(\Gamma_{F}^{0}\right)^{2} L_{F}^{4}+\frac{\Gamma_{F}^{0}}{j_{F}^{3}}\left(\gamma_{F}^{0}+\frac{2}{3} \beta_{0}\right) L_{F}^{3} \\
& +\frac{1}{j_{F}^{2}}\left(\Gamma_{F}^{1}+\frac{1}{2}\left(\gamma_{F}^{0}\right)^{2}+\gamma_{F}^{0} \beta_{0}+c_{F}^{1} \Gamma_{F}^{0}\right) L_{F}^{2}+\frac{1}{j_{F}}\left(\gamma_{F}^{1}+c_{F}^{1} \gamma_{F}^{0}+2 c_{F}^{1} \beta_{0}\right) L_{F}+c_{F}^{2},
\end{aligned}
$$

where for $F=H, L_{H} \equiv \ln \left(\mu_{H} / Q\right)$ and for $F=\widetilde{J}, \widetilde{S}, L_{F}=\ln \left(\mu_{F}^{j_{F}} e^{\gamma_{E}} \nu / Q^{j_{F}}\right)$. In eq. (4.2), for the jet and soft functions, each $L_{F}$ gets replaced by the differential operator shown in the argument of $\widetilde{J}, \widetilde{S}$. Recall $j_{J}=2-a$ while $j_{H}=j_{S}=1$. The quantities $\Gamma_{F}^{n}$ are the coefficients in the expansion in $\alpha_{s}$ of $\Gamma_{F}$ in eq. (3.29),

$$
\Gamma_{F}\left[\alpha_{s}\left(\mu_{F}\right)\right]=\sum_{n=0}^{\infty}\left(\frac{\alpha_{s}\left(\mu_{F}\right)}{4 \pi}\right)^{n} \Gamma_{F}^{n},
$$

and similarly for $\gamma_{F}$ in eq. (3.30).

The expression eq. (4.2) for $\widetilde{\sigma}(\nu)$ contains logarithms of $\mu_{H} / Q$ and $\mu_{F}\left(e^{\gamma_{E}} \nu\right)^{1 / j_{F}} / Q$, which can be minimized in $\nu$ space by the choices of scales

$$
\mu=\mu_{H}=Q, \quad \mu_{F}=\widetilde{\mu}_{F} \equiv Q\left(e^{\gamma_{E}} \nu\right)^{-1 / j_{F}}
$$

Then $\widetilde{\sigma}(\nu)$ takes the particularly simple form,

$$
\widetilde{\sigma}(\nu)=H \widetilde{J}^{2} \widetilde{S} e^{K},
$$

where $F=H, \widetilde{J}, \widetilde{S}$ now have the simple log-free expansions

$$
F=1+\frac{\alpha_{s}\left(\widetilde{\mu}_{F}\right)}{4 \pi} c_{F}^{1}+\left(\frac{\alpha_{s}\left(\widetilde{\mu}_{F}\right)}{4 \pi}\right)^{2} c_{F}^{2}+\cdots
$$

eq. (4.8) organizes terms in $\widetilde{\sigma}(\nu)$ into a form much like the CTTW form of the radiator eq. (2.10). The exponent $K$ with the scale choices eq. (4.7) reduces to

$$
K=2 K_{J}\left(Q, Q s^{1 / j_{J}}\right)+K_{S}(Q, Q s),
$$

where $s \equiv e^{\gamma_{E}} \nu$. 


\begin{tabular}{|c|c|c|c|c|}
\hline$K_{n m}$ & $m=n+1$ & $m=n$ & $m=n-1$ & $m=n-2$ \\
\hline$n=1$ & $\frac{1}{2} \Gamma_{0}$ & & & \\
\hline$n=2$ & $\frac{1}{3} \Gamma_{0} \beta_{0}$ & $\frac{1}{2} \Gamma_{1}$ & & \\
\hline$n=3$ & $\frac{1}{3} \Gamma_{0} \beta_{0}^{2}$ & $\left(\Gamma_{0} \beta_{1}+2 \Gamma_{1} \beta_{0}\right)$ & $\frac{1}{2} \Gamma_{2}$ & \\
\hline$\vdots$ & $\vdots$ & $\vdots$ & $\vdots$ & $\vdots$ \\
\hline accuracy & LL & NLL & NNLL & $\mathrm{N}^{3} \mathrm{LL}$ \\
\hline
\end{tabular}

Table 3. Coefficients $K_{n m}$ in the expansion eq. (4.11) $K_{\Gamma}=\sum_{n, m} K_{n m}\left(\alpha_{s} / 4 \pi\right)^{n} \ln ^{m} s^{1 / j_{F}}$ up to $n=3$. A closed form of $K_{\Gamma}$ to $\mathrm{N}^{3} \mathrm{LL}$ accuracy is given in eq. (4.20).

\begin{tabular}{|c|c|c|c|}
\hline$k_{n m}$ & $m=n$ & $m=n-1$ & $m=n-2$ \\
\hline$n=1$ & $\gamma_{F}^{0}$ & & \\
\hline$n=2$ & $\gamma_{F}^{0} \beta_{0}$ & $\gamma_{F}^{1}$ & \\
\hline$n=3$ & $\frac{4}{3} \gamma_{F}^{0} \beta_{0}^{2}$ & $\gamma_{F}^{0} \beta_{1}+2 \gamma_{F}^{1} \beta_{0}$ & $\gamma_{F}^{2}$ \\
\hline$\vdots$ & $\vdots$ & $\vdots$ & $\vdots$ \\
\hline accuracy & NLL & NNLL & $\mathrm{N}^{3} \mathrm{LL}$ \\
\hline
\end{tabular}

\begin{tabular}{|c|c|c|c|}
\hline$\eta_{n m}$ & $m=n$ & $m=n-1$ & $m=n-2$ \\
\hline$n=1$ & $\Gamma_{0}$ & & \\
\hline$n=2$ & $\Gamma_{0} \beta_{0}$ & $\Gamma_{1}$ & \\
\hline$n=3$ & $\frac{4}{3} \Gamma_{0} \beta_{0}^{2}$ & $\Gamma_{0} \beta_{1}+2 \Gamma_{1} \beta_{0}$ & $\Gamma_{2}$ \\
\hline$\vdots$ & $\vdots$ & $\vdots$ & $\vdots$ \\
\hline accuracy & LL & NLL & NNLL \\
\hline
\end{tabular}

Table 4. Coefficients $k_{n m}$ and $\eta_{n m}$ in the expansions eq. (4.12) for $K_{\gamma_{F}}=$ $\sum_{n, m} k_{n m}\left(\alpha_{s} / 4 \pi\right)^{n} \ln ^{m} s^{1 / j_{F}}$ and eq. (4.13) for $\eta_{\Gamma}=\sum_{n, m} \eta_{n m}\left(\alpha_{s} / 4 \pi\right)^{n} \ln ^{m} s^{1 / j_{F}}$, up to $n=3$. Closed forms for $K_{\gamma_{F}}$ and $\eta_{\Gamma}$ up to $\mathrm{N}^{3} \mathrm{LL}$ accuracy are given by eqs. (4.22) and (4.23).

Each function $K_{F}$ above is given by eq. (3.36) in terms of $K_{\Gamma}$ and $K_{\gamma_{F}}$, whose fixedorder expansions take the form determined by the integrals in eq. (3.34),

$$
\begin{aligned}
K_{\Gamma}\left(Q, Q / s^{1 / j_{F}}\right)= & \left(\frac{\alpha_{s}(Q)}{4 \pi}\right)\left(K_{12} \ln ^{2} s^{1 / j_{F}}+K_{11} \ln s^{1 / j_{F}}\right) \\
& +\left(\frac{\alpha_{s}(Q)}{4 \pi}\right)^{2}\left(K_{23} \ln ^{3} s^{1 / j_{F}}+K_{22} \ln ^{2} s^{1 / j_{F}}+K_{21} \ln s^{1 / j_{F}}\right) \\
& +\left(\frac{\alpha_{s}(Q)}{4 \pi}\right)^{3}\left(K_{34} \ln ^{4} s^{1 / j_{F}}+K_{33} \ln ^{3} s^{1 / j_{F}}+K_{32} \ln ^{2} s^{1 / j_{F}}+K_{31} \ln s^{1 / j_{F}}\right) \\
& +\cdots
\end{aligned}
$$

where the coefficients $K_{n m}$ are given in table 3 , and

$$
\begin{aligned}
K_{\gamma_{F}}\left(Q, Q / s^{1 / j_{F}}\right)=\left[\left(\frac{\alpha_{s}(Q)}{4 \pi}\right)\left(k_{11} \ln s^{1 / j_{F}}\right)\right. & \\
& +\left(\frac{\alpha_{s}(Q)}{4 \pi}\right)^{2}\left(k_{22} \ln ^{2} s^{1 / j_{F}}+k_{21} \ln s^{1 / j_{F}}\right) \\
& \left.+\left(\frac{\alpha_{s}(Q)}{4 \pi}\right)^{3}\left(k_{33} \ln ^{3} s^{1 / j_{F}}+k_{32} \ln ^{2} s^{1 / j_{F}}+k_{31} \ln s^{1 / j_{F}}\right)+\cdots\right],
\end{aligned}
$$

where the coefficients $k_{n m}$ are given in table 4 . 
Similarly the expansion of $\eta_{\Gamma}$ is,

$$
\begin{aligned}
\eta_{\Gamma}\left(Q, Q / s^{1 / j_{F}}\right)=[ & \left(\frac{\alpha_{s}(Q)}{4 \pi}\right)\left(\eta_{11} \ln s^{1 / j_{F}}\right) \\
& +\left(\frac{\alpha_{s}(Q)}{4 \pi}\right)^{2}\left(\eta_{22} \ln ^{2} s^{1 / j_{F}}+\eta_{21} \ln s^{1 / j_{F}}\right) \\
& \left.+\left(\frac{\alpha_{s}(Q)}{4 \pi}\right)^{3}\left(\eta_{33} \ln ^{3} s^{1 / j_{F}}+\eta_{32} \ln ^{2} s^{1 / j_{F}}+\eta_{31} \ln s^{1 / j_{F}}\right)+\cdots\right]
\end{aligned}
$$

with the coefficients $\eta_{n m}$ also given in table 4. Although it does not appear in eq. (4.8), dependence on $\eta_{\Gamma}\left(\mu, \mu_{F}\right)$ will reappear if scale choices other than eq. (4.7) are made in eq. (4.2). In addition, although eq. (4.13) seems to suggest that $\eta_{\Gamma}$ begins at NLL order rather than LL, we will find that transforming between the Laplace-space $\widetilde{\sigma}(\nu)$ and the momentum-space $\sigma(\tau)$ or $R(\tau)$ to a consistent order of accuracy requires keeping anomalous dimensions in $\Omega$ to the same order as in $K$.

In eqs. (4.11) and (4.13) we have used the three-loop running of the coupling $\alpha_{s}(\mu)$ to perform the fixed-order expansion [76]. The coupling obeys the equation,

$$
\frac{d \alpha_{s}(\mu)}{d \ln \mu}=\beta\left[\alpha_{s}(\mu)\right]
$$

where the beta function has the perturbative expansion,

$$
\beta\left[\alpha_{s}\right]=-2 \alpha_{s} \sum_{n=0}^{\infty}\left(\frac{\alpha_{s}}{4 \pi}\right)^{n+1} \beta_{n} .
$$

The first few coefficients $\beta_{n}$ are given in eq. (C.1). The solution of eq. (4.14) with the 3 -loop beta function gives the 3 -loop running coupling,

$$
\begin{aligned}
\alpha_{s}(\mu)=\alpha_{s}(Q)\left\{X+\alpha_{s}(Q)\right. & \frac{\beta_{1}}{4 \pi \beta_{0}} \ln X \\
& \left.+\frac{\alpha_{s}^{2}(Q)}{16 \pi^{2}}\left[\frac{\beta_{2}}{\beta_{0}}\left(1-\frac{1}{X}\right)+\frac{\beta_{1}^{2}}{\beta_{0}^{2}}\left(\frac{\ln X}{X}+\frac{1}{X}-1\right)\right]\right\}^{-1},
\end{aligned}
$$

where

$$
X \equiv 1+\frac{\alpha_{s}(Q)}{2 \pi} \beta_{0} \ln \frac{\mu}{Q} .
$$

The pattern in eq. (4.11) and table 3 makes evident that achieving $\mathrm{N}^{k} \mathrm{LL}$ accuracy in the exponent $K$ of $\widetilde{\sigma}(\nu)$ requires the cusp anomalous dimension $\Gamma_{\text {cusp }}^{q}\left[\alpha_{s}\right]$ to order $\alpha_{s}^{k+1}$, the non-cusp part $\gamma_{F}\left[\alpha_{s}\right]$ to order $\alpha_{s}^{k}$, and the $(k+1)$-loop running of $\alpha_{s}$. Counting terms in the prefactors $H, \widetilde{F}$ consistently with the convention in table $1, \mathrm{~N}^{k} \mathrm{LL}$ accuracy can be achieved by calculating the anomalous dimensions, running coupling, and fixed-order functions $H, \widetilde{F}$ to the orders given in table 5. At each additional order in log accuracy, each column is incremented by one power of $\alpha_{s}$.

Equations (4.11) and (4.13) for $K_{F}, \omega_{F}$ can be written in closed forms that resum all the higher order terms in $\alpha_{s}$. This can be done, for example, by changing variables of 


\begin{tabular}{|c|c|c|c|c|}
\hline accuracy & $\Gamma_{F}$ & $\gamma_{F}$ & $\beta$ & $H, \widetilde{J}, \widetilde{S}$ \\
\hline $\mathrm{LL}$ & $\alpha_{s}$ & 1 & $\alpha_{s}$ & 1 \\
\hline $\mathrm{NLL}$ & $\alpha_{s}^{2}$ & $\alpha_{s}$ & $\alpha_{s}^{2}$ & 1 \\
\hline $\mathrm{NNLL}$ & $\alpha_{s}^{3}$ & $\alpha_{s}^{2}$ & $\alpha_{s}^{3}$ & $\alpha_{s}$ \\
\hline $\mathrm{N}^{3} \mathrm{LL}$ & $\alpha_{s}^{4}$ & $\alpha_{s}^{3}$ & $\alpha_{s}^{4}$ & $\alpha_{s}^{2}$ \\
\hline
\end{tabular}

\begin{tabular}{|c|c|c|c|c|}
\hline accuracy & $\Gamma_{F}$ & $\gamma_{F}$ & $\beta$ & $H, \widetilde{J}, \widetilde{S}$ \\
\hline $\mathrm{LL}$ & $\alpha_{s}$ & 1 & $\alpha_{s}$ & 1 \\
\hline $\mathrm{NLL}^{\prime}$ & $\alpha_{s}^{2}$ & $\alpha_{s}$ & $\alpha_{s}^{2}$ & $\alpha_{s}$ \\
\hline $\mathrm{NNLL}^{\prime}$ & $\alpha_{s}^{3}$ & $\alpha_{s}^{2}$ & $\alpha_{s}^{3}$ & $\alpha_{s}^{2}$ \\
\hline $\mathrm{N}^{3} \mathrm{LL}^{\prime}$ & $\alpha_{s}^{4}$ & $\alpha_{s}^{3}$ & $\alpha_{s}^{4}$ & $\alpha_{s}^{3}$ \\
\hline
\end{tabular}

Table 5. Order of anomalous dimensions, beta function, and fixed-order hard, jet, and soft functions required to achieve $\mathrm{N}^{k} \mathrm{LL}$ and $\mathrm{N}^{k} \mathrm{LL}^{\prime}$ accuracy in the exponent of $\tilde{\sigma}(\nu)$.

integration in eqs. (3.34) and (3.35) from $\mu$ to $\alpha_{s}$ using $d \mu / \mu=d \alpha_{s} / \beta\left[\alpha_{s}\right]$, yielding

$$
\begin{aligned}
& K_{\Gamma}\left(\mu, \mu_{F}\right)=\int_{\alpha_{s}\left(\mu_{F}\right)}^{\alpha_{s}(\mu)} \frac{d \alpha}{\beta[\alpha]} \Gamma_{\text {cusp }}^{q}[\alpha] \int_{\alpha_{s}\left(\mu_{F}\right)}^{\alpha} \frac{d \alpha^{\prime}}{\beta\left[\alpha^{\prime}\right]} \\
& K_{\gamma}\left(\mu, \mu_{F}\right)=\int_{\alpha_{s}\left(\mu_{F}\right)}^{\alpha_{s}(\mu)} \frac{d \alpha}{\beta[\alpha]} \gamma[\alpha], \quad \eta_{\Gamma}\left(\mu, \mu_{F}\right)=\int_{\alpha_{s}\left(\mu_{F}\right)}^{\alpha_{s}(\mu)} \frac{d \alpha}{\beta[\alpha]} \Gamma_{\text {cusp }}^{q}[\alpha]
\end{aligned}
$$

Using table 5 we obtain expressions for $K_{\Gamma}, K_{\gamma}^{F}, \eta_{\Gamma}$ order-by-order in logarithmic accuracy,

$$
\begin{gathered}
K=K^{\mathrm{LL}}+K^{\mathrm{NLL}}+K^{\mathrm{NNLL}}+\cdots \\
\eta=\eta^{\mathrm{LL}}+\eta^{\mathrm{NLL}}+\eta^{\mathrm{NNLL}}+\cdots
\end{gathered}
$$

Thus to $\mathrm{N}^{3} \mathrm{LL}$ order $[38,76]$ :

$$
\begin{aligned}
K_{\Gamma}^{\mathrm{LL}}\left(\mu, \mu_{F}\right)= & \frac{\Gamma_{0}}{4 \beta_{0}^{2}} \frac{4 \pi}{\alpha_{s}\left(\mu_{F}\right)}\left\{\ln r+\frac{1}{r}-1\right\} \\
K_{\Gamma}^{\mathrm{NLL}}\left(\mu, \mu_{F}\right)= & \frac{\Gamma_{0}}{4 \beta_{0}^{2}}\left\{\left(\frac{\Gamma_{1}}{\Gamma_{0}}-\frac{\beta_{1}}{\beta_{0}}\right)(r-\ln r-1)-\frac{\beta_{1}^{2}}{2 \beta_{0}} \ln ^{2} r\right\} \\
K_{\Gamma}^{\mathrm{NNLL}}\left(\mu, \mu_{F}\right)= & \frac{\Gamma_{0}}{4 \beta_{0}^{2}} \frac{\alpha_{s}\left(\mu_{F}\right)}{4 \pi}\left\{B_{2}\left(\frac{r^{2}-1}{2}-\ln r\right)+\left(\frac{\beta_{1} \Gamma_{1}}{\beta_{0} \Gamma_{0}}-\frac{\beta_{1}^{2}}{\beta_{0}^{2}}\right)(r-r \ln r-1)\right. \\
& \left.+\left(\frac{\Gamma_{2}}{\Gamma_{0}}-\frac{\beta_{1} \Gamma_{1}}{\beta_{0} \Gamma_{0}}\right) \frac{(1-r)^{2}}{2}\right\} \\
K_{\Gamma}^{\mathrm{N}^{3} \mathrm{LL}}\left(\mu, \mu_{F}\right)= & \frac{\Gamma_{0}}{4 \beta_{0}^{2}}\left(\frac{\alpha_{s}\left(\mu_{F}\right)}{4 \pi}\right)^{2}\left\{\left[\left(\frac{\Gamma_{1}}{\Gamma_{0}}-\frac{\beta_{1}}{\beta_{0}}\right) B_{2}+\frac{B_{3}}{2}\right] \frac{r^{2}-1}{2}-B_{2}\left(\frac{\Gamma_{1}}{\Gamma_{0}}-\frac{\beta_{1}}{\beta_{0}}\right)(r-1)\right. \\
& +\left(\frac{\Gamma_{3}}{\Gamma_{0}}-\frac{\Gamma_{2} \beta_{1}}{\Gamma_{0} \beta_{0}}+\frac{B_{2} \Gamma_{1}}{\Gamma_{0}}+B_{3}\right)\left(\frac{r^{3}-1}{3}-\frac{r^{2}-1}{2}\right)-\frac{B_{3}}{2} \ln r \\
& \left.+\frac{\beta_{1}}{2 \beta_{0}}\left(\frac{\Gamma_{2}}{\Gamma_{0}}-\frac{\Gamma_{1} \beta_{1}}{\Gamma_{0} \beta_{0}}+B_{2}\right)\left(r^{2} \ln r-\frac{r^{2}-1}{2}\right)\right\},
\end{aligned}
$$

where

$$
r \equiv \frac{\alpha_{s}(\mu)}{\alpha_{s}\left(\mu_{F}\right)}, \quad B_{2} \equiv \frac{\beta_{1}^{2}}{\beta_{0}^{2}}-\frac{\beta_{2}}{\beta_{0}}, \quad B_{3}=-\frac{\beta_{1}^{3}}{\beta_{0}^{3}}+\frac{2 \beta_{1} \beta_{2}}{\beta_{0}^{2}}-\frac{\beta_{3}}{\beta_{0}}
$$


and

$$
\begin{aligned}
\eta_{\Gamma}^{\mathrm{LL}}\left(\mu, \mu_{F}\right) & =-\frac{\Gamma_{0}}{2 \beta_{0}} \ln r \\
\eta_{\Gamma}^{\mathrm{NLL}}\left(\mu, \mu_{F}\right) & =-\frac{\Gamma_{0}}{2 \beta_{0}} \frac{\alpha_{s}\left(\mu_{F}\right)}{4 \pi}\left(\frac{\Gamma_{1}}{\Gamma_{0}}-\frac{\beta_{1}}{\beta_{0}}\right)(r-1) \\
\eta_{\Gamma}^{\mathrm{NNLL}}\left(\mu, \mu_{F}\right) & =-\frac{\Gamma_{0}}{2 \beta_{0}}\left(\frac{\alpha_{s}\left(\mu_{F}\right)}{4 \pi}\right)^{2}\left(B_{2}+\frac{\Gamma_{2}}{\Gamma_{0}}-\frac{\Gamma_{1} \beta_{1}}{\Gamma_{0} \beta_{0}}\right) \frac{r^{2}-1}{2} \\
\eta_{\Gamma}^{\mathrm{N}^{3} \mathrm{LL}}\left(\mu, \mu_{F}\right) & =-\frac{\Gamma_{0}}{2 \beta_{0}}\left(\frac{\alpha_{s}\left(\mu_{F}\right)}{4 \pi}\right)^{3}\left(\frac{\Gamma_{3}}{\Gamma_{0}}-\frac{\Gamma_{2} \beta_{1}}{\Gamma_{0} \beta_{0}}+\frac{\Gamma_{1}}{\Gamma_{0}} B_{2}+B_{3}\right)\left(r^{3}-1\right),
\end{aligned}
$$

and

$$
K_{\gamma}^{\mathrm{LL}}=0, \quad K_{\gamma}^{\mathrm{NLL}}=\eta_{\gamma}^{\mathrm{LL}}, \quad K_{\gamma}^{\mathrm{NNLL}}=\eta_{\gamma}^{\mathrm{NLL}}, \quad K_{\gamma}^{\mathrm{N} 3 \mathrm{LL}}=\eta_{\gamma}^{\mathrm{NNLL}}, \ldots
$$

These last relations mean that to get $K_{\gamma}$ on the left-hand side, use the specified formula for $\eta_{\Gamma}$ given in eq. (4.22) with the replacement $\Gamma_{n} \rightarrow \gamma_{n}$. The expressions eqs. (4.20) and (4.22) capture terms at all orders in $\alpha_{s}$ required up to $\mathrm{N}^{3} \mathrm{LL}$ accuracy. Re-expanding eqs. (4.20) and (4.22) in powers of $\alpha_{s}(Q)$ using the running coupling eq. (4.16) produces the fixed-order series shown in eqs. (4.11) and (4.13).

Note from eqs. (4.20d) and (4.22d) that $\mathrm{N}^{3} \mathrm{LL}$ accuracy requires knowing the 4-loop cusp anomalous dimension $\Gamma_{3}$, which is not known explicitly at the present time. It can however be estimated by Padé approximation, yielding approximate $\mathrm{N}^{3} \mathrm{LL}$ resummed accuracy as in [38, 71], assuming other ingredients such as non-cusp anomalous dimensions and matching coefficients are also known to sufficiently high order.

The way we divided the evolution kernel into $K_{F}$ and $\omega_{F}$ in eqs. (4.11) and (4.13) makes it appear that $\omega_{F}$ begins at a subleading order in logarithmic accuracy (NLL) compared to $K_{F}$ (LL). However, it is advisable to keep the cusp anomalous dimension to the same order of accuracy both in $K_{F}$ and $\omega_{F}$ at a given order $\mathrm{N}^{k} \mathrm{LL}$ of logarithmic accuracy, as we indicated in eq. (4.22) or table 4 . One reason for this is that $K_{\Gamma}$ and $\eta_{\Gamma}$ always appear together as in eq. (3.35) in the combination:

$$
\begin{gathered}
-j_{F} \kappa_{F} K_{F}\left(\mu, \mu_{0}\right)+\left(\mu_{0}^{j_{F}} e^{\gamma_{E}} x\right)^{-\kappa_{F} \eta_{\Gamma}\left(\mu, \mu_{0}\right)} \\
=-j_{F} \kappa_{F} \int_{\mu_{0}}^{\mu} \frac{d \mu^{\prime}}{\mu^{\prime}} \Gamma_{\text {cusp }}^{q}\left[\alpha_{s}\left(\mu^{\prime}\right)\right]\left(\ln \frac{\mu^{\prime}}{\mu_{0}}+\ln \left[\mu_{0}\left(e^{\gamma_{E}} x\right)^{1 / j_{F}}\right]\right) \\
=-j_{F} \kappa_{F} \int_{\mu_{0}}^{\mu} \frac{d \mu^{\prime}}{\mu^{\prime}} \Gamma_{\text {cusp }}^{q}\left[\alpha_{s}\left(\mu^{\prime}\right)\right] \ln \left[\mu^{\prime}\left(e^{\gamma_{E}} x\right)^{1 / j_{F}}\right],
\end{gathered}
$$

whose integrand reproduces the starting form of the anomalous dimension eq. (3.32). Thus keeping $\Gamma_{\text {cusp }}$ to the same order in $K_{F}$ and $\eta_{\Gamma}$ is necessary to remove the explicit dependence on the arbitrary scale $\mu_{0}$ we introduced into the integrand of $K_{\Gamma}$ in eq. (3.34) purely for convenience. In other words, if we had kept the evolution kernel in the form of the last line of eq. (4.24) we would automatically keep $\Gamma_{\text {cusp }}$ to the same order wherever it appears, and this is what we shall do. The remaining dependence on $\mu_{0}$ in the lower limit of the evolution integrals is cancelled by $\mu_{0}$-dependent terms in the fixed-order coefficients $H, \widetilde{J}, \widetilde{S}$. 




Figure 4. Commutative diagram for obtaining the resummed cumulant distribution from the Laplace transform. The resummed cumulant can be obtained by first inverse Laplace transforming $\widetilde{\sigma}$ with free scales, and then choosing the scales to be $\tau$-dependent. Or, it can be obtained by first fixing the scales in the Laplace transform to be $\nu$-dependent, and then inverse Laplace transforming. If expressions for $R, \widetilde{\sigma}$ are kept to all orders in $\alpha_{s}$, the two routes produce identical results, though care must be exercised at truncated finite orders to maintain equivalent accuracy. (Nota bene: when the running of the strong coupling $\alpha_{s}(\mu)$ is taken into account, the bottom route can lead to integration over the Landau pole, which can be avoided by appropriate prescriptions, but in that case the top route is preferable.)

Making the scale choices eq. (4.7) in the Laplace-transformed cross section $\tilde{\sigma}(\nu)$ in eq. (4.2) is appropriate for the purpose of resumming logs of $\nu$ if one wishes to express the distribution directly in terms of this variable. If one is interested instead in summing logs of $\tau_{a}$ in $\sigma\left(\tau_{a}\right)$ or $R\left(\tau_{a}\right)$, one might think taking the inverse Laplace transform of eq. (4.8) is the simplest thing to do, but this is actually fairly cumbersome due to the complex $\tau_{a}$ dependence after scale setting. In addition, in integrating down to $s=0$ one encounters the Landau pole in $\alpha_{s}(Q s)$ and $\alpha_{s}\left(Q s^{1 / j_{J}}\right)$ whose expansions in $\alpha_{s}(Q)$ produced the $\beta_{n^{-}}$ dependent terms in eq. (4.11). Instead, it is simpler to take the inverse Laplace transform of eq. (4.2) before choosing the scales $\mu_{F}$, and then after the transform to choose $\tau_{a}$-dependent scales, such as the canonical choice

$$
\mu=\mu_{H}=Q, \quad \mu_{F}^{\mathrm{nat}}=Q \tau_{a}^{1 / j_{F}}
$$

in calculating either $\sigma\left(\tau_{a}\right)$ or $R\left(\tau_{a}\right) .{ }^{4}$ Of course, the two orders of operations must be equivalent when the cross section is computed to all orders in $\alpha_{s}$, as illustrated in the commutative diagram figure 4. At a truncated order of accuracy, however, results may differ. We carry out these comparisons explicitly in the next subsections.

\footnotetext{
${ }^{4}$ Alternatively, one can Taylor expand eq. (4.2) around $\nu=1 / \tau_{a}$ before performing the inverse transform. This is the approach taken in the QCD resummed [15] cross section given by eq. (3.56), with the Taylor expansion performed in eq. (3.58).
} 


\subsection{Cumulant}

\subsubsection{Resummed cumulant in SCET formalism}

To obtain the cumulant $R\left(\tau_{a}\right)$, it is simplest to take the inverse Laplace transform of eq. (4.2) and integrate with respect to $\tau_{a}$, or

$$
R\left(\tau_{a}\right)=\frac{1}{2 \pi i} \int_{\mathcal{C}} \frac{d \nu}{\nu} e^{\nu \tau_{a}} \widetilde{\sigma}(\nu),
$$

which gives the result in eq. (3.48). Then making the scale choices in eq. (4.25) leads to the simple form:

$$
R\left(\tau_{a}\right)=H_{2}(Q) e^{K} \widetilde{J}\left(\partial_{\Omega}, \mu_{J}^{\text {nat }}\right)^{2} \widetilde{S}\left(\partial_{\Omega}, \mu_{S}^{\text {nat }}\right)\left[\frac{e^{\gamma_{E} \Omega}}{\Gamma(1-\Omega)}\right]
$$

where

$$
\begin{aligned}
K & =2 K_{J}\left(Q, Q \tau_{a}^{1 / j_{J}}\right)+K_{S}\left(Q, Q \tau_{a}\right), \\
\Omega & =2 \omega_{J}\left(Q, Q \tau_{a}^{1 / j_{J}}\right)+\omega_{S}\left(Q, Q \tau_{a}\right) .
\end{aligned}
$$

The scale choices eq. (4.25) are the typical "canonical" choices made in the SCET literature. Another reasonable set of choices is eq. (3.81), which lead to a form of the resummed cross section most similar to the typical QCD form eq. (3.61). With the choices eq. (3.81), we obtain for the cumulant eq. (3.48):

$$
R\left(\tau_{a}\right)=H_{2}(Q) e^{\bar{K}} \widetilde{J}\left(\partial_{\bar{\Omega}}, \bar{\mu}_{J}\right)^{2} \widetilde{S}\left(\partial_{\bar{\Omega}}, \bar{\mu}_{S}\right)\left[\frac{1}{\Gamma(1-\bar{\Omega})}\right]
$$

where

$$
\begin{aligned}
\bar{K} & =2 K_{J}\left(Q, Q \bar{\tau}_{a}^{1 / j_{J}}\right)+K_{S}\left(Q, Q \bar{\tau}_{a}\right), \\
\bar{\Omega} & =2 \omega_{J}\left(Q, Q \bar{\tau}_{a}^{1 / j_{J}}\right)+\omega_{S}\left(Q, Q \bar{\tau}_{a}\right),
\end{aligned}
$$

where $\bar{\tau}_{a}$ is defined in eq. (3.63). The two forms eqs. (4.27) and (4.29) are equivalent, when computed to all orders in $\alpha_{s}$. Truncated at a given order of logarithmic accuracy, they may differ in subleading terms but are equal at the given order of accuracy.

We proceed to classify the accuracy of logarithmic resummation for $R(\tau)$ given by eq. (4.29) before considering the distribution $\sigma\left(\tau_{a}\right)$. The discussion for $R$ will turn out to be more straightforward. With the scale choices eq. (3.81), we find from eq. (3.36),

$$
\bar{K}_{F}\left(Q, Q \bar{\tau}_{a}^{1 / j_{F}}\right)=-j_{F} \kappa_{F} K_{\Gamma}\left(Q, Q \bar{\tau}_{a}^{1 / j_{F}}\right)+K_{\gamma_{F}}\left(Q, Q \bar{\tau}_{a}^{1 / j_{F}}\right),
$$

where $K_{\Gamma}, K_{\gamma_{F}}$ have the same fixed-order expansions as in eqs. (4.11) and (4.12) with the replacement $s \rightarrow 1 / \bar{\tau}$. Meanwhile, $\bar{\Omega}=2 \bar{\omega}_{J}+\bar{\omega}_{S}$, where

$$
\bar{\omega}_{F}\left(Q, Q \bar{\tau}_{a}^{1 / j_{F}}\right)=-\kappa_{F} \eta_{\Gamma}\left(Q, Q \bar{\tau}_{a}^{1 / j_{F}}\right),
$$

with $\eta_{\Gamma}$ having the same fixed-order expansion as in eq. (4.13) with the replacement $s \rightarrow 1 / \bar{\tau}$. 
Each of the fixed-order jet and soft functions $\widetilde{J}, \widetilde{S}$ in eq. (4.29) produces a prefactor multiplying the exponentiated series. To calculate the prefactors, we use the relations

$$
\begin{aligned}
\overline{\mathcal{G}}(\bar{\Omega}) \equiv & \left(\frac{\mu}{Q \bar{\tau}}\right)^{\bar{\Omega}} \frac{1}{\Gamma(1-\bar{\Omega})}, \\
\partial_{\bar{\Omega}} \overline{\mathcal{G}}(\bar{\Omega})= & {\left[\ln \frac{\mu}{Q \bar{\tau}}+\psi(1-\bar{\Omega})\right] \mathcal{G}(\bar{\Omega}), } \\
\partial_{\bar{\Omega}}^{2} \overline{\mathcal{G}}(\bar{\Omega})= & {\left[\left(\ln \frac{\mu}{Q \bar{\tau}}+\psi(1-\bar{\Omega})\right)^{2}-\psi^{(1)}(1-\bar{\Omega})\right] \overline{\mathcal{G}}(\bar{\Omega}), } \\
\partial_{\bar{\Omega}}^{3} \overline{\mathcal{G}}(\bar{\Omega})= & {\left[\left(\ln \frac{\mu}{Q \bar{\tau}}+\psi(1-\bar{\Omega})\right)^{3}-3\left(\ln \frac{\mu}{Q \bar{\tau}}+\psi(1-\bar{\Omega})\right) \psi^{(1)}(1-\bar{\Omega})\right.} \\
& \left.+\psi^{(2)}(1-\bar{\Omega})\right] \overline{\mathcal{G}}(\bar{\Omega}), \\
\partial_{\bar{\Omega}}^{4} \overline{\mathcal{G}}(\bar{\Omega})= & {\left[\left(\ln \frac{\mu}{Q \bar{\tau}}+\psi(1-\bar{\Omega})\right)^{4}-6\left(\ln \frac{\mu}{Q \bar{\tau}}+\psi(1-\bar{\Omega})\right)^{2} \psi^{(1)}(1-\bar{\Omega})+3 \psi^{(1)}(1-\bar{\Omega})^{2}\right.} \\
& \left.+4\left(\ln \frac{\mu}{Q \bar{\tau}}+\psi(1-\bar{\Omega})\right) \psi^{(2)}(1-\bar{\Omega})-\psi^{(3)}(1-\bar{\Omega})\right] \overline{\mathcal{G}}(\bar{\Omega})
\end{aligned}
$$

and so on. Here $\psi$ is the digamma function, $\psi(z)=\Gamma^{\prime}(z) / \Gamma(z)$, and $\psi^{(n)}$ is its $n$th derivative. Note that when applied to eq. (4.29), the $\mu$ in eq. (4.33) is set equal to $Q \bar{\tau}$, so the explicit logs of $\mu / Q \bar{\tau}$ disappear.

To NLL accuracy, the equation eq. (4.27) or eq. (4.29) for $R\left(\tau_{a}\right)$ fits into the form eq. (2.10) given by CTTW, but only to NLL. At this order, the quantities in eq. (2.10) are given by

$$
\ln \Sigma=\bar{K}-\ln \Gamma(1-\bar{\Omega}), \quad C\left(\alpha_{s}\right)=1,
$$

but beyond NLL we see that parts of the CTTW "exponent" are actually generated by the parts of the fixed-order functions $\widetilde{F}$ that contain derivative operators $\partial_{\bar{\Omega}}$ [30]. The forms eqs. (3.61) and (3.82) are properly generalized forms of eq. (2.10), where some terms in the $\tau_{a}$-dependent function $\Sigma\left(\tau_{a}, \alpha_{s}\right)$ are produced by the differential operator $\widehat{T}\left(\bar{E}^{\prime}\right)$, or equivalently in the forms eqs. (4.27) and (4.29) by the differential operators $\widetilde{F}\left(\partial_{\Omega, \bar{\Omega}}\right)$.

\subsubsection{Computing Laplace transform and cumulant to consistent accuracy}

Note: in this subsection, we will ignore the running of $\alpha_{s}$, treating it as a constant. As a reminder, we will use the shorthand $a_{s}=\alpha_{s} / 4 \pi$.

Above we have defined $\mathrm{N}^{k} \mathrm{LL}$ or $\mathrm{N}^{k} \mathrm{LL}^{\prime}$ accuracy by the number of terms in the exponent of $\widetilde{\sigma}(\nu)$ in eq. (2.6) or eq. (4.8) that are accurately predicted. In computing the $\tau_{a}$-distribution in momentum space, the counting of logarithmic accuracy can be a little trickier. For instance, expressions like eq. (3.82), eq. (4.27), eq. (4.29) for the cumulant $R\left(\tau_{a}\right)$ contain a series of derivative operators acting on a gamma function containing large logs. How are the logs generated by these derivatives to be counted when computing to $\mathrm{N}^{k} \mathrm{LL}$ or $\mathrm{N}^{k} \mathrm{LL}^{\prime}$ accuracy? 
Now, the resummed cumulant $R\left(\tau_{a}\right)$ given by eq. (4.27) or eq. (4.29) is supposed to be equal to the inverse transform eq. (4.26) of the resummed Laplace-transformed cross section $\widetilde{\sigma}(\nu)$ in eq. (4.8). This is exactly true when both formulae are computed to all orders in $\alpha_{s}$. In practice, of course, we have to truncate the accuracy of each at some finite order, so the exact equivalence between the two forms cannot in practice be maintained. Here we will explore this relationship by comparing the results of truncating these formulae for $\widetilde{\sigma}(\nu)$ or $R(\tau)$ at a finite logarithmic accuracy. We then prescribe how to compute $R\left(\tau_{a}\right)$ so that the equivalence between it and $\widetilde{\sigma}(\nu)$ in eq. (4.8) computed at a given order of resummed accuracy is maintained as best as possible. We will give a prescription for truncating ingredients of the resummed $R\left(\tau_{a}\right)$ so that it is at least as accurate as $\widetilde{\sigma}(\nu)$ at $\mathrm{N}^{k} \mathrm{LL}$ or $\mathrm{N}^{k} \mathrm{LL}^{\prime}$ accuracy, although subleading terms will differ.

First, note that we defined $\mathrm{N}^{k} \mathrm{LL}$ accuracy for $\widetilde{\sigma}(\nu)$ by the number of terms in the exponent $K$ that are included, given by table 3 . $\mathrm{N}^{k} \mathrm{LL}$ accuracy for $\widetilde{\sigma}(\nu)$ is achieved by calculating anomalous dimensions and the fixed-order coefficients to the orders given in table 5. Inverse Laplace transforming the $\mathrm{N}^{k} \mathrm{LL} \widetilde{\sigma}(\nu)$ by eq. (4.26) must then produce the expression eq. (4.29) with $\bar{K}, \bar{\Omega}$ and the fixed-order coefficients $H, \widetilde{J}, \widetilde{S}$ computed to the same orders as given by table 5 . In particular, this produces an expression for $\bar{\Omega}$ computed with the cusp anomalous dimension kept to the same order as in $\bar{K}$ at $\mathrm{N}^{k} \mathrm{LL}$ accuracy, i.e. to order $\alpha_{s}^{k+1}$, as specified in table 5. This is the case even though, by itself, $\omega_{F}=-\kappa_{F} \eta_{\Gamma}$ given by eq. (4.13) appears to begin at one lower logarithmic order than $K_{\Gamma}$ in eq. (4.11). Even though it is not strictly required for achieving $\mathrm{N}^{k} \mathrm{LL}$ accuracy, keeping $\Gamma_{\text {cusp }}$ to the same order in $\bar{\Omega}$ as in $\bar{K}$ is necessary for keeping the expressions eq. (4.8) for $\tilde{\sigma}(\nu)$ and eq. (4.29) for $R\left(\tau_{a}\right)$ exactly equal to each other upon Laplace transformation. [See also comments after eq. (4.24) on benefits of computing $\bar{\Omega}$ to this order.]

This is not by itself sufficient, however, for we need to consider the contribution of the derivative operator terms in eq. (4.29) as well. To illustrate in a simple example the effect of these terms (being somewhat schematic), consider a distribution $\widetilde{\sigma}(\nu, \mu)$ in Laplace space which is made up of a product of transformed functions $\widetilde{F}$, each of which satisfies an RG equation of the form

$$
\mu \frac{d}{d \mu} \widetilde{F}(\nu, \mu)=2 \Gamma_{F}\left[a_{s}\right] \ln \left(\frac{\mu \nu e^{\gamma_{E}}}{Q}\right)+\gamma_{F}\left[a_{s}\right],
$$

where $\Gamma_{F}, \gamma_{F}$ have the expansions in $a_{s}$,

$$
\Gamma_{F}\left[a_{s}\right]=\sum_{n=0}^{\infty} \Gamma_{F, n} a_{s}^{n+1}, \quad \gamma_{F}\left[a_{s}\right]=\sum_{n=0}^{\infty} \gamma_{F, n} a_{s}^{n+1} .
$$

Then, the resummed $\widetilde{\sigma}(\nu, \mu)$ is a product of functions of the form

$$
\widetilde{F}(\nu, \mu)=\widetilde{F}\left(\nu, \mu_{F}\right) e^{K_{F}\left(\mu, \mu_{F}\right)}\left(\frac{\mu_{F} \nu e^{\gamma_{E}}}{Q}\right)^{\omega_{F}\left(\mu, \mu_{F}\right)},
$$

where

$$
K_{F}\left(\mu, \mu_{F}\right)=\Gamma_{F} \ln ^{2} \frac{\mu}{\mu_{F}}+\gamma_{F} \ln \frac{\mu}{\mu_{F}}, \quad \omega_{F}\left(\mu, \mu_{F}\right)=2 \Gamma_{F} \ln \frac{\mu}{\mu_{F}}
$$


For simplicity, we will write the full cross section $\widetilde{\sigma}$ in a schematic form, in terms of only one of these evolution factors,

$$
\widetilde{\sigma}(\nu, \mu)=\widetilde{\sigma}\left(\nu, \mu_{0}\right) e^{K\left(\mu, \mu_{0}\right)}\left(\frac{\mu_{0} \nu e^{\gamma_{E}}}{Q}\right)^{\Omega\left(\mu, \mu_{0}\right)},
$$

although the number of evolution factors does not affect the conclusions drawn from this discussion. One can think of $\mu_{0}$ as representing a set of scales $\mu_{i}$ for each evolution factor. Upon choosing the scales $\mu_{0}=Q\left(\nu e^{\gamma_{E}}\right)^{-1}$ and $\mu=Q$ yields the expression

$$
\widetilde{\sigma}(\nu)=C\left(a_{s}\right) e^{\Gamma \tilde{L}^{2}+\gamma \tilde{L}}
$$

where $\tilde{L} \equiv \ln \left(\nu e^{\gamma_{E}}\right)$, and the coefficient $C$ contains no logs,

$$
C\left(a_{s}\right) \equiv \widetilde{\sigma}\left(\nu, Q\left(\nu e^{\gamma_{E}}\right)^{-1}\right)=1+\sum_{n=1}^{\infty} C_{n} a_{s}^{n}
$$

Taking the inverse Laplace transform of $\widetilde{\sigma}(\nu, \mu)$ in eq. (4.39) before fixing the scale $\mu_{0}$, we obtain for the cumulant distribution in momentum space,

$$
R(\tau, \mu)=\int_{\mathcal{C}} \frac{d \nu}{2 \pi i} \frac{e^{\nu \tau}}{\nu} \widetilde{\sigma}(\nu)=C\left(a_{s}\right) e^{K\left(\mu, \mu_{0}\right)} e^{\Gamma \partial_{\Omega}^{2}+\gamma \partial_{\Omega}}\left(\frac{\mu_{0}}{Q \tau}\right)^{\Omega\left(\mu, \mu_{0}\right)} \frac{e^{\gamma_{E} \Omega\left(\mu, \mu_{0}\right)}}{\Gamma\left(1-\Omega\left(\mu, \mu_{0}\right)\right)}
$$

The differential operator $e^{\Gamma \partial_{\Omega}^{2}+\gamma \partial_{\Omega}}$ here is the equivalent of the product of operators $\widetilde{J}\left(\partial_{\Omega}\right)^{2} \widetilde{S}\left(\partial_{\Omega}\right)$ in the full SCET expression eq. (3.48) or the differential operator terms in eq. (3.82). Thus the order to which we truncated this operator corresponds to the order to which we truncate the jet and soft functions in SCET or the differential operators in the dQCD resummed cumulant.

In eq. (4.42) we can choose the scale $\mu_{0}$ to be $Q \tau$ or $Q \bar{\tau}=Q \tau e^{-\gamma_{E}}$. Here, we will pick the canonical choice $\mu_{0}=Q \tau$, and the same scale $\mu=Q$ as in eq. (4.40). Then we obtain

$$
R(\tau)=C e^{\Gamma L^{2}+\gamma L} e^{\Gamma \partial_{\Omega}^{2}} \frac{e^{\gamma_{E}(\Omega+\gamma)}}{\Gamma(1-\Omega-\gamma)}
$$

where

$$
L \equiv \ln \frac{1}{\tau}, \quad \Omega=2 \Gamma L
$$

and where we have used the translation operator $e^{\gamma \partial_{\Omega}}$ in eq. (4.42) to shift the arguments in last factor in eq. (4.43) from $\Omega$ to $\Omega+\gamma$.

Since the factor $C\left(a_{s}\right)$ is common to both $\widetilde{\sigma}(\nu)$ in eq. (4.40) and $R(\tau)$ in eq. (4.43), we will not explicitly include it in comparing the relative accuracy of the two expressions. We focus on comparing how accurately the remaining explicitly log-dependent factors in $R(\tau)$ in eq. (4.43) reproduce the exponentiated logs in $\widetilde{\sigma}(\nu)$ in eq. (4.40) upon Laplace transformation. It is these terms that determine the shape of the spectrum in $\tau$.

The two formulae eq. (4.39) for $\widetilde{\sigma}(\nu, \mu)$ and eq. (4.42) are related by Laplace transformation before fixing the scale $\mu_{0}$ to be $\nu$-dependent or $\tau$-dependent. This relation is represented by the top arrow in figure 4. Fixing the scales $\mu=Q$ and $\mu_{0}=Q\left(\nu e^{\gamma_{E}}\right)^{-1}$ 
or $\mu_{0}=Q \tau$ is represented by the vertical arrows. It should follow that transforming between $\widetilde{\sigma}(\nu)$ and $R(\tau)$ on the bottom of figure 4 after fixing the scales should also be valid. Evaluating the transform in practice is more involved, however, due to the additional $\nu$ or $\tau$ dependence in the scales (at least once the running of $\alpha_{s}$ is restored), but we can compute at some truncated order in $\alpha_{s}$ to compare the accuracy to which the transform on the bottom of figure 4 is achieved.

Now, transforming $R(\tau)$ in eq. (4.43) back to $\tilde{\sigma}(\nu)$ using

$$
\frac{1}{\nu} \widetilde{\sigma}(\nu)=\int_{0}^{\infty} d \tau e^{-\nu \tau} R(\tau)
$$

will yield exactly the same result as eq. (4.40), if all objects in both expressions are computed to all orders in $\alpha_{s}$. However, when truncated at a given logarithmic accuracy, the inability to evaluate the infinite series of differential operators in $e^{\Gamma \partial_{\Omega}^{2}}$ in eq. (4.43) prevents this from being exactly realized. An explicit example will serve to illustrate this.

First, consider $\widetilde{\sigma}(\nu)$ given, after fixing scales, by eq. (4.40), to $\mathrm{N}^{3} \mathrm{LL}$ accuracy [equation appears in color]:

$$
\begin{aligned}
\tilde{\sigma}(\nu)=C\left(a_{s}\right) \exp \left\{a_{s}\left(\Gamma_{0} \tilde{L}^{2}+\gamma_{0} \tilde{L}\right)\right. & \\
& +a_{s}^{2}\left(\Gamma_{1} \tilde{L}^{2}+\gamma_{1} \tilde{L}\right) \\
& \left.\quad+a_{s}^{3}\left(\Gamma_{2} \tilde{L}^{2}+\gamma_{2} \tilde{L}\right)+a_{s}^{4} \Gamma_{3} \tilde{L}^{2}+\cdots\right\},
\end{aligned}
$$

where we have colored the terms at LL accuracy in red, NLL in green, NNLL in blue, and $\mathrm{N}^{3} \mathrm{LL}$ in purple. Now expanding eq. (4.46) in fixed orders in $a_{s}$ up to $\mathcal{O}\left(a_{s}^{3}\right)$ [color]:

$$
\begin{aligned}
\tilde{\sigma}(\nu)=C\left(a_{s}\right) & \left\{1+a_{s}\left(\Gamma_{0} \tilde{L}^{2}+\gamma_{0} \tilde{L}\right)\right. \\
& +a_{s}^{2}\left[\frac{\Gamma_{0}^{2}}{2} \tilde{L}^{4}+\Gamma_{0} \gamma_{0} \tilde{L}^{3}+\left(\frac{\gamma_{0}^{2}}{2}+\Gamma_{1}\right) \tilde{L}^{2}+\gamma_{1} \tilde{L}\right] \\
& +a_{s}^{3}\left[\frac{\Gamma_{0}^{3}}{6} \tilde{L}^{6}+\frac{\Gamma_{0}^{2} \gamma_{0}}{2} \tilde{L}^{5}+\left(\frac{\Gamma_{0} \gamma_{0}^{2}}{2}+\Gamma_{1} \Gamma_{0}\right) \tilde{L}^{4}+\left(\frac{\gamma_{0}^{3}}{6}+\Gamma_{1} \gamma_{0}+\Gamma_{0} \gamma_{1}\right) \tilde{L}^{3}\right. \\
& \left.\left.+\left(\gamma_{1} \gamma_{0}+\Gamma_{2}\right) \tilde{L}^{2}+\gamma_{2} \tilde{L}\right]\right\},
\end{aligned}
$$

where the colors indicate which terms in the fixed-order expansion are generated by expanding the exponent in eq. (4.46) to the corresponding order in logarithmic accuracy. At $\mathrm{N}^{k} \mathrm{LL}$ order, the first log that is missing in the exponent of eq. (4.46) is $\sim a_{s}^{k+1} \gamma_{k} \tilde{L}$. Thus, in eq. (4.47), the largest log that is missing at $\mathcal{O}\left(\alpha_{s}^{k+n}\right)$ in the fixed-order expansion of the exponentiated $\operatorname{logs} e^{\Gamma \tilde{L}^{2}+\gamma \tilde{L}}$ in $\widetilde{\sigma}(\nu)$ is:

largest missing $\log$ in $\mathrm{N}^{k} \mathrm{LL} \widetilde{\sigma}(\nu)$ at fixed-order $\mathcal{O}\left(a_{s}^{k+n}\right)$ :

$$
\left(a_{s}^{k+1} \gamma_{k} \tilde{L}\right)\left(a_{s}^{n-1} \frac{\Gamma_{0}^{n-1}}{(n-1) !} \tilde{L}^{2 n-2}\right)=a_{s}^{k+n} \frac{\Gamma_{0}^{n-1} \gamma_{k}}{(n-1) !} \tilde{L}^{2 n-1}
$$


We will use eqs. (4.47) and (4.48) to compare which terms are accurately predicted by the Laplace transform of the cumulant $R(\tau)$ truncated at a given order. It is reasonable to expect that a definition or prescription for $\mathrm{N}^{k} \mathrm{LL}$ accuracy in $R(\tau)$ should not mis-predict any logs that are larger than eq. (4.48) upon Laplace transformation.

Now consider the expansion of the cumulant $R(\tau)$ in eq. (4.43) with the anomalous dimensions kept to the same accuracy. The factor

$$
e^{K(Q, Q \tau)}=e^{\Gamma L^{2}+\gamma L}
$$

will take exactly the same form as the expansion in eq. (4.47) for $\tilde{\sigma}(\nu)$, with each $\tilde{L} \equiv$ $\ln \left(\nu e^{\gamma_{E}}\right)$ replaced by $L \equiv \ln (1 / \tau)$. However, the powers $\tilde{L}^{n}$ and $L^{n}$ are not exact transforms of each other, as we see from eqs. (B.3) and (B.5), each contains a series of lower-order $\operatorname{logs} L^{n-k}$ or $\tilde{L}^{n-k}$ for $0 \leq k \leq n$. The extra terms in the transform of each power $\tilde{L}^{n}$ are provided by the expansion of the extra factors in eq. (4.43),

$$
\begin{aligned}
e^{\Gamma \partial_{\Omega}^{2}} \frac{e^{\gamma_{E}(\Omega+\gamma)}}{\Gamma(1-\Omega-\gamma)}=\left(1+\Gamma \partial_{\Omega}^{2}+\frac{\Gamma^{2}}{2} \partial_{\Omega}^{4}+\frac{\Gamma^{3}}{6} \partial_{\Omega}^{6}+\cdots\right) & \\
\times & {\left[1-\frac{\pi^{2}}{12}(\Omega+\gamma)^{2}-\frac{\zeta_{3}}{3}(\Omega+\gamma)^{3}+\frac{\pi^{4}}{1440}(\Omega+\gamma)^{4}\right.} \\
& \left.+\frac{5 \pi^{2} \zeta_{3}-36 \zeta_{5}}{180}(\Omega+\gamma)^{5}+\left(\frac{\zeta_{3}^{2}}{18}-\frac{\pi^{6}}{24192}\right)(\Omega+\gamma)^{6}+\cdots\right],
\end{aligned}
$$

or, evaluating the derivatives up to terms contributing at $\mathcal{O}\left(a_{s}^{3}\right)$,

$$
\begin{aligned}
e^{\Gamma \partial_{\Omega}^{2}} \frac{e^{\gamma_{E}(\Omega+\gamma)}}{\Gamma(1-\Omega-\gamma)}= & 1-\frac{\pi^{2}}{12}(\Omega+\gamma)^{2}-\frac{\zeta_{3}}{3}(\Omega+\gamma)^{3} \\
& +\Gamma\left[-\frac{\pi^{2}}{6}-2 \zeta_{3}(\Omega+\gamma)+\frac{\pi^{4}}{120}(\Omega+\gamma)^{2}\right] \\
& +\frac{\Gamma^{2}}{2}\left[\frac{\pi^{4}}{60}+\left(\frac{10 \pi^{2}}{3} \zeta_{3}-24 \zeta_{5}\right)(\Omega+\gamma)\right] \\
& +\frac{\Gamma^{3}}{6}\left(40 \zeta_{3}^{2}-\frac{5 \pi^{6}}{168}\right)+\cdots,
\end{aligned}
$$

recalling $\Omega=2 \Gamma L$ for fixed $a_{s}$. The derivatives can also be computed from the series expansions of the expressions in eq. (4.33).

Now to compute $R\left(\tau_{a}\right)$ up to $\mathcal{O}\left(a_{s}^{3}\right)$, we multiply together eqs. (4.49) and (4.51), and find for the fixed-order expansion of $R\left(\tau_{a}\right)$ [color],

$$
\begin{aligned}
R(\tau)=\mathscr{L}^{-1}( & \frac{C\left(a_{s}\right)}{\nu}\left\{1+a_{s}\left(\Gamma_{0} \tilde{L}^{2}+\gamma_{0} \tilde{L}\right)\right. \\
+ & a_{s}^{2}\left[\frac{\Gamma_{0}^{2}}{2} \tilde{L}^{4}+\Gamma_{0} \gamma_{0} \tilde{L}^{3}+\left(\frac{\gamma_{0}^{2}}{2}+\Gamma_{1}\right) \tilde{L}^{2}+\gamma_{1} \tilde{L}\right] \\
& +a_{s}^{3}\left[\frac{\Gamma_{0}^{3}}{6} \tilde{L}^{6}+\frac{\Gamma_{0}^{2} \gamma_{0}}{2} \tilde{L}^{5}+\left(\frac{\Gamma_{0} \gamma_{0}^{2}}{2}+\Gamma_{1} \Gamma_{0}\right) \tilde{L}^{4}\right. \\
& \left.\left.\left.+\left(\frac{\gamma_{0}^{3}}{6}+\Gamma_{1} \gamma_{0}+\Gamma_{0} \gamma_{1}\right) \tilde{L}^{3}+\left(\gamma_{1} \gamma_{0}+\Gamma_{2}\right) \tilde{L}^{2}+\gamma_{2} \tilde{L}\right]\right\}\right) .
\end{aligned}
$$


where $\mathscr{L}^{-1}\left(\tilde{L}^{n} / \nu\right)$ is given explicitly by eq. (B.5). That is, the Laplace transform of $R(\tau)$ is exactly the expansion eq. (4.47) of $\widetilde{\sigma}(\nu)$ up to the terms at $\mathcal{O}\left(a_{s}^{3}\right)$ that are predicted at $\mathrm{N}^{3} \mathrm{LL}$ accuracy. However, to achieve this result it was necessary to keep the expansion of the derivative operator $e^{\Gamma \partial_{\Omega}^{2}}$ in eq. (4.51) up to the terms of $\mathcal{O}\left(a_{s}^{3}\right)$. This operator corresponds to terms in the $\widetilde{F}\left(\partial_{\Omega}\right)$ operators in the SCET resummed cumulant eq. (4.27) or eq. (4.29), or the $\partial_{\bar{E}^{\prime}}$ terms in the QCD-inspired form eq. (3.82). This tells us that the Laplace transform of $R(\tau)$ reproduces the expansion eq. (4.47) of $\widetilde{\sigma}(\nu)$ exactly only up to the order in $a_{s}$ to which the differential operators are kept. Thus, to reproduce the $\mathrm{N}^{k} \mathrm{LL} \widetilde{\sigma}(\nu)$ in eq. (4.47) exactly to arbitrary order in $a_{s}$, the differential operators in the expansion of $e^{\Gamma \partial_{\Omega}^{2}}$ would have to kept to infinite order.

Luckily, we will be able to reproduce $\widetilde{\sigma}(\nu)$ to $\mathrm{N}^{k} \mathrm{LL}$ accuracy by keeping only a finite number of these derivative operators, but to higher order than table 5 might suggest. To see this, let's first start naïvely, applying the rules in table 1 to evaluate $R(\tau)$ given by eq. (4.43) at NLL order. When table 1 tells us to keep the $\widetilde{J}, \widetilde{S}$ functions to tree level at NLL, interpreted naïvely we also truncate the operator $e^{\Gamma \partial_{\Omega}^{2}}$ in eq. (4.43) to tree level. Then we would obtain for $R(\tau)$ :

$$
\begin{aligned}
R(\tau) \stackrel{\mathrm{NLL}}{=} & \exp \left[a_{s}\left(\Gamma_{0} L^{2}+\gamma_{0} L\right)+a_{s}^{2} \Gamma_{1} L^{2}\right] \frac{\exp \left\{\gamma_{E}\left[a_{s}\left(2 \Gamma_{0} L+\gamma_{0}\right)+a_{s}^{2}\left(2 \Gamma_{1} L\right)\right]\right\}}{\Gamma\left[1-a_{s}\left(2 \Gamma_{0} L+\gamma_{0}\right)-a_{s}^{2}\left(2 \Gamma_{1} L\right)\right]} \\
= & 1+a_{s}\left(\Gamma_{0} L^{2}+\gamma_{0} L\right) \\
& +a_{s}^{2}\left[\frac{\Gamma_{0}^{2}}{2}\left(L^{4}-\frac{2 \pi^{2}}{3} L^{2}\right)+\Gamma_{0} \gamma_{0}\left(L^{3}-\frac{\pi^{2}}{3} L\right)+\frac{\gamma_{0}^{2}}{2}\left(L^{2}-\frac{\pi^{2}}{6}\right)+\Gamma_{1} L^{2}\right]+\cdots,
\end{aligned}
$$

expanded to $\mathcal{O}\left(a_{s}^{2}\right)$. Comparing to eq. (B.5), the first thing we notice is that eq. (4.53) is missing the $a_{s}\left(\pi^{2} / 6\right)$ term required to accompany $L^{2}$ for it to Laplace transform properly to $\tilde{L}^{2}$. This is fine at NLL accuracy, since the missing $\mathcal{O}\left(\alpha_{s}\right)$ term corresponds to a subleading term in the $\widetilde{\sigma}(\nu)$ counting [see eq. (4.47)]. However, we then notice eq. (4.53) also has the wrong $a_{s}^{2} \pi^{2} L^{2}$ term required to make $L^{4}$ transform properly to $\tilde{L}^{4}$. This would lead to an incorrect $a_{s}^{2} \tilde{L}^{2}$ term in the Laplace transform of eq. (4.53), which should equal eq. (4.47). [Continuing to $\mathcal{O}\left(a_{s}^{3}\right)$ we would find the $a_{s}^{3} L^{4}$ term in the Laplace transform of $R(\tau)$ also incorrect.] The mismatch occurs in terms that are completely green (NLL) in eq. (4.47), thus making the supposedly NLL $R(\tau)$ in eq. (4.53) objectively less accurate than $\widetilde{\sigma}(\nu)$ in eq. (4.47) at NLL. The similar problem occurs at $\mathrm{N}^{k} \mathrm{LL}$ order if table 5 is used to keep the differential operator $e^{\Gamma \partial_{\Omega}^{2}}$ to the same order as the fixed-order coefficients $C\left(a_{s}\right)$. To be precise, the largest $\log$ at a given fixed order in $a_{s}$ that is incorrectly predicted at $\mathrm{N}^{k} \mathrm{LL}$ by using eq. (4.43) with the differential operator kept to the order specified for the fixed-order coefficients in table 5 is:

$$
\begin{aligned}
& \text { largest incorrect } \log \text { in } \mathrm{N}^{k} \mathrm{LL} R(\tau) \text { at fixed-order } \mathcal{O}\left(\alpha_{s}^{k+n}\right) \text { : } \\
& \sim\left[a_{s}^{k} \Gamma_{0}^{k}(\#)\right]\left(a_{s}^{n} \frac{\Gamma_{0}^{n}}{n !} L^{2 n}\right)=a_{s}^{k+n}(\#) \frac{\Gamma_{0}^{n+k}}{n !} L^{2 n}
\end{aligned}
$$

where (\#) is the constant generated by taking the derivative $\partial_{\Omega}^{2 k}$ of the $(\Omega+\gamma)^{2 k}$ term in the series expansion eq. (4.50). We see the term in eq. (4.54) is larger (by one power 
of a $\log$ ) than the largest $\log$ missing in the $\mathrm{N}^{k} \mathrm{LL} \widetilde{\sigma}(\nu)$ identified in eq. (4.48). This is undesirable for an expression labeled to be $\mathrm{N}^{k} \mathrm{LL}$ accurate, which should contain the equivalent information as in the $\mathrm{N}^{k} \mathrm{LL}$ Laplace transform.

These deficiencies are remedied by applying the $\mathrm{N}^{k} \mathrm{LL}^{\prime}$ counting in table 5 to the differential operator $e^{\Gamma \partial_{\Omega}^{2}}$ and thus expanding it to one higher order than at $\mathrm{N}^{k} \mathrm{LL}$ order. For example, at NLL' order, with the differential operator $e^{\Gamma \partial_{\Omega}^{2}}$ expanded to first order, $1+\Gamma \partial_{\Omega}^{2}$, and truncating this to $\mathcal{O}\left(\alpha_{s}\right), 1+a_{s} \Gamma_{0} \partial_{\Omega}^{2}$, we find that $R(\tau)$ has the expansion to $\mathcal{O}\left(\alpha_{s}^{3}\right)$ :

$$
\begin{gathered}
R(\tau) \stackrel{\mathrm{NLL}^{\prime}}{=} C\left(a_{s}\right)\left\{1+a_{s}\left[\Gamma_{0}\left(L^{2}-\frac{\pi^{2}}{6}\right)+\gamma_{0} L\right]\right. \\
+a_{s}^{2}\left[\frac{\Gamma_{0}^{2}}{2}\left(L^{4}-\pi^{2} L^{2}-8 \zeta_{3} L\right)+\Gamma_{0} \gamma_{0}\left(L^{3}-\frac{\pi^{2}}{2} L-2 \zeta_{3}\right)+\frac{\gamma_{0}^{2}}{2}\left(L^{2}-\frac{\pi^{2}}{6}\right)+\Gamma_{1} L^{2}\right] \\
+a_{s}^{3}\left[\frac{\Gamma_{0}^{3}}{6}\left(L^{6}-\frac{5 \pi^{2}}{2} L^{4}-40 \zeta_{3} L^{3}\right)+\frac{\Gamma_{0}^{2} \gamma_{0}}{2}\left(L^{5}-\frac{5 \pi^{2}}{3} L^{3}-20 \zeta_{3} L^{2}\right)\right. \\
+\frac{\Gamma_{0} \gamma_{0}^{2}}{2}\left(L^{4}-\pi^{2} L^{2}-8 \zeta_{3} L\right)+\Gamma_{0} \Gamma_{1}\left(L^{4}-\frac{5 \pi^{2}}{6} L^{2}-4 \zeta_{3} L\right) \\
\left.\left.+\frac{\gamma_{0}^{3}}{6}\left(L^{3}-\frac{\pi^{2}}{2} L-2 \zeta_{3}\right)+\Gamma_{1} \gamma_{0}\left(L^{3}-\frac{\pi^{2}}{3} L\right)\right]\right\} .
\end{gathered}
$$

Note that more of the displayed terms are now the correct inverse Laplace transforms of $\tilde{L}^{n} / \nu$, according to eq. (B.5), in contrast to eq. (4.53) at unprimed NLL order. Not all the terms in eq. (4.55) at $\mathcal{O}\left(a_{s}^{2}\right)$ and $\mathcal{O}\left(a_{s}^{3}\right)$ satisfy this property - for example, the $a_{s}^{2} \Gamma_{1} L^{2}$ term. However, there are enough logs correctly predicted in eq. (4.55) that its Laplace transform reproduces all the terms in the fixed-order expansion eq. (4.47) of $\widetilde{\sigma}(\nu)$ that are supposed to be correct at NLL' order [the green terms in eq. (4.47)]. That is, at NLL' order,

$$
\begin{aligned}
R(\tau)=\mathscr{L}^{-1}( & \frac{1}{\nu} C\left(a_{s}\right)\left\{1+a_{s}\left[\Gamma_{0} \tilde{L}^{2}+\gamma_{0} \tilde{L}\right]+a_{s}^{2}\left[\frac{\Gamma_{0}^{2}}{2} \tilde{L}^{4}+\Gamma_{0} \gamma_{0} \tilde{L}^{3}+\left(\frac{\gamma_{0}^{2}}{2}+\Gamma_{1}\right) \tilde{L}^{2}\right]\right. \\
& \left.\left.+a_{s}^{3}\left[\frac{\Gamma_{0}^{3}}{6} \tilde{L}^{6}+\frac{\Gamma_{0}^{2} \gamma_{0}}{2} \tilde{L}^{5}+\left(\frac{\Gamma_{0} \gamma_{0}^{2}}{2}+\Gamma_{0} \Gamma_{1}\right) \tilde{L}^{4}+\left(\frac{\gamma_{0}^{3}}{6}+\Gamma_{1} \gamma_{0}\right) \tilde{L}^{3}+\cdots\right]\right\}\right),
\end{aligned}
$$

where the $\cdots$ on the last line indicate terms at $\mathcal{O}\left(a_{s}^{2}\right)$ and higher order that are truly subleading at $\mathrm{NLL}^{\prime}$ accuracy in $\widetilde{\sigma}(\nu)$. The largest log in the fixed-order expansion of $R(\tau)$ given by eq. (4.43) that is missing at $\mathrm{N}^{k} \mathrm{LL}^{\prime}$ order is:

$$
\begin{aligned}
& \text { largest incorrect } \log \text { in } \mathrm{N}^{k} \mathrm{LL}^{\prime} R(\tau) \text { at fixed-order } \mathcal{O}\left(\alpha_{s}^{k+n}\right): \\
& \sim\left[a_{s}^{k+1} \Gamma_{0}^{k+1}(\#)\right]\left(a_{s}^{n-1} \frac{\Gamma_{0}^{n-1}}{(n-1) !} L^{2 n-2}\right)=a_{s}^{k+n} \frac{\Gamma_{0}^{n+k}}{(n-1) !} L^{2 n-2},
\end{aligned}
$$

which is two powers of logs smaller than the corresponding missing log in the $\mathrm{N}^{k} \mathrm{LL} R(\tau)$ in eq. (4.54), and one power smaller than the corresponding missing log in the $\mathrm{N}^{k} \mathrm{LL}$ or $\mathrm{N}^{k} \mathrm{LL}^{\prime}$ $\widetilde{\sigma}(\nu)$ in eq. (4.48). Thus, the Laplace transform of the $\mathrm{N}^{k} L L^{\prime}$ cumulant $R(\tau)$ evaluated using eq. (4.43) does reproduce the $\mathrm{N}^{k} \mathrm{LL}^{\prime} \widetilde{\sigma}(\nu)$ evaluated from eq. (4.40). 


\begin{tabular}{|c|c|c|c|c|c|}
\hline accuracy & $\Gamma_{F}$ & $\gamma_{F}$ & $\beta$ & $\widetilde{J}, \widetilde{S}\left(\partial_{\Omega}\right.$ terms $)$ & $c_{H, J, S}$ \\
\hline $\mathrm{LL}$ & $\alpha_{s}$ & 1 & $\alpha_{s}$ & 1 & 1 \\
\hline $\mathrm{NLL}$ & $\alpha_{s}^{2}$ & $\alpha_{s}$ & $\alpha_{s}^{2}$ & $\alpha_{s}$ & 1 \\
\hline $\mathrm{NNLL}$ & $\alpha_{s}^{3}$ & $\alpha_{s}^{2}$ & $\alpha_{s}^{3}$ & $\alpha_{s}^{2}$ & $\alpha_{s}$ \\
\hline $\mathrm{N}^{3} \mathrm{LL}$ & $\alpha_{s}^{4}$ & $\alpha_{s}^{3}$ & $\alpha_{s}^{4}$ & $\alpha_{s}^{3}$ & $\alpha_{s}^{2}$ \\
\hline
\end{tabular}

\begin{tabular}{|c|c|c|c|c|}
\hline accuracy & $\Gamma_{F}$ & $\gamma_{F}$ & $\beta$ & $H, \widetilde{J}, \widetilde{S}$ (full) \\
\hline $\mathrm{LL}$ & $\alpha_{s}$ & 1 & $\alpha_{s}$ & 1 \\
\hline $\mathrm{NLL}^{\prime}$ & $\alpha_{s}^{2}$ & $\alpha_{s}$ & $\alpha_{s}^{2}$ & $\alpha_{s}$ \\
\hline $\mathrm{NNLL}^{\prime}$ & $\alpha_{s}^{3}$ & $\alpha_{s}^{2}$ & $\alpha_{s}^{3}$ & $\alpha_{s}^{2}$ \\
\hline $\mathrm{N}^{3} \mathrm{LL}^{\prime}$ & $\alpha_{s}^{4}$ & $\alpha_{s}^{3}$ & $\alpha_{s}^{4}$ & $\alpha_{s}^{3}$ \\
\hline
\end{tabular}

Table 6. Order of anomalous dimensions, beta function, and fixed-order hard, jet, and soft functions required to achieve $\mathrm{N}^{k} \mathrm{LL}$ and $\mathrm{N}^{k} \mathrm{LL}^{\prime}$ accuracy in the cumulant $R(\tau)$. Using this table instead of table 5 at $\mathrm{N}^{k} \mathrm{LL}$ order ensures equivalent accuracy between $R(\tau)$ and $\widetilde{\sigma}(\nu)$ upon Laplace transformation of the former.

Some of the subleading terms still missing in eq. (4.55) to make each term be the exact inverse Laplace transform of the corresponding term in eq. (4.47) can be restored by keeping the $\Gamma$ in the truncated differential operator $1+\Gamma \partial_{\Omega}^{2}$ to higher order. Others can be restored by keeping higher-order terms in the Taylor expansion of $e^{\Gamma \partial_{\Omega}^{2}}$, as in eq. (4.51). All these are legitimate options, equivalent at subleading accuracy. The higher the order to which the expansion of the differential operator $e^{\Gamma \partial_{\Omega}^{2}}$ is kept, the higher the accuracy to which $R\left(\tau_{a}\right)$ computed using eq. (4.43) is in fact the correct inverse Laplace transform of $\widetilde{\sigma}(\nu)$ computed using eq. (4.40). The minimal prescription to maintain equivalence between the accuracy of $R(\tau)$ and $\widetilde{\sigma}(\nu)$ at NLL is to keep $e^{\Gamma \partial_{\Omega}^{2}}$ up to at least the $\Gamma \partial_{\Omega}^{2}$ term of the Taylor expansion, then truncated to $\mathcal{O}\left(\alpha_{s}\right)$ or greater. For $\mathrm{N}^{k} \mathrm{LL}$ accuracy, one keeps the differential operator up to at least the $\Gamma^{k} \partial_{\Omega}^{2 k}$ term of the Taylor expansion, then truncated to $\mathcal{O}\left(\alpha_{s}^{k}\right)$ or greater. This prescription for evaluating $R(\tau)$ to $\mathrm{N}^{k} \mathrm{LL}$ accuracy is given in table 6 . Using table 6 ensures that the Laplace transform of $R(\tau)$ reproduces the logs in the fixed-order expansion of $\widetilde{\sigma}(\nu)$ eq. (4.47) that are fully predicted by exponentiating eq. (4.46) at the same order of accuracy.

Up until now we have not considered the effect of the $C\left(a_{s}\right)$ factor in front of eq. (4.40) or eq. (4.43). The above considerations apply to maintaining equivalent accuracy between the logs of $\tau$ or $\nu$ predicted by expanding the exponentiated logs in $\widetilde{\sigma}(\nu)$ in eq. (4.40) or the exponentials, derivative operators, and gamma function in $R(\tau)$ in eq. (4.43). These terms determine the shape of the distribution. If one multiplies through the expansion of $C\left(a_{s}\right)$ in eq. (4.47) (which affects the normalization), there are terms that are missing at $\mathrm{N}^{k} \mathrm{LL}$ order (with $C\left(a_{s}\right)$ truncated according to table 5 ) that are the same size as those missed by truncating the differential operator $e^{\Gamma \partial_{\Omega}^{2}}$ to the same order. For example, at NLL order, one of the leading effects of the missing $\mathcal{O}\left(a_{s}\right)$ coefficient $C_{1}$ is a missing $a_{s}^{2} C_{1} \Gamma_{0} \tilde{L}^{2}$ term in eq. (4.47). Since this is the same size as the $a_{s}^{2} \tilde{L}^{2}$ term we pointed out would be mispredicted by truncating the differential operator $e^{\Gamma \partial_{\Omega}^{2}}$ to tree level in eq. (4.53), it is fair to complain that one should not demand keeping the differential operator to higher order than the order to which the $C_{n}$ coefficients are known. Thus, it is not incorrect to use table 5 to evaluate all the objects in $R(\tau)$. We are pointing out that, as far as the exponentiation of the logs themselves in eq. (4.40) is concerned, one loses information that is contained in the exponent of eq. (4.40) by Laplace transforming eq. (4.43) with the differential operator truncated according to table 5 instead of table 6 . This is information one already has 
available if one knows the anomalous dimensions needed at $\mathrm{N}^{k} \mathrm{LL}$ accuracy, and so it need not be thrown away. If we separate the counting of the exponentiated logs in eq. (4.40) illustrated in eq. (4.47) from the fixed-order non-log coefficient $C\left(a_{s}\right)$, then table 6 should be used to evaluate $R(\tau)$ in order to preserve the accuracy of the logs predicted in eq. (4.47) upon Laplace transformation.

For these reasons we say it is preferable to compute $R(\tau)$ in momentum space using table 6 instead of table 5 when working to $\mathrm{N}^{k} \mathrm{LL}$ accuracy. And it is even better to work to full $\mathrm{N}^{k} \mathrm{LL}^{\prime}$ accuracy if possible. Then not only does $R(\tau)$ match the accuracy of the $\mathrm{N}^{k} \mathrm{LL}^{\prime}$ Laplace transform $\widetilde{\sigma}(\nu)$, the coefficients $C_{n}$ and terms in the differential operator $e^{\Gamma \partial_{\Omega}^{2}}$ are kept to a consistent accuracy. If the coefficients $C_{n}$ of the non-logarithmic terms required at $\mathrm{N}^{k} \mathrm{LL}^{\prime}$ accuracy are not available, then keeping just the differential operator terms in the jet and soft functions (or the $\partial_{E^{\prime}}^{n}$ terms in the dQCD form) to $\mathrm{N}^{k} \mathrm{LL}^{\prime}$ accuracy in evaluating the cumulant $R\left(\tau_{a}\right)$ is sufficient to maintain equivalent accuracy with the $\mathrm{N}^{k}$ LL Laplace transform $\widetilde{\sigma}\left(\nu_{a}\right)$, in the manner illustrated in eqs. (4.47) and (4.55).

Nota bene: in remainder of the paper, we restore the running of $\alpha_{s}$.

\subsection{Distribution}

The result of taking the inverse Laplace transform of eq. (4.2) is the expression for the resummed distribution $\sigma\left(\tau_{a}\right)$ given in eq. (3.46). For the canonical scale choices eq. (4.25), this simplifies to:

$$
\sigma\left(\tau_{a}\right)=H_{2}(Q) \frac{e^{K}}{\tau_{a}} \widetilde{J}\left(\partial_{\Omega}, \mu_{J}^{\mathrm{nat}}\right)^{2} \widetilde{S}\left(\partial_{\Omega}, \mu_{S}^{\mathrm{nat}}\right)\left[\frac{e^{\gamma_{E} \Omega}}{\Gamma(-\Omega)}\right]
$$

with $K, \Omega$ given by eq. (4.28). Alternatively, for the scale choices eq. (3.81), we obtain the form

$$
\sigma\left(\tau_{a}\right)=H_{2}(Q) \frac{e^{\bar{K}}}{\tau_{a}} \widetilde{J}\left(\partial_{\bar{\Omega}}, \bar{\mu}_{J}\right)^{2} \widetilde{S}\left(\partial_{\bar{\Omega}}, \bar{\mu}_{S}\right)\left[\frac{1}{\Gamma(-\bar{\Omega})}\right]
$$

where $\bar{K}, \bar{\Omega}$ are given by eq. (4.30). These forms for $\sigma\left(\tau_{a}\right)$ correspond to making $\tau_{a^{-}}$ dependent scale choices after differentiating the cumulant $R\left(\tau_{a}\right)$ given by eq. (3.48), which is the clockwise route in figure 2 .

For the distribution $\sigma\left(\tau_{a}\right)$, which is the derivative of the cumulant $R\left(\tau_{a}\right)$, one might expect that the accuracy of logarithmic resummation can be classified in the same way as for $R\left(\tau_{a}\right)$ or for $\widetilde{\sigma}(\nu)$, and thus achieved by calculating the quantities in table 5 (or table 6 ) to the orders specified therein, in particular truncating the fixed-order coefficients $\widetilde{J}, \widetilde{S}$ to the specified accuracy. This is how one might interpret the prescriptions for computing $\sigma\left(\tau_{a}\right)$ using the form eq. (4.58) given in, e.g., [71]. The situation is slightly more subtle, however, and the truncation of $\widetilde{J}, \widetilde{S}$ according to table 5 or table 6 should not yet be performed in eq. (4.58) or eq. (4.59).

Consider the expression in eq. (4.59). Since the series expansion of $\Gamma(-\bar{\Omega})$ about $\bar{\Omega}=0$ starts with $-1 / \bar{\Omega}$, it is not expedient to place it directly into the exponent of the cumulant by taking its $\log$ as in eq. (4.34). Instead, let us pull out a factor of $-\bar{\Omega}$ to turn eq. (4.58) 
into as close a form as possible to the cumulant in eq. (4.27). This results in

$$
\sigma\left(\tau_{a}\right)=-H_{2}(Q) \frac{e^{\bar{K}}}{\tau_{a}} \widetilde{J}\left(\partial_{\bar{\Omega}}\right)^{2} \widetilde{S}\left(\partial_{\bar{\Omega}}\right)\left[\frac{\bar{\Omega}}{\Gamma(1-\bar{\Omega})}\right]
$$

Now pull the factor of $\bar{\Omega}$ through the functions of $\partial_{\bar{\Omega}}$ and relate derivatives of the quantity in brackets to those of $\mathcal{G}(\bar{\Omega})$ given by eq. (4.33):

$$
\begin{aligned}
\mathcal{F}(\bar{\Omega}) & \equiv \bar{\Omega} \mathcal{G}(\bar{\Omega}), \\
\partial_{\bar{\Omega}} \mathcal{F}(\bar{\Omega}) & =\bar{\Omega} \partial_{\bar{\Omega}} \mathcal{G}(\bar{\Omega})+\mathcal{G}(\bar{\Omega}), \\
\partial_{\bar{\Omega}}^{2} \mathcal{F}(\bar{\Omega}) & =\bar{\Omega} \partial_{\bar{\Omega}}^{2} \mathcal{G}(\bar{\Omega})+2 \partial_{\bar{\Omega}} \mathcal{G}(\bar{\Omega}), \\
\partial_{\bar{\Omega}}^{3} \mathcal{F}(\bar{\Omega}) & =\bar{\Omega} \partial_{\bar{\Omega}}^{3} \mathcal{G}(\bar{\Omega})+3 \partial_{\bar{\Omega}}^{2} \mathcal{G}(\bar{\Omega}), \\
\partial_{\bar{\Omega}}^{4} \mathcal{F}(\bar{\Omega}) & =\bar{\Omega} \partial_{\bar{\Omega}}^{4} \mathcal{G}(\bar{\Omega})+4 \partial_{\bar{\Omega}}^{3} \mathcal{G}(\bar{\Omega}),
\end{aligned}
$$

and so on. Thus, the distribution eq. (4.60) can be written very closely to the form of the cumulant eq. (4.29):

$$
\sigma\left(\tau_{a}\right)=-H_{2}(Q) \frac{e^{\bar{K}}}{\tau_{a}}\left[\bar{\Omega} \widetilde{F}\left(\partial_{\bar{\Omega}}\right)+\widetilde{G}\left(\partial_{\bar{\Omega}}\right)\right]\left[\frac{1}{\Gamma(1-\bar{\Omega})}\right],
$$

where

$$
\widetilde{F}\left(\partial_{\Omega}\right)=\widetilde{J}\left(\partial_{\Omega}\right)^{2} \widetilde{S}\left(\partial_{\Omega}\right)
$$

and

$$
\widetilde{G}(L) \equiv \frac{d \widetilde{F}(L)}{d L},
$$

where $L$ is a variable standing in for $\partial_{\bar{\Omega}}$. The extra terms on each line of eq. (4.61) show that $\widetilde{G}$ can be constructed from $\widetilde{F}$ by differentiation with respect to the $\partial_{\bar{\Omega}}$ operator itself.

The form eq. (4.62) is very similar to the cumulant eq. (4.27), but presents a conundrum: how do we deal with the prefactors $\widetilde{F}, \widetilde{G}$ in defining $\mathrm{N}^{k} \mathrm{LL}$ accuracy for $\sigma\left(\tau_{a}\right)$ ? There is now an overall factor of $1 / \tau_{a}$, which should be counted as one log. This promotes the terms in the prefactor $H[\Omega \widetilde{F}+\widetilde{G}]$ to one higher order in log accuracy than in the cumulant. Applying the power counting that $\alpha_{s} \ln \tau \sim 1$, we would conclude that the tree-level terms in $H, \widetilde{F}, \widetilde{G}$ are LL order, the $\mathcal{O}\left(\alpha_{s}\right)$ terms are NLL, $\mathcal{O}\left(\alpha_{s}^{2}\right)$ terms are NNLL, etc. Strictly applying this power counting, even the constant terms $c_{H, J, S}$ in the hard, jet, and soft functions are promoted to one higher order than in the cumulant alone. This is certainly a possible choice of convention, which corresponds to $\mathrm{N}^{k} \mathrm{LL}^{\prime}$ accuracy in table 5 and table 6 . As with the cumulant, working to $\mathrm{N}^{k} \mathrm{LL}$ accuracy in table 6 is preferable to $\mathrm{N}^{k} \mathrm{LL}$ accuracy in table 5 , and $\mathrm{N}^{k} \mathrm{LL}^{\prime}$ accuracy is even better, as it obtains the correct $\mathrm{N}^{k} \mathrm{LL}$ terms as well as the complete $\mathcal{O}\left(\alpha_{s}^{k}\right)$ fixed-order singular terms in the distribution.

We now explore an alternative approach to the distribution, which is to define a prescription so that it equals the derivative of the cumulant at a given order of accuracy, or at least as close to this as possible that we can achieve in practice. 


\subsubsection{Keeping cumulant and distribution to consistent accuracy}

We wish to define the $\mathrm{N}^{k} \mathrm{LL}$ distribution $\sigma\left(\tau_{a}\right)$ by identifying it with the derivative of the $\mathrm{N}^{k}$ LL cumulant $R\left(\tau_{a}\right)$ with respect to $\tau_{a}$, so that integrating the $\mathrm{N}^{k} \mathrm{LL}$ distribution gives back the $\mathrm{N}^{k} \mathrm{LL}$ cumulant. We will find that first resumming the cumulant in SCET using eq. (3.48) and then differentiating it (following the counterclockwise route in figure 2) gives a distribution with a closer correspondence with the direct QCD resummation of the cross section than does using eq. (3.46) (resulting from the clockwise route in figure 2) directly.

It is possible to reorganize the result of following the clockwise route in figure 2 so that at any truncated $\mathrm{N}^{k} \mathrm{LL}$ order it more closely matches the result of following the counterclockwise route. Above, we took the formula eq. (4.59) for the SCET distribution obtained by choosing the scales eq. (3.81) and plugging into eq. (3.46) (clockwise, figure 2), and reorganized its pieces into the form eq. (4.62). This form eq. (4.62) can be truncated in such a way as to correspond to the derivative of the cumulant eq. (4.29) (counterclockwise, figure 2) at $\mathrm{N}^{k} \mathrm{LL}$ accuracy, namely, by truncating the terms in $H, \tilde{J}, \tilde{S}$ in eq. (4.62) according to the usual $\mathrm{N}^{k} \mathrm{LL}$ table 5 , except for the $\widetilde{G}\left(\partial_{\bar{\Omega}}\right)$ term produced by pulling $\bar{\Omega}$ through the $\widetilde{J}^{2}\left(\partial_{\bar{\Omega}}\right) \widetilde{S}\left(\partial_{\bar{\Omega}}\right)$ operators in eq. (4.60). We will find that we must keep a subset of terms in $\widetilde{G}$ to higher order than we do for $\widetilde{F}$ specified in table 5 in order for the $\mathrm{N}^{k} \mathrm{LL}$ distribution to equal the derivative of the $\mathrm{N}^{k} \mathrm{LL}$ cumulant. Truncating $\tilde{J}, \tilde{S}$ in eq. (4.58) according to table 5 before pulling $\bar{\Omega}$ through the differential operators in eq. (4.60) will not produce the derivative of the full $\mathrm{N}^{k} \mathrm{LL}$ radiator but will be missing terms that are in $\widetilde{G}$ in eq. (4.62). Some of these missing terms are in fact required at unprimed $\mathrm{N}^{k} \mathrm{LL}$ accuracy; at $\mathrm{N}^{k} \mathrm{LL}^{\prime}$ accuracy, they are formally subleading, but ensure closer numerical correspondence with the derivative of the cumulant. If full $\mathrm{N}^{k} \mathrm{LL}^{\prime}$ results are not available, the truncation rules for $\mathrm{N}^{k} \mathrm{LL}$ accuracy in table 6 also suffice to make eq. (4.62) match the accuracy of the $\mathrm{N}^{k} \mathrm{LL}$ cumulant.

Since remembering which terms in $\widetilde{G}$ are strictly necessary can be cumbersome, the most straightforward way to compute the differential distribution so that it matches the accuracy of the $\mathrm{N}^{k} \mathrm{LL}$ cumulant is simply to follow the counterclockwise route in figure 2 and differentiate after choosing appropriate $\tau_{a}$-dependent scales. We will derive below a generic formula for the result of this procedure. This procedure guarantees that the resulting distribution $\sigma\left(\tau_{a}\right)$ matches the accuracy of the cumulant $R\left(\tau_{a}\right)$ that we started with.

We note that this approach of differentiating the resummed cumulant to obtain the distribution has been previously considered, and two potential (related) issues have been noted [38]. First, the uncertainties from scale variation may be unreasonably small in the tail region of the distribution, and second, the accuracy of the tail region when matching onto fixed order may be compromised. These complications arise because the tail region of the distribution has large cancellations between singular terms (those participating in the resummation) and nonsingular terms. Care is required when turning off the resummation in the tail region to preserve this delicate cancellation and also produce reliable uncertainties. While these problems will not be solved here, we hope that the approach taken here offers ways to address these issues in the future.

Following the counterclockwise route in figure 2, we take the derivative of the cumulant after setting the scales $\mu_{J, S}$ to be $\tau_{a}$-dependent. For generality, we will allow for arbitrary 
functional dependence $\mu_{J, S}=\mu_{J, S}\left(\tau_{a}\right)$ (generalizing the canonical choices in figure 2). Starting from eq. (3.48) and taking the $\tau_{a}$ derivative:

$$
\begin{aligned}
\frac{d R}{d \tau_{a}}= & \sigma_{n}\left(\tau_{a}\right)+\frac{1}{\tau_{a}} \exp \left(K_{H}+2 K_{J}+K_{S}\right)\left(\frac{\mu_{H}}{Q}\right)^{\omega_{H}}\left(\frac{\mu_{J}}{Q \tau_{a}^{1 / j_{J}}}\right)^{2 j_{J} \omega_{J}}\left(\frac{\mu_{S}}{Q \tau_{a}}\right)^{\omega_{S}} H_{2}\left(Q^{2}, \mu_{H}\right) \\
\times & \left\{\sum _ { F = J _ { n } , J _ { \overline { n } } , S } \frac { d \operatorname { l n } \mu _ { F } } { d \operatorname { l n } \tau _ { a } } \left[\frac{d K}{d \ln \mu_{F}}+j_{F} \omega_{F}+j_{F} \frac{d \omega_{F}}{d \ln \mu_{F}} \ln \frac{\mu_{F}}{Q \tau_{a}^{1 / j_{F}}}\right.\right. \\
& \left.\left.+\frac{d \Omega}{d \ln \mu_{F}} \partial_{\Omega}+\frac{d}{d \ln \mu_{F}} \ln \widetilde{F}\left(\partial_{\Omega}+\ln \frac{\mu_{F}^{j_{F}}}{Q^{j_{F}} \tau_{a}}, \mu_{F}\right)\right]\right\} \\
\times & \widetilde{J}_{n}\left(\partial_{\Omega}+\ln \frac{\mu_{J}^{j_{J}}}{Q^{j_{J}} \tau_{a}}, \mu_{J}\right) \widetilde{J}_{\bar{n}}\left(\partial_{\Omega}+\ln \frac{\mu_{J}^{j_{J}}}{Q^{j_{J}} \tau_{a}}, \mu_{J}\right) \widetilde{S}\left(\partial_{\Omega}+\ln \frac{\mu_{S}}{Q \tau_{a}}, \mu_{S}\right) \frac{\exp \left(\gamma_{E} \Omega\right)}{\Gamma(1-\Omega)} .
\end{aligned}
$$

The spectrum $\sigma_{n}\left(\tau_{a}\right)$ in the first term is the "natural" SCET spectrum, given in eq. (3.46). The jet functions $J_{n}$ and $J_{\bar{n}}$ and their profiles $\mu_{J_{n}}$ and $\mu_{J_{\bar{n}}}$ are treated separately to avoid the combinatoric factors from a common jet function label. Using the relations

$$
\begin{aligned}
\frac{d K}{d \ln \mu_{F}} & =-j_{F} \omega_{F}-\gamma_{F}\left[\alpha_{s}\left(\mu_{F}\right)\right], \\
\frac{d \omega_{F}}{d \ln \mu_{F}} & =-\frac{2}{j_{F}} \Gamma_{F}\left[\alpha_{s}\left(\mu_{F}\right)\right], \\
\frac{d}{d \ln \mu_{F}} \widetilde{F}\left(\partial_{\Omega}+\ln \frac{\mu_{F}^{j_{F}}}{Q^{j_{F}} \tau_{a}}, \mu_{F}\right) & =\left[\beta\left[\alpha_{s}\left(\mu_{F}\right)\right] \frac{\partial \widetilde{F}\left(\mathbf{L}_{F}, \mu_{F}\right)}{\partial \alpha_{s}}+j_{F} \frac{d \widetilde{F}\left(\mathbf{L}_{F}, \mu_{F}\right)}{d \mathbf{L}_{F}}\right], \\
\text { with } \mathbf{L}_{F} & =\partial_{\Omega}+\ln \mu_{F}^{j_{F}} / Q^{j_{F}} \tau_{a},
\end{aligned}
$$

we can write the spectrum $\sigma_{R}$, which is the derivative of the cumulant when working to all orders:

$$
\begin{aligned}
\sigma_{R}\left(\tau_{a}\right)= & \frac{d R}{d \tau_{a}}=\sigma_{n}\left(\tau_{a}\right)+\delta \sigma_{R}\left(\tau_{a}\right), \\
\delta \sigma_{R}\left(\tau_{a}\right)= & \frac{1}{\tau_{a}} \exp \left(K_{H}+2 K_{J}+K_{S}\right)\left(\frac{\mu_{H}}{Q}\right)^{\omega_{H}}\left(\frac{\mu_{J}}{Q \tau_{a}^{1 / j_{J}}}\right)^{2 j_{J} \omega_{J}}\left(\frac{\mu_{S}}{Q \tau_{a}}\right)^{\omega_{S}} H_{2}\left(Q^{2}, \mu_{H}\right) \\
& \times\left\{\sum_{F=J, J, S} \widetilde{P}_{F}\left(\mathbf{L}_{F}, \mu_{F}\right) \frac{1}{\widetilde{F}\left(\mathbf{L}_{F}, \mu_{F}\right)}\right\} \widetilde{J}\left(\mathbf{L}_{J}, \mu_{J}\right)^{2} \widetilde{S}\left(\mathbf{L}_{S}, \mu_{S}\right) \frac{\exp \left(\gamma_{E} \Omega\right)}{\Gamma(1-\Omega)},
\end{aligned}
$$

with $\quad \mathbf{L}_{J}=\partial_{\Omega}+\ln \mu_{J}^{j_{J}} / Q^{j_{J}} \tau_{a}, \quad \mathbf{L}_{S}=\partial_{\Omega}+\ln \mu_{S} / Q \tau_{a}$.

The function $\widetilde{P}_{F}$ is defined

$$
\begin{aligned}
\widetilde{P}_{F}\left(L_{F}, \mu_{F}\right)=\frac{d \ln \mu_{F}}{d \ln \tau_{a}}\left\{j_{F} \frac{\partial \widetilde{F}\left(L_{F}, \mu_{F}\right)}{\partial L_{F}}+\beta\left[\alpha_{s}\left(\mu_{F}\right)\right]\right. & \frac{\partial \widetilde{F}\left(L_{F}, \mu_{F}\right)}{\partial \alpha_{s}} \\
& \left.-\widetilde{\gamma}_{F}\left(L_{F}, \mu_{F}\right) \widetilde{F}\left(L_{F}, \mu_{F}\right)\right\} .
\end{aligned}
$$

The $\widetilde{\gamma}_{F}$ in the last term of eq. (4.68) takes the same form as the usual anomalous dimension in eq. (3.32),

$$
\widetilde{\gamma}_{F}\left(L_{F}, \mu\right) \equiv-\kappa_{F} \Gamma_{\text {cusp }}^{q}\left[\alpha_{s}\right] L_{F}+\gamma_{F}\left[\alpha_{s}\right]
$$


but in eq. (4.67) it becomes a differential operator upon making the replacements $L_{F} \rightarrow \mathbf{L}_{F}$ given in the last line of eq. (4.67). In eq. (4.67), the factor of $1 / \widetilde{F}$ is present to cancel the factor of $\widetilde{F}$ present in $\widetilde{J} \widetilde{S}^{2}$. Note that in this form there is no need to separately label the two jet functions [as there was in eq. (4.65)].

Since table 5 (or table 6 if using the prescription discussed in section 4.2.2) specifies to what accuracy the various ingredients in the cumulant are kept when working to a given order, for $\sigma_{R}$ table 5 or table 6 should also be used to define the orders of resummed accuracy. The fact that the spectrum $\sigma_{n}$ and the derivative of the cumulant are equivalent when working to all orders implies that $\widetilde{P}_{F}$ vanishes if all orders of accuracy are kept. In fact, working to $\mathrm{N}^{k} \mathrm{LL}^{\prime}, \widetilde{P}_{F}$ starts at $\mathcal{O}\left(\alpha_{s}^{k+1}\right)$. [However, working to $\mathrm{N}^{k} \mathrm{LL}, \widetilde{P}_{F}$ starts at $\mathcal{O}\left(\alpha_{s}^{k}\right)$.] Although we have derived $\sigma_{R}$ by differentiating the cumulant, the way in which the truncation occurs at a given finite order of accuracy is different between the cumulant and $\sigma_{R}$. For example, the all-orders evolution kernel $\eta_{\Gamma}$ in eq. (3.34) and cusp anomalous dimension $\Gamma_{\text {cusp }}$ obey the exact relation

$$
\frac{d \eta_{\Gamma}\left(\mu, \mu_{F}\right)}{d \ln \mu_{F}}=-\Gamma_{\mathrm{cusp}}^{q}\left[\alpha_{s}\left(\mu_{F}\right)\right]
$$

but at a finite order of accuracy the function defining $\eta_{\Gamma}$ is truncated in such a way that its derivative differs at the level of higher order terms beyond the requested order of accuracy. For example, at NLL, using eqs. (4.18) and (4.22) and the counting of anomalous dimensions and beta functions terms in table 5 , we find

$$
\frac{d\left(\eta_{\Gamma}^{\mathrm{LL}}+\eta_{\Gamma}^{\mathrm{NLL}}\right)}{d \ln \mu_{F}}=-\frac{\alpha_{s}\left(\mu_{F}\right)}{4 \pi} \Gamma_{0}-\left(\frac{\alpha_{s}\left(\mu_{F}\right)}{4 \pi}\right)^{2} \Gamma_{1}-\left(\frac{\alpha_{s}\left(\mu_{F}\right)}{4 \pi}\right)^{3}\left(\frac{\Gamma_{1} \beta_{1}}{\beta_{0}}-\frac{\Gamma_{0} \beta_{1}^{2}}{\beta_{0}^{2}}\right),
$$

thus with both sides of eq. (4.70) truncated at NLL accuracy, the relation holds only up to subleading terms [here $\mathcal{O}\left(\alpha_{s}^{3}\right)$ ]. These kinds of mismatches, formally subleading, lead to small numerical differences in the values of $\sigma_{R}$ in eq. (4.67) and $d R / d \tau_{a}$ computed by differentiating eq. (3.48) when truncated to a finite accuracy.

The form in eq. (4.67) is fully generic, and simplifies significantly with simple scale choices such that we can compare to the form in eq. (4.62). For the scale choice in eq. (3.81),

$$
\sigma_{R}\left(\tau_{a}\right)=H_{2}(Q) e^{\bar{K}}\left[-\frac{1}{\tau_{a}}\left(\bar{\Omega}+\sum_{F} \frac{1}{j_{F}} \bar{\gamma}_{F}\left(\partial_{\bar{\Omega}}\right)\right) \widetilde{F}\left(\partial_{\bar{\Omega}}\right)+\frac{d \widetilde{F}\left(\partial_{\bar{\Omega}}\right)}{d \tau_{a}}\right] \frac{1}{\Gamma(1-\bar{\Omega})},
$$

where the operator $\bar{\gamma}_{F}\left(\partial_{\bar{\Omega}}\right)$ is defined

$$
\bar{\gamma}_{F}\left(\partial_{\bar{\Omega}}\right)=-\kappa_{F} \Gamma_{\text {cusp }}^{q}\left[\alpha_{s}\left(\bar{\mu}_{F}\right)\right] \partial_{\bar{\Omega}}+\gamma_{F}\left[\alpha_{s}\left(\bar{\mu}_{F}\right)\right],
$$

which is formed from the anomalous dimension eq. (3.32) replacing $L_{F}$ with $\partial_{\bar{\Omega}}$.

If we start with the cumulant $R$ at a given order of $\mathrm{N}^{k} \mathrm{LL}$ accuracy with $\gamma_{F}, \Gamma_{F}, \widetilde{F}$ computed to the orders given in table 5 or table 6 , then eq. (4.72) is the differential distribution at the equivalent order of $\mathrm{N}^{k} \mathrm{LL}$ accuracy, where each piece of eq. (4.72) is truncated to the same order as in $R\left(\tau_{a}\right)$ itself. Suppose, however, we start directly with the formula eq. (4.62) for the distribution $\sigma\left(\tau_{a}\right)$. Note that the first term in brackets of 
each of eqs. (4.62) and (4.72) are the same. Thus the remaining terms in eq. (4.72) must correspond to the terms in the $\widetilde{G}\left(\partial_{\bar{\Omega}}\right)$ operator in eq. (4.62). Note that we must keep the terms in $\widetilde{G}$ to higher order than $\widetilde{F}$ in table 5 or table 6 to keep the two formulae eqs. (4.62) and (4.72) equivalent. For example, using the table 5 prescription for computing to NLL accuracy, we start with the formula eq. (4.72), and keep $\widetilde{F}$ to $\mathcal{O}(1), \gamma_{F}$ to $\mathcal{O}\left(\alpha_{s}\right)$ and $\Gamma_{\text {cusp }}^{q}$ to $\mathcal{O}\left(\alpha_{s}^{2}\right)$. Then we obtain the result

$$
\begin{aligned}
\frac{d R_{\mathrm{NLL}}}{d \tau_{a}}= & -\frac{e^{\bar{K}_{\mathrm{NLL}}}}{\tau_{a}}\left(\bar{\Omega}_{\mathrm{NLL}}+\sum_{F} \frac{\alpha_{s}\left(\bar{\mu}_{F}\right)}{4 \pi} \frac{1}{j_{F}}\left\{\gamma_{F}^{0}-\kappa_{F}\left[\Gamma_{\text {cusp }}^{0}+\Gamma_{\text {cusp }}^{1}\left(\frac{\alpha_{s}\left(\bar{\mu}_{F}\right)}{4 \pi}\right)\right] \partial_{\bar{\Omega}}\right\}\right) \\
& \times \frac{1}{\Gamma\left(1-\bar{\Omega}_{\mathrm{NLL}}\right)} .
\end{aligned}
$$

Meanwhile, from the formula eq. (4.62), applying the rules in table 5 naïvely to $\widetilde{F}$ and $\widetilde{G}$ [that is, to $\widetilde{F}$ already in eq. (4.60)], we would obtain the formula

$$
\sigma\left(\tau_{a}\right)=-\frac{1}{\tau_{a}} e^{\bar{K}} \bar{\Omega}_{\mathrm{NLL}} \frac{1}{\Gamma\left(1-\bar{\Omega}_{\mathrm{NLL}}\right)},
$$

which contains only the first term in parentheses in eq. (4.74). The extra terms in eq. (4.74) are contained as a subset of the terms in the $\widetilde{G}$ operator in eq. (4.62):

$$
\begin{aligned}
\widetilde{G}\left(\partial_{\bar{\Omega}}\right)=\sum_{F} & \left\{\frac{\alpha_{s}\left(\bar{\mu}_{F}\right)}{4 \pi} \frac{1}{j_{F}}\left(-\boldsymbol{\kappa}_{\boldsymbol{F}} \boldsymbol{\Gamma}_{\mathbf{0}} \boldsymbol{\partial}_{\overline{\boldsymbol{\Omega}}}+\gamma_{\boldsymbol{F}}^{\mathbf{0}}\right)+\left(\frac{\alpha_{s}\left(\bar{\mu}_{F}\right)}{4 \pi}\right)^{2}\left[\frac{\left(\kappa_{F} \Gamma_{0}\right)^{2}}{2 j_{F}^{2}} \partial_{\bar{\Omega}}^{3}\right.\right. \\
& -\frac{\kappa_{F} \Gamma_{0}}{2 j_{F}^{2}}\left(3 \gamma_{F}^{0}+2 \beta_{0}\right) \partial_{\bar{\Omega}}^{2}+\frac{1}{j_{F}^{2}}\left(-\boldsymbol{\kappa}_{\boldsymbol{F}} \boldsymbol{j}_{\boldsymbol{F}} \boldsymbol{\Gamma}_{\mathbf{1}}+\left(\gamma_{F}^{0}\right)^{2}+2 \gamma_{F}^{0} \beta_{0}-j_{F} \kappa_{F} c_{F}^{1} \Gamma_{0}\right) \partial_{\bar{\Omega}} \\
& \left.\left.+\frac{1}{j_{F}}\left(\gamma_{F}^{1}+c_{F}^{1} \gamma_{F}^{0}+2 c_{F}^{1} \beta_{0}\right)\right]+\cdots\right\}
\end{aligned}
$$

which is computed using eqs. (4.63) and (4.64) and the expansions of $\widetilde{J}, \widetilde{S}$ to $\mathcal{O}\left(\alpha_{s}^{2}\right)$ given by eq. (4.5). The dots indicate additional $\mathcal{O}\left(\alpha_{s}^{2}\right)$ cross terms and higher order terms in $\alpha_{s}$, and we have indicated in bold where the extra terms in eq. (4.74) are found, namely the $\mathcal{O}\left(\alpha_{s}\right)$ part and the $\Gamma_{1}$ term of the $\mathcal{O}\left(\alpha_{s}^{2}\right)$ part of eq. (4.76). Thus, we see that at least a subset of terms in $\widetilde{G}$ are needed to higher order than those in $\widetilde{F}$ in eq. (4.62) in order to reproduce the result of differentiating the cumulant in eq. (4.74). And these terms are contained in $\widetilde{P}_{F}$ in eq. (4.68) at NLL accuracy.

This pattern continues at higher orders. At unprimed $\mathrm{N}^{k} \mathrm{LL}$ order, the extra terms in $\widetilde{G}$ that are contained in $\widetilde{P}_{F}$ in eq. (4.68) are required to be kept to maintain the same accuracy as the $\mathrm{N}^{k} \mathrm{LL}$ cumulant. At primed $\mathrm{N}^{k} \mathrm{LL}$ orders, the extra terms are subleading - the leading ones are actually captured by the $\sigma_{n}$ term in eq. (4.67), but the additional terms in $\delta \sigma_{R}$ ensure closer numerical equivalence with the derivative of the cumulant.

To recap, our proposed formula eq. (4.67) for computing the differential distribution $\sigma\left(\tau_{a}\right)$ adds to the usual form terms that are necessary to maintain equivalent $\mathrm{N}^{k} \mathrm{LL}$ accuracy with the cumulant or Laplace transform. These terms are not strictly needed at $\mathrm{N}^{k} \mathrm{LL}^{\prime}$ accuracy, where the modifications are subleading. Thus our proposed formula does not differ at leading accuracy from any $\mathrm{N}^{k} \mathrm{LL} \mathrm{L}^{\prime}$ results for differential event shape distributions 
in the literature, e.g. those in $[26,38]$. The extra terms are required when evaluating the distribution directly at unprimed $\mathrm{N}^{k} \mathrm{LL}$ accuracy, using the standard formula (equivalent to $\left.\sigma_{n}\right)$ that appears in, e.g., [26, 71, 84]. Thus they should be added to, e.g., the NLL results in [26]. We note that distributions obtained by differentiation of $\mathrm{N}^{k} \mathrm{LL}$ cumulants do retain the correct accuracy even at unprimed order, as in, e.g., [71, 78, 85, 86]. To ensure that the resummed differential distribution given by eq. (3.46) integrates to the $\mathrm{N}^{k} \mathrm{LL}$ cumulant computed from eq. (3.48), we compute the distribution $\sigma_{R}$ in eq. (4.67). In that formula, each piece including the fixed-order functions $\widetilde{F}$ can be truncated according to the rules in table 5 or table 6 , and then $\sigma_{R}$ will maintain the same accuracy as the corresponding cumulant with the pieces truncated according to the same rules.

\subsubsection{Counting accuracy of cumulant and distribution in dQCD formalism}

We can consider also how to compute the distribution and cumulant from the QCDinspired formalism in section 3.4 to consistent accuracy. We can differentiate the radiator in eq. (3.82) [after the scale choices in eq. (3.81)], or we can differentiate eq. (3.80) first and then plug in the scale choices eq. (3.81). The first procedure gives:

$$
\begin{aligned}
\sigma\left(\tau_{a}\right)= & -\frac{1}{\tau_{a}} \frac{d R}{d \ln \left(1 / \tau_{a}\right)} \\
= & -\frac{1}{\tau_{a}} H_{2}(Q) \widetilde{J}(0, Q)^{2} \widetilde{S}(0, Q) \\
& \times\left[\sum_{m=0}^{\infty} \frac{1}{m !} \bar{E}^{(m+1)} \partial_{\bar{E}^{\prime}}^{m}\right] \exp \left[\bar{E}+\sum_{n=2}^{\infty} \frac{1}{n !} \bar{E}^{(n)} \partial_{\bar{E}^{\prime}}^{n}\right] \frac{1}{\Gamma\left(1-\bar{E}^{\prime}\right)}
\end{aligned}
$$

where the operator in the first set of brackets is formed by differentiating the argument of the exponential. Meanwhile the second procedure gives:

$$
\begin{aligned}
\sigma\left(\tau_{a}\right)= & H_{2}(Q) \widetilde{J}(0, Q)^{2} \widetilde{S}(0, Q) \exp \left[\bar{E}+\sum_{n=2}^{\infty} \frac{1}{n !} \bar{E}^{(n)} \partial_{\bar{E}^{\prime}}^{n}\right] \frac{1}{\tau_{a}} \frac{-\bar{E}^{\prime}}{\Gamma\left(1-\bar{E}^{\prime}\right)} \\
= & -\frac{1}{\tau_{a}} H_{2}(Q) \widetilde{J}(0, Q)^{2} \widetilde{S}(0, Q) \\
& \times\left[\bar{E}^{\prime}+\sum_{m=2}^{\infty} \frac{1}{(m-1) !} \bar{E}^{(m)} \partial_{\bar{E}^{\prime}}^{m-1}\right] \exp \left[\bar{E}+\sum_{n=2}^{\infty} \frac{1}{n !} \bar{E}^{(n)} \partial_{\bar{E}^{\prime}}^{n}\right] \frac{1}{\Gamma\left(1-\bar{E}^{\prime}\right)},
\end{aligned}
$$

where the terms in the first set of brackets are formed by moving the $\bar{E}^{\prime}$ in the numerator on the first line through the differential operator in front of it. The operators summed over $m$ on the last line turn out to be the derivatives of the operators summed over $n$ on the first line with respect to $\partial_{\bar{E}^{\prime}}$ itself. With a change of index $(m \rightarrow m+1)$, we note that eq. (4.78) is precisely equal to eq. (4.77).

In the resummed cumulant eq. (3.82), the number of terms we keep in the differential operator is very simple: keep only $\bar{E}$ at LL, keep up to the $\bar{E}^{\prime \prime}$ term (truncating the exponential derivative at $\left.\mathcal{O}\left(\alpha_{s}\right)\right)$ at NLL, keep up to $\bar{E}^{(3)}$ (truncated at $\mathcal{O}\left(\alpha_{s}^{2}\right)$ ) at NNLL, up to $\bar{E}^{(4)}$ (truncated at $\mathcal{O}\left(\alpha_{s}^{3}\right)$ ) at $\mathrm{N}^{3} \mathrm{LL}$, etc. (This counting, summarized in table 7 , corresponds to the rules in table 6.) The distribution eq. (4.77) given by differentiating 


\begin{tabular}{|c|c|c|c|c|c|}
\hline accuracy & $\Gamma_{F}$ & $\gamma_{F}$ & $\beta$ & $\bar{E}^{(j)}$ & $H, \widetilde{J}, \widetilde{S}$ \\
\hline $\mathrm{LL}$ & $\alpha_{s}$ & 1 & $\alpha_{s}$ & $j=1, \alpha_{s}$ & 1 \\
\hline $\mathrm{NLL}$ & $\alpha_{s}^{2}$ & $\alpha_{s}$ & $\alpha_{s}^{2}$ & $j=2, \alpha_{s}$ & 1 \\
\hline $\mathrm{NNLL}$ & $\alpha_{s}^{3}$ & $\alpha_{s}^{2}$ & $\alpha_{s}^{3}$ & $j=3, \alpha_{s}^{2}$ & $\alpha_{s}$ \\
\hline $\mathrm{N}^{3} \mathrm{LL}$ & $\alpha_{s}^{4}$ & $\alpha_{s}^{3}$ & $\alpha_{s}^{4}$ & $j=4, \alpha_{s}^{3}$ & $\alpha_{s}^{2}$ \\
\hline
\end{tabular}

\begin{tabular}{|c|c|c|c|c|c|}
\hline accuracy & $\Gamma_{F}$ & $\gamma_{F}$ & $\beta$ & $\bar{E}^{(j)}$ & $H, \widetilde{J}, \widetilde{S}$ \\
\hline $\mathrm{LL}$ & $\alpha_{s}$ & 1 & $\alpha_{s}$ & $j=1, \alpha_{s}$ & 1 \\
\hline $\mathrm{NLL}^{\prime}$ & $\alpha_{s}^{2}$ & $\alpha_{s}$ & $\alpha_{s}^{2}$ & $j=2, \alpha_{s}$ & $\alpha_{s}$ \\
\hline $\mathrm{NNLL}^{\prime}$ & $\alpha_{s}^{3}$ & $\alpha_{s}^{2}$ & $\alpha_{s}^{3}$ & $j=3, \alpha_{s}^{2}$ & $\alpha_{s}^{2}$ \\
\hline $\mathrm{N}^{3} \mathrm{LL}^{\prime}$ & $\alpha_{s}^{4}$ & $\alpha_{s}^{3}$ & $\alpha_{s}^{4}$ & $j=4, \alpha_{s}^{3}$ & $\alpha_{s}^{3}$ \\
\hline
\end{tabular}

Table 7. Order of anomalous dimensions, beta function, coefficients $\bar{E}^{(n)}$ of derivative operators, and non-log coefficients in $H, \widetilde{J}, \widetilde{S}$ in dQCD form eq. (3.80) of cumulant $R(\tau)$ required to achieve $\mathrm{N}^{k} \mathrm{LL}$ and $\mathrm{N}^{k} \mathrm{LL} \mathrm{L}^{\prime}$ accuracy. These tables are also applicable to the form for the distribution $\sigma\left(\tau_{a}\right)$ in the last line of eq. (4.78).

that formula then has terms in the sum in the prefactor in brackets up to at $\bar{E}^{\prime \prime}$ at LL, $\bar{E}^{(3)}$ at NLL, $\bar{E}^{(4)}$ at NNLL, etc. To get eq. (4.78) to agree exactly with this result, it does not suffice to truncate the differential operator in the first line according to this same scheme. At NLL, if we truncate the sum starting at $\bar{E}^{(3)}$ entirely from the start, we will be missing the $\bar{E}^{(3)}$ term in the last line of eq. (4.78) which is present in the last line of eq. (4.77) if we start with the NLL $R\left(\tau_{a}\right)$ in the first line. The similar mismatch occurs at higher $\mathrm{N}^{k} \mathrm{LL}$ orders. The mismatched terms are formally of subleading order. But if one is interested in keeping the derivative of the cumulant and the directly computed differential distribution in eqs. (4.77) and (4.78) numerically equal to each other, then, similarly to the SCET formalism in the section above, one should keep an extra term in the sum over derivatives in the first set of brackets in the last line of eq. (4.78) than specified by table 7 .

\section{$5 \quad$ Final formulae and prescriptions for $\mathrm{N}^{k} \mathrm{LL}\left({ }^{\prime}\right)$ accuracy}

In this section, we collect in summary form the formulae that can be used to obtain the Laplace transform, cumulant, and distribution at a consistent order of $\mathrm{N}^{k} \mathrm{LL}$ accuracy, in both SCET and QCD-inspired forms. These results follow from the detailed discussion in section 4 .

The standard counting rules in table 5 for achieving $\mathrm{N}^{k} \mathrm{LL}$ or $\mathrm{N}^{k} \mathrm{LL}^{\prime}$ accuracy apply to the formulae eqs. (5.1) and (5.2) for $\widetilde{\sigma}(\nu)$ below. The terms resummed in perturbation theory by computing these formulae according to the rules in these tables form our baseline definition for these orders of accuracy. Our prescriptions for computing $R\left(\tau_{a}\right)$ or $\sigma\left(\tau_{a}\right)$ are motivated by the requirement that they reproduce the accuracy of $\widetilde{\sigma}(\nu)$ computed in this way, upon Laplace transformation.

For the cumulant we give two forms, in SCET and dQCD-like notation, in eqs. (5.4) and (5.5). Our proof in section 3 of the equivalence of these forms is a central technical result of this paper. Thus the dQCD-inspired form eq. (5.5) below can actually be viewed as a SCET form as well, using the definitions eqs. (3.73) and (3.78) for the exponent. The rules in table 6 , in which derivative operator terms in $R\left(\tau_{a}\right)$ are kept to one higher order in $\alpha_{s}$ than implied by table 5, ensure that the Laplace transform of $R\left(\tau_{a}\right)$ reproduces the result of computing $\tilde{\sigma}(\nu)$ in eq. (5.1) or eq. (5.2) according to the rules in table 5, order by 
order in $\alpha_{s}$ to the accuracy illustrated in section 4.2.2. Eq. (5.5) is the first time to our knowledge that a dQCD form for $R\left(\tau_{a}\right)$ with variable jet and soft scales and with derivative operator terms up to arbitrarily high order has been given (see [77] for a similar form with fixed scales in the context of threshold resummation).

For the differential distribution $\sigma\left(\tau_{a}\right)$, our final result, which we call $\sigma_{R}$, is eq. (5.10). In this form, it is safe to truncate the ingredients according to the same rules as the cumulant $R\left(\tau_{a}\right)$ in table 6. We write $\sigma_{R}$ as a sum of two terms $\sigma_{n}+\delta \sigma_{R}$, where the first piece $\sigma_{n}$ is the usual way eq. (3.46) the SCET differential distribution is written, as in $[26,71,84]$. We illustrated in section 4.3 that applying the truncation rules for $\mathrm{N}^{k} \mathrm{LL}$ accuracy in table 5 or table 6 directly to $\sigma_{n}$ would yield a result less accurate than taking the derivative of $R\left(\tau_{a}\right)$ computed at the same accuracy. At $\mathrm{N}^{k} L L^{\prime}$ order, the accuracy of $\sigma_{n}$ or $\sigma_{R}$ is formally the same, but the integral of $\sigma_{R}$ numerically matches the cumulant better. (We note the final results in [71] were computed in terms of the cumulant, not $\sigma(\tau)$, so those results do not suffer from these issues.) The extra term $\delta \sigma_{R}$ restores the missing pieces. Our formula eq. (5.10) is to our knowledge the first time a generic formula for the resummed differential distribution $\sigma\left(\tau_{a}\right)$ that possesses this automatic equivalence to $d R / d \tau_{a}$ has been written down.

It would be instructive to perform similar analyses and comparisons for the event shape resummation performed in [38] using the formalism of [76]; an exercise that nevertheless lies outside the scope of the present paper.

In the following subsections, those equations which are boxed represent our final forms for the Laplace transform, cumulant, and differential distribution, which exhibit full dependence on the hard, jet, and soft scales $\mu_{H, J, S}$ and which are also written in a form where truncation of the ingredients according to the appropriate table in the text will preserve the resummed logarithmic accuracy of the expression, as defined by equivalence to the accuracy of $\tilde{\sigma}(\nu)$.

\subsection{Laplace transform}

For the Laplace transform $\widetilde{\sigma}(\nu)$, eq. (4.2) gives with variable scales, in standard SCET form,

$$
\begin{aligned}
\widetilde{\sigma}(\nu)= & e^{K} H_{2}\left(Q^{2}, \mu_{H}\right)\left(\frac{\mu_{H}}{Q}\right)^{\omega_{H}\left(\mu, \mu_{H}\right)}\left(\frac{\mu_{J} \nu^{1 / j_{J}}}{Q}\right)^{2 j_{J} \omega_{J}\left(\mu_{,} \mu_{J}\right)}\left(\frac{\mu_{S} \nu}{Q}\right)^{\omega_{S}\left(\mu, \mu_{S}\right)-\Omega} \\
& \times \widetilde{J}\left(\partial_{\Omega}+\ln \frac{\mu_{J}^{j_{J}}}{Q^{j_{J}-1} \mu_{S}}, \mu_{J}\right)^{2} \widetilde{S}\left(\partial_{\Omega}, \mu_{S}\right)\left(\frac{\mu_{S} e^{\gamma_{E}} \nu}{Q}\right)^{\Omega}
\end{aligned}
$$

where $K, \Omega$ are defined in eq. (4.3) and $\omega_{F}$ in eq. (3.36). $H_{2}$ is the hard function appearing in the factorization theorem eq. (3.2), and $\widetilde{J}, \widetilde{S}$ are the Laplace transforms of the jet and soft functions $J, S$ appearing therein. $H_{2}$ is given to $\mathcal{O}\left(\alpha_{s}\right)$ in SCET by eq. (3.17), and the momentum space jet and soft functions by eqs. (3.18) and (3.20). The generic definitions of $J, S$ are given in eqs. (3.12) and (3.15). 
In the dQCD-inspired form that was shown in section 3.4 to be equivalent to SCET with the identification in eqs. (3.73) and (3.78), we have, by transforming eq. (3.80),

$$
\begin{aligned}
\widetilde{\sigma}(\nu)= & H_{2}\left(Q^{2}, \mu_{H}\right)\left(\frac{\mu_{H}}{Q}\right)^{\omega_{H}\left(\mu, \mu_{H}\right)} e^{K_{H}\left(\mu, \mu_{H}\right)} \widetilde{J}(0, \mu)^{2} \widetilde{S}(0, \mu) \\
& \times e^{2 E_{J}\left(\mu, \mu_{J}\right)+E_{S}\left(\mu, \mu_{S}\right)} \exp \left[\sum_{n=2}^{\infty} \frac{1}{n !}\left(2 E_{J}^{(n)}\left(\mu_{J}\right) \partial_{2 E_{J}^{\prime}}^{n}+E_{S}^{(n)}\left(\mu_{S}\right) \partial_{E_{S}^{\prime}}^{n}\right)\right] \\
& \times\left(\frac{Q}{\mu_{J}\left(e^{\gamma_{E}} \nu\right)^{1 / j_{J}}}\right)^{2 E_{J}^{\prime}\left(\mu, \mu_{J}\right)}\left(\frac{Q}{\mu_{S} e^{\gamma_{E}} \nu}\right)^{E_{S}^{\prime}\left(\mu, \mu_{S}\right)} .
\end{aligned}
$$

With the scale choices $\mu=\mu_{H}=Q, \mu_{F}=\widetilde{\mu}_{F} \equiv Q\left(e^{\gamma_{E}} \nu\right)^{-1 / j_{F}}$ given in eq. (4.7), the above expressions for $\widetilde{\sigma}(\nu)$ simplify considerably,

$$
\begin{aligned}
\widetilde{\sigma}(\nu) & =H_{2}(Q) \widetilde{J}\left(0, \widetilde{\mu}_{J}\right)^{2} \widetilde{S}\left(0, \widetilde{\mu}_{S}\right) e^{K} \\
& =H_{2}(Q) \widetilde{J}(0, Q)^{2} \widetilde{S}(0, Q) e^{\bar{E}}
\end{aligned}
$$

where here $K=2 K_{J}\left(Q, \widetilde{\mu}_{J}\right)+K_{S}\left(Q, \widetilde{\mu}_{S}\right)$, and $\bar{E}=2 \bar{E}_{J}+\bar{E}_{S}$ is defined in eq. (3.83). The derivatives $E_{J, S}^{(n)}$ are defined in eqs. (3.74) and (3.78). As noted after eq. (3.73), the difference between the exponents $K$ and $\bar{E}$ is NNLL. These differences at $\mathrm{N}^{k}$ LL accuracy, $k \geq 2$, are made up by differences due the scales in the jet and soft functions, which differ in the two lines of eq. (5.3), calculated to the order appropriate to the accuracy in question (see table 5).

Nota bene: the primary definition of $\mathrm{N}^{k} \mathrm{LL}$ or $\mathrm{N}^{k} \mathrm{LL}$ accuracy is based on the accuracy of the exponents in the simple exponentiated forms in eq. (5.3). These accuracies can be achieved by computing ingredients according to table 5 . The theoretical uncertainty at finite resummed $\mathrm{N}^{k} \mathrm{LL}$ accuracy can be estimated by varying the scales in eqs. (5.1) and (5.2).

\subsection{Cumulant}

For the cumulant, the usual resummed form given in SCET with variable scales is given by eq. (3.48),

$$
\begin{aligned}
R\left(\tau_{a}\right)= & e^{K}\left(\frac{\mu_{H}}{Q}\right)^{\omega_{H}}\left(\frac{\mu_{J}}{Q \tau_{a}^{1 / j_{J}}}\right)^{2 j_{J} \omega_{J}}\left(\frac{\mu_{S}}{Q \tau_{a}}\right)^{\omega_{S}} \\
& \times H_{2}\left(Q^{2}, \mu_{H}\right) \widetilde{J}\left(\partial_{\Omega}+\ln \frac{\mu_{J}^{j_{J}}}{Q^{j_{J}} \tau_{a}}, \mu_{J}\right)^{2} \widetilde{S}\left(\partial_{\Omega}+\ln \frac{\mu_{S}}{Q \tau_{a}}, \mu_{S}\right) \frac{\exp \left(\gamma_{E} \Omega\right)}{\Gamma(1-\Omega)}
\end{aligned}
$$

where $K, \Omega$ are defined in eq. (4.3) and $\omega_{F}$ in eq. (3.36). The counting rules in table 6 for computing to $\mathrm{N}^{k} \mathrm{LL}$ or $\mathrm{N}^{k} \mathrm{LL}^{\prime}$ accuracy apply to eq. (5.4). See also the note below. 
Meanwhile the dQCD-inspired form, shown in section 3.4 to be equivalent to SCET, is given by eq. (3.80),

$$
\begin{aligned}
R\left(\tau_{a}\right)= & H_{2}\left(Q^{2}, \mu_{H}\right)\left(\frac{\mu_{H}}{Q}\right)^{\omega_{H}\left(\mu, \mu_{H}\right)} e^{K_{H}\left(\mu, \mu_{H}\right)} \widetilde{J}(0, \mu)^{2} \widetilde{S}(0, \mu) \\
& \times e^{2 E_{J}\left(\mu, \mu_{J}\right)+E_{S}\left(\mu, \mu_{S}\right)} \exp \left[\sum_{n=2}^{\infty} \frac{1}{n !}\left(2 E_{J}^{(n)}\left(\mu_{J}\right) \partial_{2 E_{J}^{\prime}}^{n}+E_{S}^{(n)}\left(\mu_{S}\right) \partial_{E_{S}^{\prime}}^{n}\right)\right] \\
& \times\left(\frac{Q^{j_{J}} \tau_{a}}{\mu_{J}^{j_{J}} e^{\gamma_{E}}}\right)^{2 E_{J}^{\prime}\left(\mu, \mu_{J}\right) / j_{J}}\left(\frac{Q \tau_{a}}{\mu_{S} e^{\gamma_{E}}}\right)^{E_{S}^{\prime}\left(\mu, \mu_{S}\right)} \frac{1}{\Gamma\left(1+2 E_{J}^{\prime} / j_{j}+E_{S}^{\prime}\right)}
\end{aligned}
$$

A closely related form was derived in the context of threshold resummation in ref. [77]. The counting rules in table 7 apply to eq. (5.5). Again the functions $E_{J, S}$ are defined in eqs. (3.73) and (3.78), and the derivatives $E_{J, S}^{(n)}$ in eqs. (3.74) and (3.78).

Choosing the scales in eq. (3.81), $\mu=\mu_{H}=Q, \mu_{F}=\bar{\mu}_{F} \equiv Q\left(e^{-\gamma_{E}} \tau_{a}\right)^{1 / j_{F}}$, we obtained for the above forms,

$$
\begin{aligned}
R\left(\tau_{a}\right) & =H_{2}(Q) e^{\bar{K}} \widetilde{J}\left(\partial_{\bar{\Omega}}, \bar{\mu}_{J}\right)^{2} \widetilde{S}\left(\partial_{\bar{\Omega}}, \bar{\mu}_{S}\right)\left[\frac{1}{\Gamma(1-\bar{\Omega})}\right] \\
& =H_{2}(Q) \widetilde{J}(0, Q)^{2} \widetilde{S}(0, Q) \exp \left[\bar{E}+\sum_{n=2}^{\infty} \frac{1}{n !} \bar{E}^{(n)} \partial_{\bar{E}^{\prime}}^{n}\right] \frac{1}{\Gamma\left(1-\bar{E}^{\prime}\right)},
\end{aligned}
$$

where $\bar{K}, \bar{\Omega}$ are defined in eq. (4.30), and $\bar{E}, \bar{E}^{(n)}$ in eq. (3.83). The first line is the SCET form, eq. (4.29), and the second line follows directly from eq. (5.5). The sign change in the argument of the gamma function between eqs. (5.5) and (5.6) is due to switching taking derivatives with respect to $\ln \mu_{F}$ in the former and $\ln \left(1 / \tau_{a}\right)$ in the latter. These scale choices make the parallel between SCET and QCD forms, as given in previous literature, most transparent. As we will discuss in section 6 , however, it is often preferable to use the "canonical" scales $\mu=\mu_{H}=Q, \mu_{F}^{\text {nat }} \equiv Q \tau_{a}^{1 / j_{F}}$. In this case, the resummed cumulant takes the form

$$
\begin{aligned}
R\left(\tau_{a}\right) & =H_{2}(Q) e^{K} \widetilde{J}\left(\partial_{\Omega}, \mu_{J}^{\text {nat }}\right)^{2} \widetilde{S}\left(\partial_{\Omega}, \mu_{S}^{\text {nat }}\right)\left[\frac{e^{\gamma_{E} \Omega}}{\Gamma(1-\Omega)}\right] \\
& =H_{2}(Q) \widetilde{J}(0, Q)^{2} \widetilde{S}(0, Q) \exp \left[E+\sum_{n=2}^{\infty} \frac{1}{n !} E^{(n)} \partial_{E^{\prime}}^{n}\right] \frac{e^{\gamma_{E} E^{\prime}}}{\Gamma\left(1-E^{\prime}\right)}
\end{aligned}
$$

where the $K, \Omega$ here are defined in eq. (4.28), and $E, E^{(n)}$ are defined by

$$
E=2 E_{J}\left(Q, Q \tau_{a}^{1 / j_{J}}\right)+E_{S}\left(Q, Q \tau_{a}\right), \quad E^{(n)}=\frac{d^{n} E}{d\left(\ln \left(1 / \tau_{a}\right)\right)^{n}},
$$

$E_{J, S}$ being defined by eqs. (3.73) and (3.78).

The dQCD-inspired " $E$ " forms in eqs. (5.6) and (5.7) in fact have SCET definitions by way of the relations in eqs. (3.73), (3.78), and (5.8) (see also eqs. (3.84) and (3.85)). They resum a larger set of terms than the $\Omega$ terms thanks to more logs generated by $\widetilde{J}^{2} \widetilde{S}$ being put in the exponent and gamma functions. 
Nota bene: while table 5 could be used to evaluate the ingredients in the above formulae for $R\left(\tau_{a}\right)$ to $\mathrm{N}^{k} \mathrm{LL}$ accuracy, as explained in section 4.2 .2 it is preferable to keep the differential operator terms in $\widetilde{J}\left(\partial_{\bar{\Omega}}\right), \widetilde{S}\left(\partial_{\bar{\Omega}}\right)$ or the $\partial_{\bar{E}^{\prime}}$ operators to the order corresponding to $\mathrm{N}^{k} L L^{\prime}$ accuracy, as described in table 6 . This maintains better equivalence with the accuracy of the $\mathrm{N}^{k} \mathrm{LL} \tilde{\sigma}(\nu)$. Similarly in eq. (5.7). Working to full $\mathrm{N}^{k} \mathrm{LL}^{\prime}$ accuracy as given by table 5 or table 6 is the ideal. These rules for $\partial_{\Omega}$ terms also apply to evaluation and truncation of the exponential of $\partial_{E^{\prime}}^{n}$ operators in the dQCD forms above, which is summarized in table 7 .

\subsection{Differential distribution}

For the cumulant, a common prescription is the form in eq. (3.46), which we label $\sigma_{n}$ :

$$
\begin{aligned}
\sigma_{n}\left(\tau_{a}\right)= & \exp \left(K_{H}+2 K_{J}+K_{S}\right)\left(\frac{\mu_{H}}{Q}\right)^{\omega_{H}}\left(\frac{\mu_{J}}{Q \tau_{a}^{1 / j_{J}}}\right)^{2 j_{J} \omega_{J}}\left(\frac{\mu_{S}}{Q \tau_{a}}\right)^{\omega_{S}} H_{2}\left(Q^{2}, \mu_{H}\right) \\
& \times \widetilde{J}\left(\partial_{\Omega}+\ln \frac{\mu_{J}^{j_{J}}}{Q^{j_{J}} \tau_{a}}, \mu_{J}\right)^{2} \widetilde{S}\left(\partial_{\Omega}+\ln \frac{\mu_{S}}{Q \tau_{a}}, \mu_{S}\right) \frac{1}{\tau_{a}} \frac{\exp \left(\gamma_{E} \Omega\right)}{\Gamma(-\Omega)} .
\end{aligned}
$$

Often, the rules in table 5 are used to compute $\sigma_{n}$ to a given accuracy. To achieve the same accuracy as the Laplace transform or cumulant at $\mathrm{N}^{k} \mathrm{LL}$ order, it is necessary to use the rules in table 6. At $\mathrm{N}^{k} \mathrm{LL}^{\prime}$ order the two tables are the same, and $\sigma_{n}\left(\tau_{a}\right)$ matches the accuracy of $\widetilde{\sigma}(\nu)$.

An alternate approach is to define a distribution that will reproduce the derivative of the cumulant at any accuracy. This distribution is labeled $\sigma_{R}\left(\tau_{a}\right)$, with [eq. (4.67)]:

$$
\begin{aligned}
\sigma_{R}\left(\tau_{a}\right)= & \sigma_{n}\left(\tau_{a}\right)+\delta \sigma_{R}\left(\tau_{a}\right) \\
\delta \sigma_{R}\left(\tau_{a}\right)= & \frac{1}{\tau_{a}} \exp \left(K_{H}+2 K_{J}+K_{S}\right)\left(\frac{\mu_{H}}{Q}\right)^{\omega_{H}}\left(\frac{\mu_{J}}{Q \tau_{a}^{1 / j_{J}}}\right)^{2 j_{J} \omega_{J}}\left(\frac{\mu_{S}}{Q \tau_{a}}\right)^{\omega_{S}} \\
& \times H_{2}\left(Q^{2}, \mu_{H}\right)\left\{\sum_{F=J, J, S} \widetilde{P}_{F}\left(\mathbf{L}_{F}, \mu_{F}\right) \frac{1}{\widetilde{F}\left(\mathbf{L}_{F}, \mu_{F}\right)}\right\} \\
& \times \widetilde{J}\left(\mathbf{L}_{J}, \mu_{J}\right)^{2} \widetilde{S}\left(\mathbf{L}_{S}, \mu_{S}\right) \frac{\exp \left(\gamma_{E} \Omega\right)}{\Gamma(1-\Omega)} \\
\text { with } \quad \mathbf{L}_{J}= & \partial_{\Omega}+\ln \mu_{J}^{j_{J}} / Q^{j_{J}} \tau_{a}, \quad \mathbf{L}_{S}=\partial_{\Omega}+\ln \mu_{S} / Q \tau_{a},
\end{aligned}
$$

and [eq. (4.68)]:

$$
\begin{aligned}
\widetilde{P}_{F}\left(L_{F}, \mu_{F}\right)=\frac{d \ln \mu_{F}}{d \ln \tau_{a}}\left\{j_{F} \frac{\partial \widetilde{F}\left(L_{F}, \mu_{F}\right)}{\partial L_{F}}+\beta\left[\alpha_{s}\left(\mu_{F}\right)\right] \frac{\partial \widetilde{F}\left(L_{F}, \mu_{F}\right)}{\partial \alpha_{s}}\right. & \\
& \left.-\widetilde{\gamma}_{F}\left(L_{F}, \mu_{F}\right) \widetilde{F}\left(L_{F}, \mu_{F}\right)\right\} .
\end{aligned}
$$

In the form eq. (5.10), $\sigma_{R}\left(\tau_{a}\right)$ can be evaluated using the rules in table 5 or table 6 to achieve $\mathrm{N}^{k} \mathrm{LL}$ or $\mathrm{N}^{k} \mathrm{LL}^{\prime}$ accuracy. Using the form eq. (5.10) will guarantee that equivalent accuracy is maintained with differentiating the $\mathrm{N}^{k} \mathrm{LL}$ or $\mathrm{N}^{k} \mathrm{LL}^{\prime}$ cumulant $R\left(\tau_{a}\right)$, evaluated according the same rules. 
The dQCD-inspired differential cross section, found by taking the derivative of eq. (5.5), can be written with free scales in the form,

$$
\begin{aligned}
\sigma\left(\tau_{a}\right)= & \frac{1}{\tau_{a}} H_{2}\left(Q^{2}, \mu_{H}\right)\left(\frac{\mu_{H}}{Q}\right)^{\omega_{H}\left(\mu, \mu_{H}\right)} e^{K_{H}\left(\mu, \mu_{H}\right)} \widetilde{J}(0, \mu)^{2} \widetilde{S}(0, \mu) \\
& \times e^{2 E_{J}\left(\mu, \mu_{J}\right)+E_{S}\left(\mu, \mu_{S}\right)} \exp \left[\sum_{n=2}^{\infty} \frac{1}{n !}\left(2 E_{J}^{(n)}\left(\mu_{J}\right) \partial_{2 E_{J}^{\prime}}^{n}+E_{S}^{(n)}\left(\mu_{S}\right) \partial_{E_{S}^{\prime}}^{n}\right)\right] \\
& \times\left(\frac{Q^{j_{J}} \tau_{a}}{\mu_{J}^{j_{J}} e^{\gamma_{E}}}\right)^{2 E_{J}^{\prime}\left(\mu, \mu_{J}\right) / j_{J}}\left(\frac{Q \tau_{a}}{\mu_{S} e^{\gamma_{E}}}\right)^{E_{S}^{\prime}\left(\mu, \mu_{S}\right)} \frac{1}{\Gamma\left(2 E_{J}^{\prime} / j_{j}+E_{S}^{\prime}\right)} .
\end{aligned}
$$

Alternatively one may also differentiate eq. (5.5) after choosing $\tau_{a}$-dependent scales, which allows one to apply the counting rules in table 7 directly.

Upon making the particular choices of scales in eq. (3.81), we obtained in SCET and dQCD-inspired forms [eqs. (4.72) and (4.77), respectively],

$$
\begin{aligned}
\sigma\left(\tau_{a}\right)= & H_{2}(Q) e^{\bar{K}}\left[-\frac{1}{\tau_{a}}\left(\bar{\Omega}+\sum_{F} \frac{1}{j_{F}} \bar{\gamma}_{F}\left(\partial_{\bar{\Omega}}\right)\right) \widetilde{F}\left(\partial_{\bar{\Omega}}\right)+\frac{d \widetilde{F}\left(\partial_{\bar{\Omega}}\right)}{d \tau_{a}}\right] \frac{1}{\Gamma(1-\bar{\Omega})} \\
= & -\frac{1}{\tau_{a}} H_{2}\left(Q^{2}, Q\right) \widetilde{J}(0, Q)^{2} \widetilde{S}(0, Q) \\
& \times\left[\sum_{n=0}^{\infty} \frac{1}{n !} \bar{E}^{(n+1)} \partial_{\bar{E}^{\prime}}^{n}\right] \exp \left[\bar{E}+\sum_{n=2}^{\infty} \frac{1}{n !} \bar{E}^{(n)} \partial_{\bar{E}^{\prime}}^{n}\right] \frac{1}{\Gamma\left(1-\bar{E}^{\prime}\right)} .
\end{aligned}
$$

and similarly with the canonical scale choices eq. (4.25),

$$
\begin{aligned}
\sigma\left(\tau_{a}\right)= & H_{2}(Q) e^{K}\left[-\frac{1}{\tau_{a}}\left(\Omega+\sum_{F} \frac{1}{j_{F}} \hat{\gamma}_{F}\left(\partial_{\Omega}\right)\right) \widetilde{F}\left(\partial_{\Omega}\right)+\frac{d \widetilde{F}\left(\partial_{\Omega}\right)}{d \tau_{a}}\right] \frac{e^{\gamma_{E} \Omega}}{\Gamma(1-\Omega)} \\
= & -\frac{1}{\tau_{a}} H_{2}\left(Q^{2}, Q\right) \widetilde{J}(0, Q)^{2} \widetilde{S}(0, Q) \\
& \times\left[\sum_{n=0}^{\infty} \frac{1}{n !} E^{(n+1)} \partial_{E^{\prime}}^{n}\right] \exp \left[E+\sum_{n=2}^{\infty} \frac{1}{n !} E^{(n)} \partial_{E^{\prime}}^{n}\right] \frac{e^{\gamma_{E} E^{\prime}}}{\Gamma\left(1-E^{\prime}\right)}
\end{aligned}
$$

In these expressions the operator $\bar{\gamma}_{F}$ was defined in eq. (4.73), and $\hat{\gamma}_{F}$ is given by the same formula with scales $\mu_{F}$ in eq. (4.25). The exponents $\bar{K}, \bar{\Omega}$ are defined in eq. (4.30), and $K, \Omega$ by eq. (4.28). The exponents $\bar{E}, \bar{E}^{(n)}$ are given by eq. (3.83), and $E, E^{(n)}$ by eq. (5.8). In these forms, one may directly apply the counting rules in table 5 or table 6 for the SCET forms, and table 7 for the dQCD-inspired forms.

Nota bene: use the forms in eq. (5.13) or eq. (5.14) keeping all objects to orders specified in table 5 or table 6 at $\mathrm{N}^{k} \mathrm{LL}$ or $\mathrm{N}^{k} \mathrm{LL}^{\prime}$ accuracy to maintain equivalence with accuracy of $R\left(\tau_{a}\right)$. Do not apply $\mathrm{N}^{k} \mathrm{LL}$ rules in table 5 or table 6 directly to eq. (4.60).

\section{Numerical comparison of angularity distributions}

A numerical study is useful to compare the various resummation prescriptions and the relationship between the dQCD and SCET formalisms. For different values of the angularity parameter $a$, we will study the effect of different prescriptions on NLL and NLL' 
distributions in both SCET and dQCD formalisms, for both the resummed cumulant and spectrum. These prescriptions lead to notable differences between the resummed cross sections, and we will find that the best agreement between SCET and dQCD results are in the most consistent versions of the resummation formulae.

For the resummed cross sections in SCET and dQCD, we use the distributions in eqs. (5.9), (5.10), and (5.12) to NLL and NLL' accuracy. We label the dQCD-inspired form in eq. (5.12) as $\sigma_{Q}\left(\tau_{a}\right)$ for clarity. This study will allow us to contrast the standard SCET resummed distribution, $\sigma_{n}$ [eq. (5.9)], with the SCET resummed form that matches closely with the derivative of the cumulant, $\sigma_{R}$ [eq. (5.10)], as well as the dQCD-inspired form $\sigma_{Q}$ [eq. (5.12)]. The NLL and NLL' distributions for the SCET resummed forms $\sigma_{n}$ and $\sigma_{R}$ are obtained from eqs. (5.9) and (5.10) by applying the counting rules ${ }^{5}$ in table 5 . The NLL and NLL' distributions for the dQCD-inspired form $\sigma_{Q}$ is obtained from eq. (5.12) using the counting prescription described in section 4.3.2. For the case of NLL or NLL' accuracy, this means that eq. (5.12) takes the form

$$
\begin{aligned}
\sigma_{Q}\left(\tau_{a}\right)=- & \frac{1}{\tau_{a}} e^{K_{H}+2 E_{J}+E_{S}} H_{2}\left(Q^{2}, \mu_{H}\right) \widetilde{J}(0, \mu)^{2} \widetilde{S}(0, \mu) \times \\
& \times\left(\frac{\mu_{H}}{Q}\right)^{\omega_{H}}\left(\frac{Q^{j_{J}} \tau_{a}}{\mu_{J}^{j_{J}}}\right)^{2 E_{J}^{\prime} / j_{J}}\left(\frac{Q \tau_{a}}{\mu_{S}}\right)^{E_{S}^{\prime}} \\
& \times\left\{E^{\prime}+2 E_{J}^{\prime \prime}\left(\partial_{E^{\prime}}+\ln \frac{\mu_{J}^{j_{J}}}{Q^{j_{J}} \tau_{a}}\right)+E_{S}^{\prime \prime}\left(\partial_{E^{\prime}}+\ln \frac{\mu_{S}}{Q \tau_{a}}\right)\right. \\
& \left.+E^{\prime} E_{J}^{\prime \prime}\left(\partial_{E^{\prime}}+\ln \frac{\mu_{J}^{j_{J}}}{Q^{j_{J}} \tau_{a}}\right)^{2}+\frac{1}{2} E^{\prime} E_{S}^{\prime \prime}\left(\partial_{E^{\prime}}+\ln \frac{\mu_{S}}{Q \tau_{a}}\right)^{2}\right\} \frac{\exp \left(\gamma_{E} E^{\prime}\right)}{\Gamma\left(1-E^{\prime}\right)} .
\end{aligned}
$$

where $E^{\prime}=-2 E_{J}^{\prime} / j_{J}-E_{S}^{\prime}$. This form arises from eq. (5.12) by expanding the exponentiated derivative operator to $\mathcal{O}\left(\alpha_{s}\right)$, which involves keeping only the leading nontrivial $E_{J}^{\prime \prime}$ and $E_{S}^{\prime \prime}$ terms in the expansion. We also pulled several factors through the derivative operator, as in previous forms.

The numerical study is performed at a center of mass energy of $Q=100 \mathrm{GeV}$, and we make canonical scale choices, eq. (4.25), for the central scales of each distribution. Uncertainty estimates are made through scale variation of $\mu_{H}=\mu, \mu_{J}$, and $\mu_{S}$ each up and down by a factor of 2 . The envelope of these scale variations determines the overall uncertainty. The uncertainty estimates here should be taken as nominal; as we will see, the uncertainties for $\sigma_{R}$ and $\sigma_{Q}$ are not robust (being either overestimated or underestimated in certain regions of $\tau_{a}$ with the scale variations used here) and require further study. Ideally more refined scale variations using parameters in the profile functions themselves should be performed, as in e.g. [38, 76, 78, 79]. We leave such an improved study of the uncertainties for future work. Here, our focus is on the change in behavior of the central values of the curves amongst $\sigma_{n, R, Q}$ and how well they agree with one another. Since there

\footnotetext{
${ }^{5}$ We use table 5 instead of table 6 to define the NLL spectrum for two reasons. First, since table 5 is a prescription commonly used, it provides a useful point of comparison, especially for the results in [26]. Second, the difference between the NLL and NLL' distributions is larger when using table 5, making the orders of accuracy more distinct. Using table 6, we find that the NLL distributions are more similar to NLL'.
} 
is no matching of the far tail to fixed-order perturbation theory, we only study the behavior of the distributions in the resummation region.

In figure 5, we plot the NLL resummed distributions for $\sigma_{n}\left(\tau_{a}\right)$ and $\sigma_{R}\left(\tau_{a}\right)$ for $a=$ $-1,0,1 / 4$, and $1 / 2$. In figure 6 we plot the NLL resummed distributions for the same values of $a$, comparing $\sigma_{R}\left(\tau_{a}\right)$ and $\sigma_{Q}\left(\tau_{a}\right)$. Figures 5 and 6 show a much better agreement between the dQCD distribution $\sigma_{Q}$ and the SCET distribution $\sigma_{R}$ (which is close to the derivative of the cumulant) than $\sigma_{Q}$ and the standard SCET distribution $\sigma_{n}$. In [26], angularity distributions in $\mathrm{dQCD}$ and SCET were compared using resummation formulae very similar to $\sigma_{n}$ and $\sigma_{Q}$. A discrepancy was observed which is very similar to the discrepancy seen here in figure 5 , and we see that it is ameliorated by including the additional resummed terms present in $\sigma_{R}$. The good agreement between $\sigma_{R}$ and $\sigma_{Q}$ in figure 6 provides strong evidence to support the analytic arguments that the dQCD and SCET resummations are in close correspondence. We note when $\tau_{a} \lesssim 0.01$, the central soft scale $\mu_{S}=Q \tau_{a} \lesssim 1 \mathrm{GeV}$ becomes nonperturbative and the predictions become unreliable without additional care.

The uncertainty bands on each distribution are determined by the envelope of scale variations, as described above. The relative size of uncertainty bands on $\sigma_{n}$ and $\sigma_{R}$ arise because of the relationship of $\sigma_{R}$ to the cumulant. In the large $\tau_{a}$ regime, the distribution $\sigma_{n}$ produces reasonable uncertainties. The cumulant has scale uncertainties whose $\tau_{a}$ dependence is very similar between different variations, meaning the derivative of the cumulant (which matches $\sigma_{R}$ closely) has very small scale uncertainties at large $\tau_{a}$. This is observed in figure 5 . We also note that at large $\tau_{a}, \sigma_{Q}$ has scale variation that is smaller than $\sigma_{n}$ but larger than $\sigma_{R}$, suggesting that more careful scale variations (with profiles) are needed when using $\sigma_{Q}$ in phenomenological applications. This is also evident in the fact that $\sigma_{Q}$ has very large uncertainties in the peak region. Finally, the increase in uncertainties with $a$ is due to the logarithmic structure of the resummation and the gradual breakdown of the resummation framework for both dQCD and SCET as $a \rightarrow 1$. Consistent uncertainties across $a$ values may be achieved with more careful scale variation and profile functions, and further study on how to obtain robust uncertainties for $\sigma_{R}$ and $\sigma_{Q}$ is needed.

In figure 7, we plot the NLL' resummed distributions for $\sigma_{n}\left(\tau_{a}\right)$ and $\sigma_{R}\left(\tau_{a}\right)$ for $a=$ $-1,0,1 / 4$, and $1 / 2$. In figure 8 we compare $\sigma_{R}\left(\tau_{a}\right)$ and $\sigma_{Q}\left(\tau_{a}\right)$ at NLL' accuracy at the same values of $a$. These figures can be contrasted with figures 5 and 6 .

The agreement between the central values of $\sigma_{R}$ and $\sigma_{Q}$ improves from NLL to NLL' in the region of $\tau_{a}$ past the peak. The moderate difference between them in the peak of the distribution observed in figure 8 arises from the different treatment in $\sigma_{R}$ and $\sigma_{Q}$ of the $\mathcal{O}\left(\alpha_{s}\right)$ nonlogarithmic singular terms that are included in the fixed-order functions at NLL'. We note this difference remains small compared to the uncertainties in the $\sigma_{Q}$ distribution.

The uncertainties in $\sigma_{n}, \sigma_{R}$, and $\sigma_{Q}$ at NLL' follow the same general pattern as the NLL case. An exception is that the uncertainties in the $\sigma_{n}$ distribution decrease noticeably in working to NLL'; this occurs because $\sigma_{n}$ at NLL contains the hard, jet, and soft functions only at tree level, while they are taken to $\mathcal{O}\left(\alpha_{s}\right)$ in the NLL' spectrum. In $\sigma_{R}$ and $\sigma_{Q}$, however, the NLL spectrum has contributions from the hard, jet, and soft functions beyond tree level. Had we used the prescription in table 6 to define the NLL spectrum for $\sigma_{n}$, we would find the uncertainties in that case are much closer to the NLL' spectrum in figure 7. 

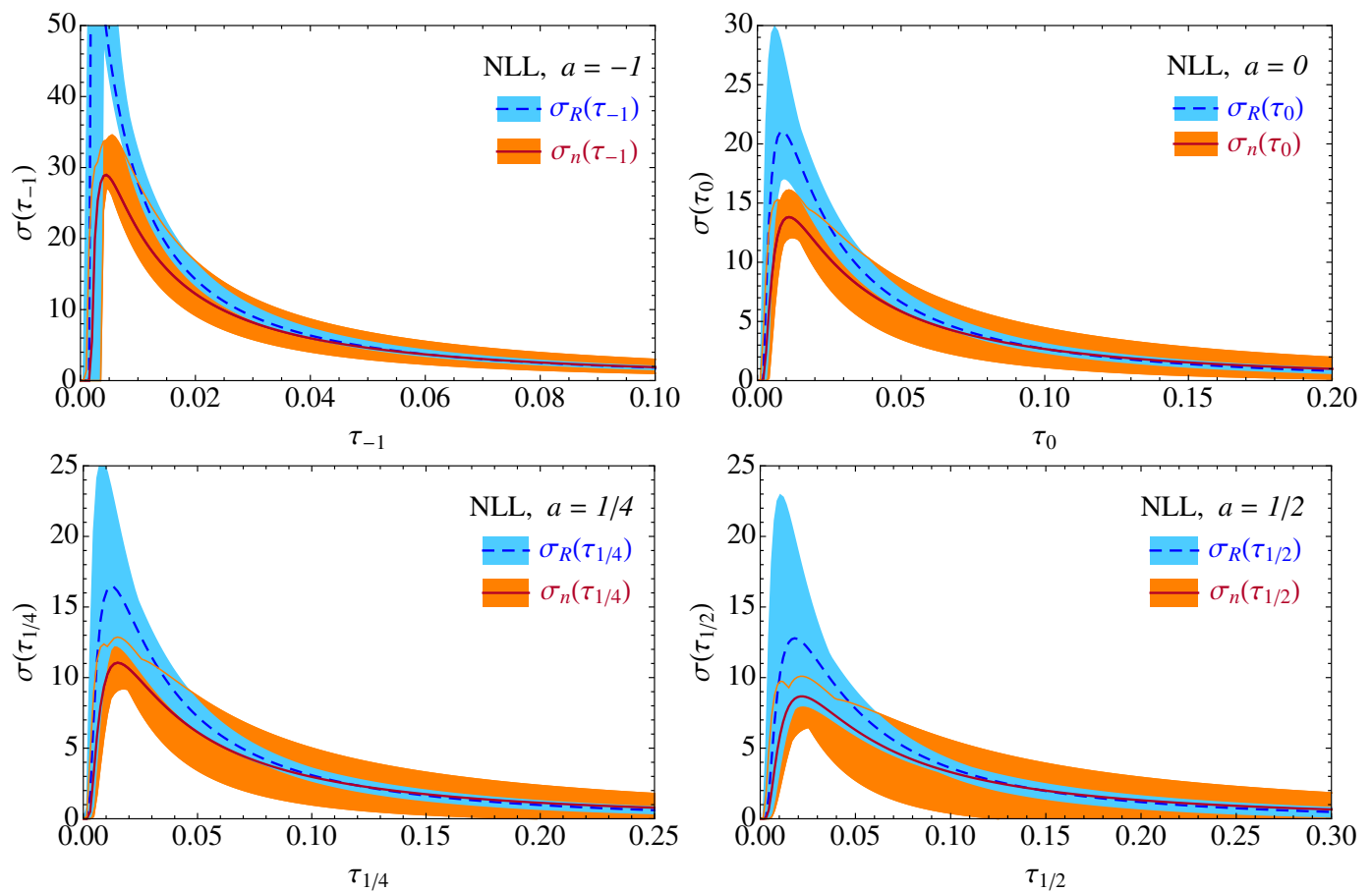

Figure 5. The NLL distributions for $\sigma_{n}\left(\tau_{a}\right)$ and $\sigma_{R}\left(\tau_{a}\right)$, for $a=-1,0,1 / 4$, and $1 / 2$ at $Q=$ $100 \mathrm{GeV}$. These compare the natural SCET resummation $\left(\sigma_{n}\right)$ with the resummed form that closely matches the derivative of the cumulant $\left(\sigma_{R}\right)$.

Further study of the relationship between the SCET and dQCD resummed angularity distributions is of interest to more deeply probe the numerical effect of the different approaches to resummation. As these approaches are formally consistent when working to the same order of accuracy, the relative agreement gives insight into the uncertainties that accompany each resummation scheme. Of particular interest would be a careful study that includes matching to fixed-order perturbation theory in the large $\tau_{a}$ tail region of the distribution and a systematic study of the profile scales and variations needed to obtain reliable uncertainties across the spectrum.

\section{Conclusions}

In this paper we have performed a detailed study of resummed $e^{+} e^{-}$event shape distributions in both SCET and dQCD, using angularities as a generic example. This study contains several parts, examining three different ways to express the cross sections: the cumulant $R\left(\tau_{a}\right)$, the spectrum $\sigma\left(\tau_{a}\right)$, and its Laplace transform $\widetilde{\sigma}(\nu)$.

In section 2 we reviewed standard logarithmic counting schemes. As the cross section in Laplace space directly exponentiates, it is simplest to define logarithmic accuracy by counting in the exponent of $\widetilde{\sigma}(\nu)$. We also discussed the original CTTW convention that determines logarithmic accuracy by counting in the exponent of the cumulant $R\left(\tau_{a}\right)$. While either definition of logarithmic accuracy is valid, and one can translate between the two, the advantage of defining accuracy in terms of the Laplace-transformed cross section is 

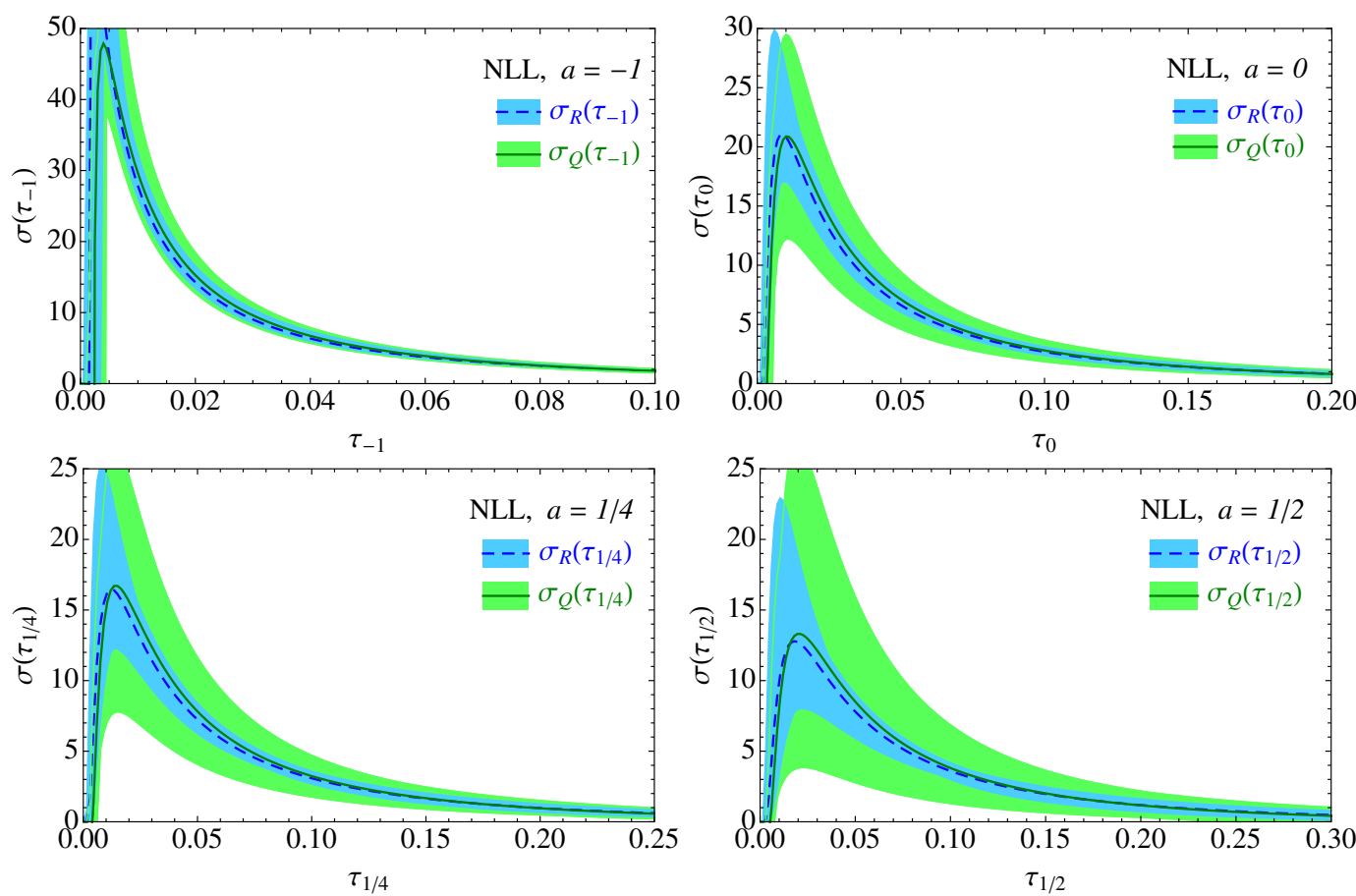

Figure 6. The NLL distributions for $\sigma_{Q}\left(\tau_{a}\right)$ and $\sigma_{R}\left(\tau_{a}\right)$, for $a=-1,0,1 / 4$, and $1 / 2$ at $Q=$ $100 \mathrm{GeV}$. These compare the dQCD-like resummation $\left(\sigma_{Q}\right)$ with the resummed form that closely matches the derivative of the cumulant $\left(\sigma_{R}\right)$. Note that these forms are in much better agreement than the comparison in figure 5 .

that a closed algebraic form for $\widetilde{\sigma}(\nu)$ is easily obtained. A major goal of section 4 is to define prescriptions for $R\left(\tau_{a}\right)$ and $\sigma\left(\tau_{a}\right)$ that are correct to a given order of accuracy when these cross sections are transformed to $\widetilde{\sigma}(\nu)$.

In section 3, we reviewed resummation techniques in SCET and dQCD and showed the equivalence between resummed forms, culminating in the equivalence relations eqs. (3.73), (3.78), and (3.84). This equivalence reveals alternative forms of the resummed cross section in SCET that more closely correspond with the resummed cross section in $\mathrm{dQCD}$, as well as a form of the $\mathrm{dQCD}$ cross section with dependence on arbitrary soft and collinear factorization scales, results which generalize both approaches to resummation.

In section 4 we discussed the precise prescriptions needed to obtain a given order of logarithmic accuracy. We explained how to compute the resummed $R\left(\tau_{a}\right)$ and distribution $\sigma\left(\tau_{a}\right)$ so that their accuracies match that of $\widetilde{\sigma}(\nu)$ after Laplace transformation. We also studied a way of defining the resummed spectrum $\sigma\left(\tau_{a}\right)$ in terms of the resummed cumulant $R\left(\tau_{a}\right)$ [or equivalently the Laplace-transformed cross section $\widetilde{\sigma}(\nu)$ ]. This leads to a novel way, eq. (4.67), of writing the resummed spectrum that matches onto the derivative of the cumulant.

A compact summary of the dQCD and SCET resummed forms for the cross section, its Laplace transform, and the cumulant is given in section 5 .

Finally, in section 6 we have performed a short numerical study of the angularity distributions. We have shown that numerical discrepancies between the dQCD forms (given 



Figure 7. The NLL' distributions for $\sigma_{n}\left(\tau_{a}\right)$ and $\sigma_{R}\left(\tau_{a}\right)$, for $a=-1,0,1 / 4$, and $1 / 2$ at $Q=$ $100 \mathrm{GeV}$. These compare the natural SCET resummation $\left(\sigma_{n}\right)$ with the resummed form that closely matches the derivative of the cumulant $\left(\sigma_{R}\right)$.

in $[12,13]$ ) and the SCET forms (given in $[26]$ ), originally observed in the latter study, are resolved by using versions of the resummed cross sections that arise from the equivalence of the dQCD and SCET forms. This gives confidence that the novel resummed forms derived in this paper may be applied in phenomenological studies. For example, we are now in a position to perform a robust comparison to LEP data in [87]. Further work is warranted to study the resummed forms introduced here at higher resummed orders and to determine techniques to achieve robust uncertainty estimates. It would also be informative to extend our study to resummation of angularities with $a \geq 1$ [54-58] or to the recently introduced "recoil-free" observables [59].

Although the comparisons and lessons in this paper are formulated in terms of event shape distributions in $e^{+} e^{-}$collisions, the observations about how to compute different ways of writing a cross section to consistent accuracy are applicable generally to any cross section computed in dQCD. In the paper [34] which appeared recently, similar comparisons are performed for threshold resummation in hadron collisions.

\section{Acknowledgments}

We thank the organizers of the 2010 "Joint Theoretical-Experimental Workshop on Jets and Jet Substructure at the LHC" at the University of Washington (supported by DOE Contract DE-FG02-96ER40956) where this project was germinated, and the organizers of the 2011 INT Program on "Frontiers of QCD", the 2012 Boston Jet Physics Workshop, and the 2013 ESI Program on "Jets and Quantum Fields for LHC and Future Colliders" 

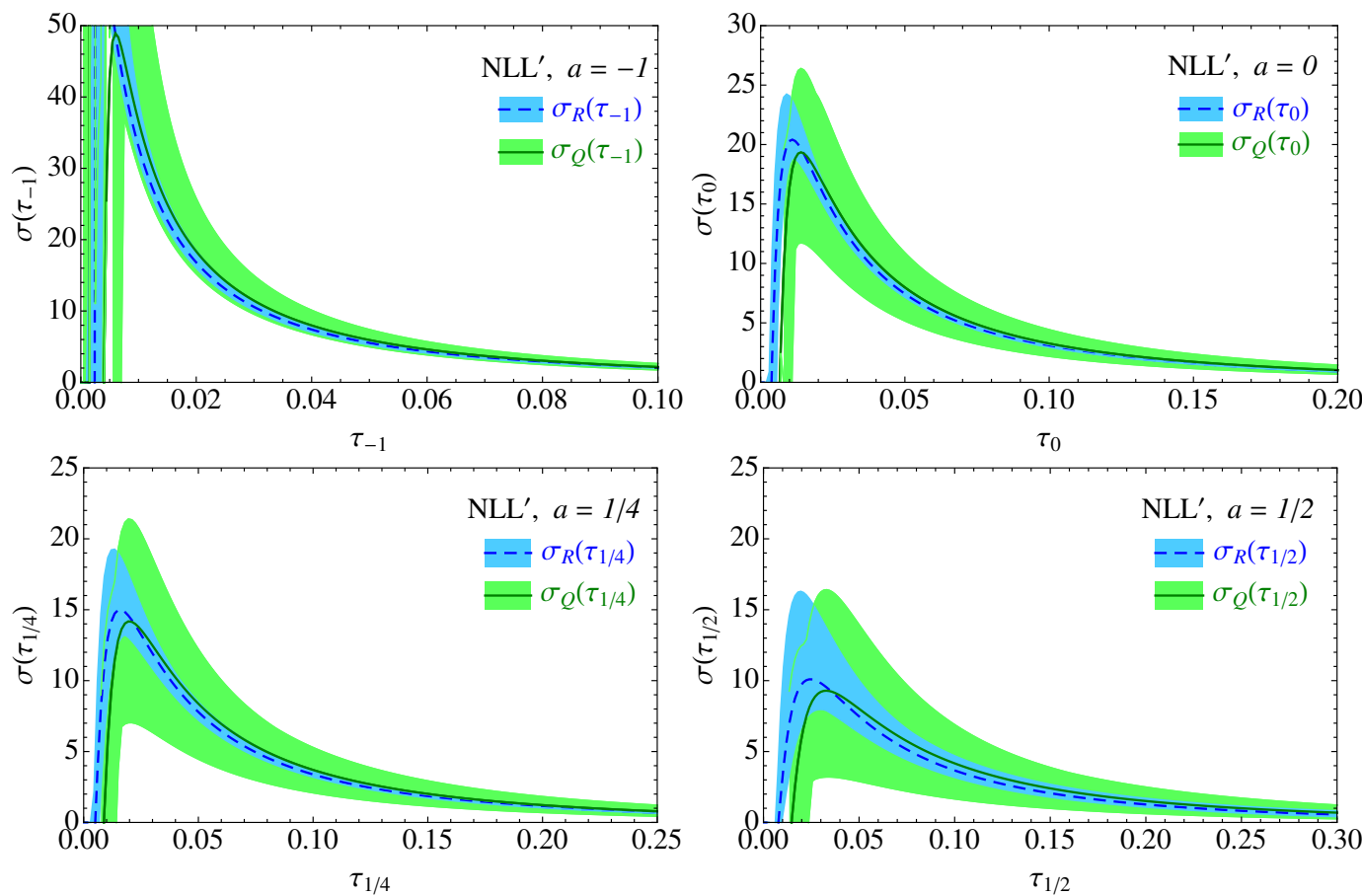

Figure 8. The NLL' distributions for $\sigma_{Q}\left(\tau_{a}\right)$ and $\sigma_{R}\left(\tau_{a}\right)$, for $a=-1,0,1 / 4$, and $1 / 2$ at $Q=$ $100 \mathrm{GeV}$. These compare the dQCD-like resummation $\left(\sigma_{Q}\right)$ with the resummed form that closely matches the derivative of the cumulant $\left(\sigma_{R}\right)$. Note that these forms are in better agreement than the comparison in figure 7 .

in Vienna, Austria, where it progressed. We thank S. Rappoccio for asking the question that instigated this project. GS thanks M. Zeng, and CL and JRW thank I. Stewart and F. Tackmann, for many useful discussions. We also thank the CNYITP at Stony Brook (CL and JRW) and Los Alamos National Laboratory (JRW) for hospitality during portions of this work. The work of LGA was partially supported by the P2IO Labex. The work of SDE was supported by DOE Contract DE-FG02-96ER40956. The work of CL is supported by DOE Contract DE-AC52-06NA25396 and by the LDRD office at Los Alamos. The work of GS, IS, and LGA was supported by the National Science Foundation, grants PHY-0653342, PHY-0969739 and PHY-1316617. The work of IS was supported by DOE Contract DE-FG02-93ER40762. The work of JRW was supported by DOE Contract DE-AC02-05CH11231, and the LHC Theory Initiative, US National Science Foundation grant NSF-PHY-0705682.

\section{A Plus distributions}

In this appendix we collect definitions and properties of plus distributions used in the main text.

In general for a function $q$, the plus distribution is defined by (see, e.g., [76])

$$
\begin{aligned}
{[q(x)]_{+} } & =\lim _{\epsilon \rightarrow 0} \frac{d}{d x}[\theta(x-\epsilon) Q(x)] \\
& =\lim _{\epsilon \rightarrow 0}[\theta(x-\epsilon) q(x)+\delta(x-\epsilon) Q(x)],
\end{aligned}
$$


where

$$
Q(x)=\int_{1}^{x} d x^{\prime} q\left(x^{\prime}\right) .
$$

With the choice of lower limit in eq. (A.2), the definition in eq. (A.1) satisfies $\int_{0}^{1} d x[q(x)]_{+}=$ 0 . The result of integrating a plus distribution against a suitable test function $f(x)$ is

$$
\int_{-\infty}^{x_{\max }} d x[\theta(x) q(x)]_{+} f(x)=\int_{0}^{x_{\max }} d x q(x)[f(x)-f(0)]+f(0) Q\left(x_{\max }\right) .
$$

We define two special plus distributions which commonly appear,

$$
\mathcal{L}_{n}(x) \equiv\left[\frac{\theta(x) \ln ^{n} x}{x}\right]_{+},(n \geq 0), \quad \mathcal{L}^{a}(x) \equiv\left[\frac{\theta(x)}{x^{1-a}}\right]_{+} .
$$

For the case $n=-1$, we define

$$
\mathcal{L}_{-1}(x) \equiv \delta(x) .
$$

The plus function $\mathcal{L}_{n}$ obeys the rescaling relation,

$$
\lambda \mathcal{L}_{n}(\lambda x)=\sum_{k=0}^{n}\left(\begin{array}{l}
n \\
k
\end{array}\right) \ln ^{k} \lambda \mathcal{L}_{n-k}(x)+\frac{\ln ^{n+1} \lambda}{n+1} \delta(x)
$$

\section{B Laplace transforms}

In this appendix we collect results for the Laplace transforms and inverse Laplace transforms $\left(\mathscr{L}^{-1}\right)$ between the $\operatorname{logs}$

$$
L \equiv \ln \frac{1}{\tau}, \quad \tilde{L} \equiv \ln \left(\nu e^{\gamma_{E}}\right)
$$

The Laplace transforms, defined by

$$
\widetilde{F}(\nu) \equiv \mathscr{L}\{F\}(\nu)=\int_{0}^{\infty} d \tau e^{-\nu \tau} F(\tau)
$$

are given by

$$
\begin{aligned}
\mathscr{L}\{1\} & =\frac{1}{\nu} \\
\mathscr{L}\{L\} & =\frac{1}{\nu} \tilde{L} \\
\mathscr{L}\left\{L^{2}\right\} & =\frac{1}{\nu}\left\{\tilde{L}^{2}+\frac{\pi^{2}}{6}\right\} \\
\mathscr{L}\left\{L^{3}\right\} & =\frac{1}{\nu}\left\{\tilde{L}^{3}+\frac{\pi^{2}}{2} \tilde{L}+2 \zeta_{3}\right\} \\
\mathscr{L}\left\{L^{4}\right\} & =\frac{1}{\nu}\left\{\tilde{L}^{4}+\pi^{2} \tilde{L}^{2}+8 \zeta_{3} \tilde{L}+\frac{3 \pi^{4}}{20}\right\} \\
\mathscr{L}\left\{L^{5}\right\} & =\frac{1}{\nu}\left\{\tilde{L}^{5}+\frac{5 \pi^{2}}{3} \tilde{L}^{3}+20 \zeta_{3} \tilde{L}^{2}+\frac{3 \pi^{4}}{4} \tilde{L}+\frac{10 \pi^{2}}{3} \zeta_{3}+24 \zeta_{5}\right\} \\
\mathscr{L}\left\{L^{6}\right\} & =\frac{1}{\nu}\left\{\tilde{L}^{6}+\frac{5 \pi^{2}}{2} \tilde{L}^{4}+40 \zeta_{3} \tilde{L}^{3}+\frac{9 \pi^{4}}{4} \tilde{L}^{2}+\left(20 \pi^{2} \zeta_{3}+144 \zeta_{5}\right) \tilde{L}+40 \zeta_{3}^{2}+\frac{61 \pi^{6}}{168}\right\}
\end{aligned}
$$


and so on. The inverse Laplace transforms, defined by

$$
\mathscr{L}^{-1}\{\widetilde{F}\}(\tau)=\int_{\gamma-i \infty}^{\gamma+i \infty} \frac{d \nu}{2 \pi i} e^{\nu \tau} \widetilde{F}(\nu),
$$

where $\gamma$ lies to the right of all the poles of $\widetilde{F}$ in the complex plane, are given by

$$
\begin{aligned}
\mathscr{L}^{-1}\left\{\frac{1}{\nu}\right\} & =1 \\
\mathscr{L}^{-1}\left\{\frac{1}{\nu} \tilde{L}\right\} & =L \\
\mathscr{L}^{-1}\left\{\frac{1}{\nu} \tilde{L}^{2}\right\} & =L^{2}-\frac{\pi^{2}}{6} \\
\mathscr{L}^{-1}\left\{\frac{1}{\nu} \tilde{L}^{3}\right\} & =L^{3}-\frac{\pi^{2}}{2} L-2 \zeta_{3} \\
\mathscr{L}^{-1}\left\{\frac{1}{\nu} \tilde{L}^{4}\right\} & =L^{4}-\pi^{2} L^{2}-8 \zeta_{3} L+\frac{\pi^{4}}{60} \\
\mathscr{L}^{-1}\left\{\frac{1}{\nu} \tilde{L}^{5}\right\} & =L^{5}-\frac{5 \pi^{2}}{3} L^{3}-20 \zeta_{3} L^{2}+\frac{\pi^{4}}{12} L+\frac{10 \pi^{2}}{3} \zeta_{3}-24 \zeta_{5} \\
\mathscr{L}^{-1}\left\{\frac{1}{\nu} \tilde{L}^{6}\right\} & =L^{6}-\frac{5 \pi^{2}}{2} L^{4}-40 \zeta_{3} L^{3}+\frac{\pi^{4}}{4} L^{2}+\left(20 \pi^{2} \zeta_{3}-144 \zeta_{5}\right) L+40 \zeta_{3}^{2}-\frac{5 \pi^{6}}{168},
\end{aligned}
$$

and so on. The results explicitly tabulated in eqs. (B.3) and (B.5) are needed to transform $\operatorname{logs}$ in the fixed-order expansions of event shape distributions in QCD up to $\mathcal{O}\left(\alpha_{s}^{3}\right)$.

\section{Anomalous dimensions}

The coefficients of the beta function up to three-loop order in $\overline{\mathrm{MS}}$ are given by $[88,89]$

$$
\begin{aligned}
& \beta_{0}=\frac{11}{3} C_{A}-\frac{4}{3} T_{F} n_{f}, \\
& \beta_{1}=\frac{34}{3} C_{A}^{2}-\left(\frac{20}{3} C_{A}+4 C_{F}\right) T_{F} n_{f}, \\
& \beta_{2}=\frac{2857}{54} C_{A}^{3}+\left(C_{F}^{2}-\frac{205}{18} C_{F} C_{A}-\frac{1415}{54} C_{A}^{2}\right) 2 T_{F} n_{f}+\left(\frac{11}{9} C_{F}+\frac{79}{54} C_{A}\right) 4 T_{F}^{2} n_{f}^{2},
\end{aligned}
$$

and the cusp anomalous dimension coefficients by [72, 73]:

$$
\begin{aligned}
& \Gamma_{0}^{q}=4 C_{F} \\
& \Gamma_{1}^{q}=4 C_{F} {\left[\left(\frac{67}{9}-\frac{\pi^{2}}{3}\right) C_{A}-\frac{20}{9} T_{F} n_{f}\right] } \\
& \Gamma_{2}^{q}=4 C_{F} {\left[\left(\frac{245}{6}-\frac{134 \pi^{2}}{27}+\frac{11 \pi^{4}}{45}+\frac{22 \zeta_{3}}{3}\right) C_{A}^{2}+\left(-\frac{418}{27}+\frac{40 \pi^{2}}{27}-\frac{56 \zeta_{3}}{3}\right) C_{A} T_{F} n_{f}\right.} \\
&\left.+\left(-\frac{55}{3}+16 \zeta_{3}\right) C_{F} T_{F} n_{f}-\frac{16}{27} T_{F}^{2} n_{f}^{2}\right]
\end{aligned}
$$


The $\overline{\mathrm{MS}}$ non-cusp anomalous dimension $\gamma_{H}=2 \gamma_{C}$ for the hard function $H$ can be obtained $[29,66]$ from the IR divergences of the on-shell massless quark form factor $C\left(q^{2}, \mu\right)$ which are known to three loops [65],

$$
\begin{aligned}
\gamma_{H}^{0}=-12 C_{F} & \\
\gamma_{H}^{1}=-2 C_{F} & {\left[\left(\frac{82}{9}-52 \zeta_{3}\right) C_{A}+\left(3-4 \pi^{2}+48 \zeta_{3}\right) C_{F}+\left(\frac{65}{9}+\pi^{2}\right) \beta_{0}\right], } \\
\gamma_{H}^{2}=-4 C_{F} & {\left[\left(\frac{66167}{324}-\frac{686 \pi^{2}}{81}-\frac{302 \pi^{4}}{135}-\frac{782 \zeta_{3}}{9}+\frac{44 \pi^{2} \zeta_{3}}{9}+136 \zeta_{5}\right) C_{A}^{2}\right.} \\
+ & \left(\frac{151}{4}-\frac{205 \pi^{2}}{9}-\frac{247 \pi^{4}}{135}+\frac{844 \zeta_{3}}{3}+\frac{8 \pi^{2} \zeta_{3}}{3}+120 \zeta_{5}\right) C_{F} C_{A} \\
+ & \left(\frac{29}{2}+3 \pi^{2}+\frac{8 \pi^{4}}{5}+68 \zeta_{3}-\frac{16 \pi^{2} \zeta_{3}}{3}-240 \zeta_{5}\right) C_{F}^{2} \\
+ & \left(-\frac{10781}{108}+\frac{446 \pi^{2}}{81}+\frac{449 \pi^{4}}{270}-\frac{1166 \zeta_{3}}{9}\right) C_{A} \beta_{0} \\
+ & \left.\left(\frac{2953}{108}-\frac{13 \pi^{2}}{18}-\frac{7 \pi^{4}}{27}+\frac{128 \zeta_{3}}{9}\right) \beta_{1}+\left(-\frac{2417}{324}+\frac{5 \pi^{2}}{6}+\frac{2 \zeta_{3}}{3}\right) \beta_{0}^{2}\right] .
\end{aligned}
$$

The non-cusp three-loop anomalous dimension for the $a=0$ quark jet function is given by [29],

$$
\begin{aligned}
\gamma_{J}^{0}=6 C_{F} & \\
\gamma_{J}^{1}=C_{F} & {\left[\left(\frac{146}{9}-80 \zeta_{3}\right) C_{A}+\left(3-4 \pi^{2}+48 \zeta_{3}\right) C_{F}+\left(\frac{121}{9}+\frac{2 \pi^{2}}{3}\right) \beta_{0}\right] } \\
\gamma_{J}^{2}=2 C_{F} & {\left[\left(\frac{52019}{162}-\frac{841 \pi^{2}}{81}-\frac{82 \pi^{4}}{27}-\frac{2056 \zeta_{3}}{9}+\frac{88 \pi^{2} \zeta_{3}}{9}+232 \zeta_{5}\right) C_{A}^{2}\right.} \\
+ & \left(\frac{151}{4}-\frac{205 \pi^{2}}{9}-\frac{247 \pi^{4}}{135}+\frac{844 \zeta_{3}}{3}+\frac{8 \pi^{2} \zeta_{3}}{3}+120 \zeta_{5}\right) C_{A} C_{F} \\
+ & \left(\frac{29}{2}+3 \pi^{2}+\frac{8 \pi^{4}}{5}+68 \zeta_{3}-\frac{16 \pi^{2} \zeta_{3}}{3}-240 \zeta_{5}\right) C_{F}^{2} \\
+ & \left(-\frac{7739}{54}+\frac{325}{81} \pi^{2}+\frac{617 \pi^{4}}{270}-\frac{1276 \zeta_{3}}{9}\right) C_{A} \beta_{0} \\
+ & \left.\left(-\frac{3457}{324}+\frac{5 \pi^{2}}{9}+\frac{16 \zeta_{3}}{3}\right) \beta_{0}^{2}+\left(\frac{1166}{27}-\frac{8 \pi^{2}}{9}-\frac{41 \pi^{4}}{135}+\frac{52 \zeta_{3}}{9}\right) \beta_{1}\right] .
\end{aligned}
$$

The anomalous dimension for the soft function is obtained from $\gamma_{S}=-\gamma_{H}-2 \gamma_{J}$.

Open Access. This article is distributed under the terms of the Creative Commons Attribution License (CC-BY 4.0), which permits any use, distribution and reproduction in any medium, provided the original author(s) and source are credited.

\section{References}

[1] D.J. Gross and F. Wilczek, Ultraviolet behavior of nonabelian gauge theories, Phys. Rev. Lett. 30 (1973) 1343 [INSPIRE]. 
[2] H.D. Politzer, Reliable perturbative results for strong interactions?, Phys. Rev. Lett. 30 (1973) 1346 [inSPIRE].

[3] G.F. Sterman and S. Weinberg, Jets from quantum chromodynamics, Phys. Rev. Lett. 39 (1977) 1436 [InSPIRE].

[4] M. Dasgupta and G.P. Salam, Event shapes in $e^{+} e^{-}$annihilation and deep inelastic scattering, J. Phys. G 30 (2004) R143 [hep-ph/0312283] [INSPIRE].

[5] A. Hornig, C. Lee, I.W. Stewart, J.R. Walsh and S. Zuberi, Non-global structure of the $O\left(\alpha_{s}^{2}\right)$ dijet soft function, JHEP 08 (2011) 054 [arXiv:1105.4628] [INSPIRE].

[6] M. Dasgupta and G. Salam, Resummation of nonglobal QCD observables, Phys. Lett. B 512 (2001) 323 [hep-ph/0104277] [INSPIRE].

[7] E. Farhi, A QCD test for jets, Phys. Rev. Lett. 39 (1977) 1587 [inSPIRE].

[8] V. Mateu, I.W. Stewart and J. Thaler, Power corrections to event shapes with mass-dependent operators, Phys. Rev. D 87 (2013) 014025 [arXiv:1209.3781] [InSPIRE].

[9] G. Salam and D. Wicke, Hadron masses and power corrections to event shapes, JHEP 05 (2001) 061 [hep-ph/0102343] [INSPIRE].

[10] G.F. Sterman, Mass divergences in annihilation processes. 2. Cancellation of divergences in cut vacuum polarization diagrams, Phys. Rev. D 17 (1978) 2789 [InSPIRE].

[11] G. Sterman, Infrared divergences in perturbative $Q C D$, in the proceedings of the Perturbative quantum chromodynamics, March 25-28, Thallassee, U.S.A. (1981).

[12] C.F. Berger, T. Kucs and G.F. Sterman, Event shape/energy flow correlations, Phys. Rev. D 68 (2003) 014012 [hep-ph/0303051] [INSPIRE].

[13] C.F. Berger and G.F. Sterman, Scaling rule for nonperturbative radiation in a class of event shapes, JHEP 09 (2003) 058 [hep-ph/0307394] [INSPIRE].

[14] C.F. Berger and L. Magnea, Scaling of power corrections for angularities from dressed gluon exponentiation, Phys. Rev. D 70 (2004) 094010 [hep-ph/0407024] [INSPIRE].

[15] S. Catani, L. Trentadue, G. Turnock and B. Webber, Resummation of large logarithms in $e^{+} e^{-}$event shape distributions, Nucl. Phys. B 407 (1993) 3 [InSPIRE].

[16] H. Contopanagos, E. Laenen and G.F. Sterman, Sudakov factorization and resummation, Nucl. Phys. B 484 (1997) 303 [hep-ph/9604313] [INSPIRE].

[17] C.W. Bauer, S. Fleming, D. Pirjol, I.Z. Rothstein and I.W. Stewart, Hard scattering factorization from effective field theory, Phys. Rev. D 66 (2002) 014017 [hep-ph/0202088] [INSPIRE].

[18] C.W. Bauer, S. Fleming and M.E. Luke, Summing Sudakov logarithms in $B \rightarrow X(s \gamma)$ in effective field theory, Phys. Rev. D 63 (2000) 014006 [hep-ph/0005275] [INSPIRE].

[19] C.W. Bauer, S. Fleming, D. Pirjol and I.W. Stewart, An effective field theory for collinear and soft gluons: heavy to light decays, Phys. Rev. D 63 (2001) 114020 [hep-ph/0011336] [INSPIRE].

[20] C.W. Bauer and I.W. Stewart, Invariant operators in collinear effective theory, Phys. Lett. B 516 (2001) 134 [hep-ph/0107001] [INSPIRE].

[21] C.W. Bauer, D. Pirjol and I.W. Stewart, Soft collinear factorization in effective field theory, Phys. Rev. D 65 (2002) 054022 [hep-ph/0109045] [INSPIRE]. 
[22] C.W. Bauer, C. Lee, A.V. Manohar and M.B. Wise, Enhanced nonperturbative effects in Z decays to hadrons, Phys. Rev. D 70 (2004) 034014 [hep-ph/0309278] [INSPIRE].

[23] C.W. Bauer, A.V. Manohar and M.B. Wise, Enhanced nonperturbative effects in jet distributions, Phys. Rev. Lett. 91 (2003) 122001 [hep-ph/0212255] [INSPIRE].

[24] C.W. Bauer, S.P. Fleming, C. Lee and G.F. Sterman, Factorization of $e^{+} e^{-}$event shape distributions with hadronic final states in soft collinear effective theory, Phys. Rev. D 78 (2008) 034027 [arXiv:0801.4569] [INSPIRE].

[25] A.V. Manohar, Effective field theories, hep-ph/9606222 [INSPIRE].

[26] A. Hornig, C. Lee and G. Ovanesyan, Effective predictions of event shapes: factorized, resummed and gapped angularity distributions, JHEP 05 (2009) 122 [arXiv:0901.3780] [INSPIRE].

[27] A. Hornig, C. Lee and G. Ovanesyan, Infrared safety in factorized hard scattering cross-sections, Phys. Lett. B 677 (2009) 272 [arXiv:0901.1897] [INSPIRE].

[28] A. Banfi, G.P. Salam and G. Zanderighi, Principles of general final-state resummation and automated implementation, JHEP 03 (2005) 073 [hep-ph/0407286] [INSPIRE].

[29] T. Becher, M. Neubert and B.D. Pecjak, Factorization and momentum-space resummation in deep-inelastic scattering, JHEP 01 (2007) 076 [hep-ph/0607228] [INSPIRE].

[30] T. Becher and M. Neubert, Threshold resummation in momentum space from effective field theory, Phys. Rev. Lett. 97 (2006) 082001 [hep-ph/0605050] [INSPIRE].

[31] A.V. Manohar, Deep inelastic scattering as $x \rightarrow 1$ using soft collinear effective theory, Phys. Rev. D 68 (2003) 114019 [hep-ph/0309176] [INSPIRE].

[32] M. Bonvini, S. Forte, M. Ghezzi and G. Ridolfi, Threshold resummation in SCET vs. perturbative QCD: an analytic comparison, Nucl. Phys. B 861 (2012) 337 [arXiv:1201.6364] [INSPIRE].

[33] M. Bonvini, S. Forte, M. Ghezzi and G. Ridolfi, The scale of soft resummation in SCET vs perturbative QCD, Nucl. Phys. Proc. Suppl. 241-242 (2013) 121 [arXiv:1301.4502] [INSPIRE].

[34] G. Sterman and M. Zeng, Quantifying comparisons of threshold resummations, arXiv:1312.5397 [INSPIRE].

[35] G.P. Korchemsky and G.F. Sterman, Power corrections to event shapes and factorization, Nucl. Phys. B 555 (1999) 335 [hep-ph/9902341] [INSPIRE].

[36] G. Korchemsky and S. Tafat, On power corrections to the event shape distributions in QCD, JHEP 10 (2000) 010 [hep-ph/0007005] [INSPIRE].

[37] A.H. Hoang and I.W. Stewart, Designing gapped soft functions for jet production, Phys. Lett. B 660 (2008) 483 [arXiv:0709.3519] [INSPIRE].

[38] R. Abbate, M. Fickinger, A.H. Hoang, V. Mateu and I.W. Stewart, Thrust at $N^{3} L L$ with power corrections and a precision global fit for $\alpha_{s}\left(m_{Z}\right)$, Phys. Rev. D 83 (2011) 074021 [arXiv: 1006.3080] [INSPIRE].

[39] J.-y. Chiu, F. Golf, R. Kelley and A.V. Manohar, Electroweak corrections in high energy processes using effective field theory, Phys. Rev. D 77 (2008) 053004 [arXiv:0712.0396] [INSPIRE]. 
[40] S.D. Ellis, A. Hornig, C. Lee, C.K. Vermilion and J.R. Walsh, Consistent factorization of jet observables in exclusive multijet cross-sections, Phys. Lett. B 689 (2010) 82 [arXiv: 0912.0262] [INSPIRE].

[41] S.D. Ellis, C.K. Vermilion, J.R. Walsh, A. Hornig and C. Lee, Jet shapes and jet algorithms in SCET, JHEP 11 (2010) 101 [arXiv:1001.0014] [INSPIRE].

[42] I.W. Stewart, F.J. Tackmann and W.J. Waalewijn, N-jettiness: an inclusive event shape to veto jets, Phys. Rev. Lett. 105 (2010) 092002 [arXiv: 1004.2489] [INSPIRE].

[43] J. Thaler and K. Van Tilburg, Identifying boosted objects with $\mathrm{N}$-subjettiness, JHEP 03 (2011) 015 [arXiv: 1011.2268] [INSPIRE].

[44] C.W. Bauer, F.J. Tackmann, J.R. Walsh and S. Zuberi, Factorization and resummation for dijet invariant mass spectra, Phys. Rev. D 85 (2012) 074006 [arXiv:1106.6047] [inSPIRE].

[45] A. Altheimer et al., Jet Substructure at the Tevatron and LHC: new results, new tools, new benchmarks, J. Phys. G 39 (2012) 063001 [arXiv:1201.0008] [INSPIRE].

[46] A. Abdesselam et al., Boosted objects: a probe of beyond the standard model physics, Eur. Phys. J. C 71 (2011) 1661 [arXiv:1012.5412] [INSPIRE].

[47] A. Altheimer et al., Boosted objects and jet substructure at the LHC, arXiv:1311.2708 [INSPIRE].

[48] C. Lee and G.F. Sterman, Momentum flow correlations from event shapes: factorized soft gluons and soft-collinear effective theory, Phys. Rev. D 75 (2007) 014022 [hep-ph/0611061] [INSPIRE].

[49] L. Clavelli, Jet invariant mass in quantum chromodynamics, Phys. Lett. B 85 (1979) 111 [INSPIRE].

[50] L. Clavelli and D. Wyler, Kinematical bounds on jet variables and the heavy jet mass distribution, Phys. Lett. B 103 (1981) 383 [INSPIRE].

[51] T. Chandramohan and L. Clavelli, Consequences of second order QCD for jet structure in $e^{+} e^{-}$annihilation, Nucl. Phys. B 184 (1981) 365 [INSPIRE].

[52] S. Catani, G. Turnock and B. Webber, Jet broadening measures in $e^{+} e^{-}$annihilation, Phys. Lett. B 295 (1992) 269 [INSPIRE].

[53] R.K. Ellis, D.A. Ross and A.E. Terrano, The perturbative calculation of jet structure in $e^{+} e^{-}$ annihilation, Nucl. Phys. B 178 (1981) 421 [INSPIRE].

[54] T. Becher, G. Bell and M. Neubert, Factorization and resummation for jet broadening, Phys. Lett. B 704 (2011) 276 [arXiv:1104.4108] [INSPIRE].

[55] J.-y. Chiu, A. Jain, D. Neill and I.Z. Rothstein, The rapidity renormalization group, Phys. Rev. Lett. 108 (2012) 151601 [arXiv:1104.0881] [INSPIRE].

[56] J.-Y. Chiu, A. Jain, D. Neill and I.Z. Rothstein, A formalism for the systematic treatment of rapidity logarithms in quantum field theory, JHEP 05 (2012) 084 [arXiv:1202.0814] [INSPIRE].

[57] T. Becher and G. Bell, NNLL resummation for jet broadening, JHEP 11 (2012) 126 [arXiv: 1210.0580] [INSPIRE].

[58] Y.L. Dokshitzer, A. Lucenti, G. Marchesini and G. Salam, On the QCD analysis of jet broadening, JHEP 01 (1998) 011 [hep-ph/9801324] [INSPIRE]. 
[59] A.J. Larkoski, D. Neill and J. Thaler, Jet shapes with the broadening axis, JHEP 04 (2014) 017 [arXiv: 1401.2158] [INSPIRE].

[60] S.M. Freedman, Subleading corrections to thrust using effective field theory, arXiv:1303.1558 [INSPIRE].

[61] C. Lee and G.F. Sterman, Universality of nonperturbative effects in event shapes, eConf $\mathbf{C}$ 0601121 (2006) A001 [hep-ph/0603066] [inSPIRE].

[62] A.V. Manohar and I.W. Stewart, The zero-bin and mode factorization in quantum field theory, Phys. Rev. D 76 (2007) 074002 [hep-ph/0605001] [INSPIRE].

[63] A. Idilbi and T. Mehen, On the equivalence of soft and zero-bin subtractions, Phys. Rev. D 75 (2007) 114017 [hep-ph/0702022] [INSPIRE].

[64] A. Idilbi and T. Mehen, Demonstration of the equivalence of soft and zero-bin subtractions, Phys. Rev. D 76 (2007) 094015 [arXiv:0707.1101] [INSPIRE].

[65] S. Moch, J. Vermaseren and A. Vogt, The quark form-factor at higher orders, JHEP 08 (2005) 049 [hep-ph/0507039] [inSPIRE].

[66] A. Idilbi, X.-d. Ji and F. Yuan, Resummation of threshold logarithms in effective field theory for DIS, Drell-Yan and Higgs production, Nucl. Phys. B 753 (2006) 42 [hep-ph/0605068] [INSPIRE].

[67] C.W. Bauer and A.V. Manohar, Shape function effects in $B \rightarrow X_{s} \gamma$ and $B \rightarrow X_{u} l \overline{n u}$ decays, Phys. Rev. D 70 (2004) 034024 [hep-ph/0312109] [InSPIRE].

[68] T. Becher and M. Neubert, Toward a NNLO calculation of the $\bar{B} \rightarrow X_{s} \gamma$ decay rate with a cut on photon energy. II. Two-loop result for the jet function, Phys. Lett. B 637 (2006) 251 [hep-ph/0603140] [INSPIRE].

[69] R. Kelley, M.D. Schwartz, R.M. Schabinger and H.X. Zhu, The two-loop hemisphere soft function, Phys. Rev. D 84 (2011) 045022 [arXiv:1105.3676] [INSPIRE].

[70] P.F. Monni, T. Gehrmann and G. Luisoni, Two-loop soft corrections and resummation of the thrust distribution in the dijet region, JHEP 08 (2011) 010 [arXiv:1105.4560] [INSPIRE].

[71] T. Becher and M.D. Schwartz, A precise determination of $\alpha_{s}$ from LEP thrust data using effective field theory, JHEP 07 (2008) 034 [arXiv: 0803.0342] [INSPIRE].

[72] G. Korchemsky and A. Radyushkin, Renormalization of the Wilson loops beyond the leading order, Nucl. Phys. B 283 (1987) 342 [INSPIRE].

[73] S. Moch, J. Vermaseren and A. Vogt, The three loop splitting functions in QCD: the nonsinglet case, Nucl. Phys. B 688 (2004) 101 [hep-ph/0403192] [INSPIRE].

[74] G. Korchemsky and G. Marchesini, Resummation of large infrared corrections using Wilson loops, Phys. Lett. B 313 (1993) 433 [InSPIRE].

[75] C. Balzereit, T. Mannel and W. Kilian, Evolution of the light cone distribution function for a heavy quark, Phys. Rev. D 58 (1998) 114029 [hep-ph/9805297] [INSPIRE].

[76] Z. Ligeti, I.W. Stewart and F.J. Tackmann, Treating the $b$ quark distribution function with reliable uncertainties, Phys. Rev. D 78 (2008) 114014 [arXiv:0807.1926] [INSPIRE].

[77] H. Contopanagos and G.F. Sterman, Normalization of the Drell-Yan cross-section in QCD, Nucl. Phys. B 400 (1993) 211 [nSPIRE]. 
[78] D. Kang, C. Lee and I.W. Stewart, Using 1-jettiness to measure 2 jets in DIS 3 ways, Phys. Rev. D 88 (2013) 054004 [arXiv: 1303.6952] [INSPIRE].

[79] C.F. Berger, C. Marcantonini, I.W. Stewart, F.J. Tackmann and W.J. Waalewijn, Higgs production with a central jet veto at NNLL+NNLO, JHEP 04 (2011) 092 [arXiv: 1012.4480] [INSPIRE].

[80] J.C. Collins and D.E. Soper, Back-to-back jets in QCD, Nucl. Phys. B 193 (1981) 381 [Erratum ibid. B 213 (1983) 545] [INSPIRE].

[81] T. Becher and M. Neubert, Drell-Yan production at small $q_{T}$, transverse parton distributions and the collinear anomaly, Eur. Phys. J. C 71 (2011) 1665 [arXiv:1007.4005] [InSPIRE].

[82] S. Catani and L. Trentadue, Comment on QCD exponentiation at large $x$, Nucl. Phys. B 353 (1991) 183 [INSPIRE].

[83] V. Ahrens, A. Ferroglia, M. Neubert, B.D. Pecjak and L.-L. Yang, RG-improved single-particle inclusive cross sections and forward-backward asymmetry in $t \bar{t}$ production at hadron colliders, JHEP 09 (2011) 070 [arXiv:1103.0550] [INSPIRE].

[84] T. Becher and M.D. Schwartz, Direct photon production with effective field theory, JHEP 02 (2010) 040 [arXiv: 0911.0681] [INSPIRE].

[85] M.D. Schwartz, Resummation and NLO matching of event shapes with effective field theory, Phys. Rev. D 77 (2008) 014026 [arXiv:0709.2709] [InSPIRE].

[86] Y.-T. Chien and M.D. Schwartz, Resummation of heavy jet mass and comparison to LEP data, JHEP 08 (2010) 058 [arXiv: 1005.1644] [INSPIRE].

[87] P. Achard et al., Generalized event shape and energy flow studies in $e^{+} e^{-}$annihilation at $\sqrt{s}=91.2 \mathrm{GeV}-208.0 \mathrm{GeV}$, JHEP 10 (2011) 143 [INSPIRE].

[88] O. Tarasov, A. Vladimirov and A.Y. Zharkov, The Gell-Mann-Low function of QCD in the three loop approximation, Phys. Lett. B 93 (1980) 429 [INSPIRE].

[89] S. Larin and J. Vermaseren, The three loop QCD $\beta$-function and anomalous dimensions, Phys. Lett. B 303 (1993) 334 [hep-ph/9302208] [INSPIRE]. 